\title{
Reducing the Overhead of MPC over a Large Population
}

\author{
A. Choudhury ${ }^{1}$, A. Patra ${ }^{2}$, and N. P. Smart ${ }^{3}$ \\ 1 IIIT Bangalore, India. \\ 2 Dept. of Computer Science \& Automation, IISc Bangalore, India. \\ 3 Dept. of Computer Science, Uni. Bristol, United Kingdom. \\ partho31@gmail.com, arpita@csa.iisc.ernet.in, nigel@cs.bris.ac.uk.
}

\begin{abstract}
We present a secure honest majority MPC protocol, against a static adversary, which aims to reduce the communication cost in the situation where there are a large number of parties and the number of adversarially controlled parties is relatively small. Our goal is to reduce the usage of point-to-point channels among the parties, thus enabling them to run multiple different protocol executions. Our protocol has highly efficient theoretical communication cost when compared with other protocols in the literature; specifically the circuit-dependent communication cost, for circuits of suitably large depth, is $\mathcal{O}\left(|c k t| \kappa^{7}\right)$, for security parameter $\kappa$ and circuit size $\mid$ ckt $\mid$. Our protocol finds application in cloud computing scenario, where the fraction of corrupted parties is relatively small. By minimizing the usage of point-to-point channels, our protocol can enable a cloud service provider to run multiple MPC protocols.
\end{abstract}

\section{Introduction}

Threshold secure multi-party computation (MPC) is a fundamental problem in secure distributed computing. It allows a set of $n$ mutually distrusting parties with private inputs to "securely" compute any publicly known function of their private inputs, even in the presence of a centralized adversary who can control any $t$ out of the $n$ parties and force them to behave in any arbitrary manner. Now consider a situation, where $n$ is very large, say $n \geq 1000$ and the proportion of corrupted parties (namely the ratio $t / n$ ) is relatively small, say 5 percent. In such a scenario, involving all the $n$ parties to perform an MPC calculation is wasteful, as typical (secret-sharing based) MPC protocols require all parties to simultaneously transmit data to all other parties. However, restricting to a small subset of parties may lead to security problems. In this paper we consider the above scenario and show how one can obtain a communication efficient, robust MPC protocol which is actively secure against a computationally bounded static adversary. In particular we present a protocol in which the main computation is performed by a "smallish" subset of the parties, with the whole set of parties used occasionally so as to "checkpoint" the computation. By not utilizing the entire set of parties all the time enables them to run many MPC calculations at once. The main result we obtain in the paper is as follows:

Main Result (Informal): Let $\epsilon=\frac{t}{n}$ with $0 \leq \epsilon<1 / 2$ and let the $t$ corrupted parties be under the control of a computationally bounded static adversary. Then for a security parameter $\kappa$ (for example $\kappa=80$ or $\kappa=128$ ), there exists an MPC protocol with the following circuit-dependent communication complexity ${ }^{4}$ to evaluate an arithmetic circuit ckt: (a). $\mathcal{O}\left(|c k t| \cdot \kappa^{7}\right)$ for ckt with depth $\omega(t)$. (b). $\mathcal{O}\left(|\mathrm{ckt}| \cdot \kappa^{4}\right)$ for ckt with $d=\omega(t)$ and $w=\omega\left(\kappa^{3}\right)$ (i.e. $|\mathrm{ckt}|=\omega\left(\kappa^{3} t\right)$ ).

Protocol Overview: We make use of two secret-sharing schemes. A secret-sharing scheme [.] which is an actively-secure variant of the Shamir secret-sharing scheme [28] with threshold $t$. This first secret-sharing

\footnotetext{
${ }^{4}$ The communication complexity of an MPC protocol has two parts: a circuit-dependent part, dependent on the circuit size and a circuit-independent part. The focus is on the circuit-dependent communication, based on the assumption that the circuit is large enough so that the terms independent of the circuit-size can be ignored; see for example $[11,4,12,5]$.
} 
scheme is used to share values amongst all of the $n$ parties. The second secret-sharing scheme $\langle\cdot\rangle$ is an actively-secure variant of an additive secret-sharing scheme, amongst a well-defined subset $\mathcal{C}$ of the parties.

Assuming the inputs to the protocol are [.] shared amongst the parties at the start of the protocol, we proceed as follows. We first divide ckt into $L$ levels, where each level consists of a sub-circuit. The computation now proceeds in $L$ phases; we describe phase $i$. At the start of phase $i$ we have that all $n$ parties hold [.] sharings of the inputs to level $i$. The $n$ parties then select (at random) a committee $\mathcal{C}$ of size $\mathfrak{c}$. If $\mathfrak{c}$ is such that $\epsilon^{\mathfrak{c}}<2^{-\kappa}$ then statistically the committee $\mathcal{C}$ will contain at least one honest party, as the inequality implies that the probability that the committee contains no honest party is negligibly small. The $n$ parties then engage in a "conversion" protocol so that the input values to level $i$ are now $\langle\cdot\rangle$ shared amongst the committee. The committee $\mathcal{C}$ then engages in an actively-secure dishonest majority ${ }^{5}$ MPC protocol to evaluate the sub-circuit at level $i$. If no abort occurs during the evaluation of the $i$ th sub-circuit then the parties engage in another "conversion" protocol so that the output values of the sub-circuit are converted from a $\langle\cdot\rangle$ sharing amongst members in $\mathcal{C}$ to a $[\cdot]$ sharing amongst all $n$ parties. This step amounts to check-pointing data. This ensures that the inputs to all the subsequent sub-circuits are saved in the form of [.] sharing which guarantees recoverability as long as $0 \leq \epsilon<\frac{1}{2}$. So the check-pointing prevents from re-evaluating the entire circuit from scratch after every abort of the dishonest-majority MPC protocol.

If however an abort occurs while evaluating the $i$ th sub-circuit then we determine a pair of parties from the committee $\mathcal{C}$, one of whom is guaranteed to be corrupted and eliminate the pair from the set of active parties, and re-evaluate the sub-circuit again. In fact, cheating can also occur in the $\langle\cdot\rangle \leftrightarrow[\cdot]$ conversions and we need to deal with these as well. Thus if errors are detected we need to repeat the evaluation of the sub-circuit at level $i$. Since there are at most $t$ bad parties, the total amount of backtracking (i.e. evaluating a sub-circuit already computed) that needs to be done is bounded by $t$. For large $n$ and small $t$ this provides an asymptotically efficient protocol.

The main technical difficulty is in providing actively-secure conversions between the two secret-sharing schemes, and providing a suitable party-elimination strategy for the dishonest majority MPC protocol. The party-elimination strategy we employ follows from standard techniques, as long as we can identify the pair of parties. This requirement, of a dishonest-majority MPC protocol which enables identification of cheaters, without sacrificing privacy, leads us to the utilization of the protocol in [12]. This results in us needing to use double-trapdoor homomorphic commitments as a basic building block. To ensure greater asymptotic efficiency we apply two techniques: (a). the check-pointing is done among a set of parties that assures honest majority with overwhelming probability (b). the packing technique from [20] to our Shamir based secret sharing.

To obtain an efficient protocol one needs to select $L$; if $L$ is too small then the sub-circuits are large and so the cost of returning to a prior checkpoint will also be large. If however $L$ is too large then we will need to checkpoint a lot, and hence involve all $n$ parties in the computation at a lot of stages (and thus requiring all $n$ parties to be communicating/computing). The optimal value of $L$ for our protocol turns out to be $t$.

Related Work: The circuit-dependent communication complexity of the traditional MPC protocols in the honest-majority setting is $\mathcal{O}(|\mathrm{ckt}|$. Poly $(n, \kappa))$; this informally stems from the fact in these protocols we require all the $n$ parties to communicate with each other for evaluating each gate of the circuit. Assuming $0 \leq \epsilon<1 / 2$, [11] presents a computationally secure MPC protocol with communication complexity $\mathcal{O}(|\mathrm{ckt}| \cdot \operatorname{Poly}(\kappa, \log n, \log |\mathrm{ckt}|))$. The efficiency comes from the ability to pack and share several values simultaneously which in turn allow parallel evaluation of "several" gates simultaneously in a single round

\footnotetext{
${ }^{5}$ In the dishonest-majority setting, the adversary may corrupt all but one parties. An MPC protocol in this setting aborts if a corrupted party misbehaves.
} 
of communication. However, the protocol still requires communications between all the parties during each round of communication. Our protocol reduces the need for the parties to be communicating with all others at all stages in the protocol; moreover, asymptotically for large $n$ it provides a better communication complexity over [11] (as there is no dependence on $n$ ), for circuits of suitably large depth as stated earlier. However, the protocol of [11] is secure against a more powerful adaptive adversary.

In the literature, another line of investigation has been carried out in $[6,10,14,15]$ to beat the $\mathcal{O}(|c k t|$. Poly $(n, \kappa))$ communication complexity bound of traditional MPC protocols, against a static adversary. The main idea behind all these works is similar to ours, which is to involve "small committees" of parties for evaluating each gate of the circuit, rather than involving all the $n$ parties. The communication complexity of these protocols ${ }^{6}$ is of the order $\mathcal{O}(|c k t| \cdot \operatorname{Poly}(\log n, \kappa))$. Technically our protocol is different from these protocols in the following ways: (a). The committees in $[6,10,14,15]$ are of size Poly $(\log n)$, which ensures that with high probability the selected committees have honest majority. As a result, these protocols run any existing honest-majority MPC protocol among these small committees of Poly $(\log n)$ size, which prevents the need to check-point the computation (as there will be no aborts). On the other hand, we only require committees with at least one honest party and our committee size is independent of $n$, thus providing better communication complexity. Indeed, asymptotically for large $n$, our protocol provides a better communication complexity over $[6,10,14,15]$ (as there is no dependence on $n$ ), for circuits of suitably large depth. (b). Our protocol provides a better fault-tolerance. Specifically, [14, 10,6] requires $\epsilon<1 / 3$ and [15] requires $\epsilon<1 / 8$; on the other hand we require $\epsilon<1 / 2$.

We stress that the committee selection protocol in $[6,10,14,15]$ is unconditionally secure and in the full-information model, where the corrupted parties can see all the messages communicated between the honest parties. On the other hand our implementation of the committee selection protocol is computationally secure. The committee election protocol in $[6,10,14,15]$ is inherited from [17]. The committee selection protocol in these protocols are rather involved and not based on simply randomly selecting a subset of parties, possibly due to the challenges posed in the full information model with unconditional security; this causes their committee size to be logarithmic in $n$. However, if one is willing to relax at least one of the above two features (i.e. full information model and unconditional security), then it may be possible to select committees with honest majority in a simple way by randomly selecting committees, where the committee size may be independent of $n$. However investigating the same is out of the scope of this paper.

Finally we note that the idea of using small committees has been used earlier in the literature for various distributed computing tasks, such as the leader election [23,26], Byzantine agreement [24,25] and distributed key-generation [9].

On the Choice of $\epsilon$ : We select committees of size $\mathfrak{c}$ satisfying $\epsilon^{\mathfrak{c}}<2^{-\kappa}$. This implies that the selected committee has at least one honest participant with overwhelming probability. We note that it is possible to randomly select committees of "larger" size so that with overwhelming probability the selected committee will have honest majority. We label the protocol which samples a committee with honest majority and then runs an computationally secure honest majority MPC protocol (where we need not have to worry about aborts) as the "naive protocol". The naive protocol will have communication complexity $\mathcal{O}(|\mathrm{ckt}| \cdot \operatorname{Poly}(\kappa))$.

For "very small" values of $\epsilon$, the committee size for the naive protocol is comparable to the committee size in our protocol. We demonstrate this with an example, with $n=1000$ and security level $\kappa=80$ : The committee size we require to ensure both a single honest party in the committee and a committee with honest majority, with overwhelming probability of $\left(1-2^{-80}\right)$ for various choices of $\epsilon$, is given in the following table:

\footnotetext{
${ }^{6}$ Note, the protocol of [6] involves FHE to further achieve a communication complexity of $\mathcal{O}(\operatorname{Poly}(\log n))$.
} 


\begin{tabular}{|c|c|c|}
\hline$\epsilon$ & $\mathfrak{c}$ to obtain at least one honest party & $\mathfrak{c}$ to obtain honest majority \\
\hline $1 / 3$ & 48 & 448 \\
\hline $1 / 4$ & 39 & 250 \\
\hline $1 / 10$ & 23 & 84 \\
\hline $1 / 100$ & 11 & 20 \\
\hline
\end{tabular}

From the table it is clear that when $\epsilon$ is closer to $1 / 2$, the difference in the committee size to obtain at least one honest party and to obtain honest majority is large. As a result, selecting committees with honest majority can be prohibitively expensive, thus our selection of small committees with dishonest majority provides significant improvements.

To see intuitively why our protocol selects smaller committees, consider the case when the security parameter $\kappa$ tends to infinity: Our protocol will require a committee of size roughly $\epsilon \cdot n+1$, whereas the naive protocol will require a committee of size roughly $2 \cdot \epsilon \cdot n+1$. Thus the naive method will use a committee size of roughly twice that of our method. Hence, if small committees are what is required then our method improves on the naive method.

For fixed $\epsilon$ and increasing $n$, we can apply the binomial approximation to the hypergeometric distribution, and see that our protocol will require a committee of size $c \approx \kappa / \log _{2}\left(\frac{1}{\epsilon}\right)$. To estimate the committee size for the naive protocol we use the cumulative distribution function for the binomial distribution, $F(b ; c, \epsilon)$, which gives the probability that we select at least $b$ corrupt parties in a committee of size $c$ given the probability of a corrupt party being fixed at $\epsilon$. To obtain an honest majority with probability less than $2^{-\kappa}$ we require $F(c / 2 ; c, \epsilon) \approx 2^{-\kappa}$. By estimating $F(c / 2 ; c, \epsilon)$ via Hoeffding's inequality we obtain

$$
\exp \left(-2 \cdot \frac{(c \cdot \epsilon-c / 2)^{2}}{c}\right) \approx 2^{-\kappa}
$$

which implies

$$
\kappa \approx\left(\frac{c \cdot(2 \cdot \epsilon-1)^{2}}{2}\right) / \log _{e} 2
$$

Solving for $c$ gives us

$$
c \approx \frac{2 \cdot \kappa \cdot \log _{e} 2}{(2 \cdot \epsilon-1)^{2}}
$$

Thus for fixed $\epsilon$ and large $n$ the number of parties in a committee is $O(\kappa)$ for both our protocol, and the naive protocol. Thus the communication complexity of our protocol and the naive protocol is asymptotically the same. But, since the committees in our protocol are always smaller than those in the naive protocol, we will obtain an advantage when the ratio of the different committee size is large, i.e. when $\epsilon$ is larger.

The the ratio between the committee size in the naive protocol and that of our protocol (assuming we are in a range when Hoeffding's inequality provides a good approximation) is roughly

$$
\frac{-2 \cdot \log _{e} 2 \cdot \log _{2} \epsilon}{(2 \cdot \epsilon-1)^{2}}
$$

So for large $n$ the ratio between the committee sizes of the two protocols depends on $\epsilon$ alone (and is independent of $\kappa$ ). By way of example this ratio is approximately equal to 159 when $\epsilon=0.45,19$ when $\epsilon=1 / 3$, 7 when $\epsilon=1 / 10$ and 9.6 when $\epsilon=1 / 100$; although the approximation via Hoeffding's inequality only really applies for $\epsilon$ close to $1 / 2$.

This implies that for values of $\epsilon$ close to $1 / 2$ our protocol will be an improvement on the naive protocol. However, the naive method does not have the extra cost of check-pointing which our method does; thus at some point the naive protocol will be more efficient. Thus our protocol is perhaps more interesting, when $\epsilon$ is not too small, say in the range of $[1 / 100,1 / 2]$. 
Possible Application of Our Protocol for Cloud-Computing. Consider the situation of an organization performing a multi-party computation on a cloud infrastructure, which involves a large number of machines, with the number of corrupted parties possibly high, but not exceeding one half of the parties, (which is exactly the situation considered in our MPC protocol). Using our MPC protocol, the whole computation can be then carried out by a small subset of machines, with the whole cloud infrastructure being used only for check-pointing the computation. By not utilizing the whole cloud infrastructure all the time, we enable the cloud provider to serve multiple MPC requests.

Our protocol is not adaptively secure. In fact, vulnerability to adaptive adversary is inherent to most of the committee-based protocols for several distributed computing tasks such as Leader Election [23, 26], Byzantine Agreement [25, 24], Distributed Key-generation [9] and MPC in [14, 10]. Furthermore, We feel that adaptive security is not required in the cloud scenario. Any external attacker to the cloud data centre will have a problem determining which computers are being used in the committee, and an even greater problem in compromising them adaptively. The main threat model in such a situation is via co-tenants (other users processes) to be resident on the same physical machine. Since the precise machine upon which a cloud tenant sits is (essentially) randomly assigned, it is hard for a co-tenant adversary to mount a cross-Virtual Machine attack on a specific machine unless they are randomly assigned this machine by the cloud. Note, that co-tenants have more adversarial power than a completely external attacker. A more correct security model would be to have a form of adaptive security in which attackers pro-actively move from one machine to another, but in a random fashion. We leave analysing this complex situation to a future work.

\section{Model, Notation and Preliminaries}

We denote by $\mathcal{P}=\left\{P_{1}, \ldots, P_{n}\right\}$ the set of $n$ parties who are connected by pair-wise private and authentic channels. We assume that there exists a PPT static adversary $\mathcal{A}$, who can maliciously corrupt any $t$ parties from $\mathcal{P}$ at the beginning of the execution of a protocol, where $t=n \cdot \epsilon$ and $0 \leq \epsilon<\frac{1}{2}$. There exists a publicly known randomized function $f: \mathbb{F}_{p}^{n} \rightarrow \mathbb{F}_{p}$, expressed as a publicly known arithmetic circuit ckt over the field $\mathbb{F}_{p}$ of prime order $p$ (including random gates to enable the evaluation of randomized functions), with party $P_{i}$ having a private input $x^{(i)} \in \mathbb{F}_{p}$ for the computation. We let $d$ and $w$ to denote the depth and (average) width of ckt respectively. The finite field $\mathbb{F}_{p}$ is assumed to be such that $p$ is a prime, with $p>\max \left\{n, 2^{\kappa}\right\}$, where $\kappa$ is the computational security parameter. Apart from $\kappa$, we also have an additional statistical security parameter $s$ and the security offered by $s$ (which is generally much smaller than $\kappa$ ) does not depend on the computational power of the adversary.

The security of our protocol(s) will be proved in the universal composability (UC) model. The UC framework allows for defining the security properties of cryptographic tasks so that security is maintained under general composition with an unbounded number of instances of arbitrary protocols running concurrently. In the framework, the security requirements of a given task are captured by specifying an ideal functionality run by a "trusted party" that obtains the inputs of the parties and provides them with the desired outputs. Informally, a protocol securely carries out a given task if running the protocol in the presence of a real-world adversary amounts to "emulating" the desired functionality. For more details, see Appendix A.

We do not assume a physical broadcast channel. Although our protocol uses an ideal broadcast functionality $\mathcal{F}_{\mathrm{BC}}$ (Fig. 3), that allows a sender Sen $\in \mathcal{P}$ to reliably broadcast a message to a group of parties $\mathcal{X} \subseteq \mathcal{P}$, the functionality can be instantiated using point-to-point channels; see Appendix B.2 for details.

The communication complexity of our protocols has two parts: the communication done over the pointto-point channels and the broadcast communication. The later is captured by $\mathcal{B C}(\ell,|\mathcal{X}|)$ to denote that in total, $\mathcal{O}(\ell)$ bits is broadcasted in the associated protocol to a set of parties of size $|\mathcal{X}|$. For details about the instantiation of $\mathcal{F}_{\mathrm{BC}}$, see Appendix B. 
Two different types of secret-sharing are employed in our protocols. The secret-sharings are inherently defined to include "verification information" of the individual shares in the form of publicly known commitments. We use a variant of the Pedersen homomorphic commitment scheme [27]. In our protocol, we require UC-secure commitments to ensure that a committer must know its committed value and just cannot manipulate a commitment produced by other committers to violate what we call "input independence". It has been shown in [8] that a UC secure commitment scheme is impossible to achieve without setup assumptions. The standard method to implement UC-secure commitments is in the Common Reference String (CRS) model where it is assumed that the parties are provided with a CRS that is set up by a "trusted third party" (TTP). We follow [12], where the authors show how to build a multiparty UC-secure homomorphic commitment scheme (where multiple parties can act as committer) based on any double-trapdoor homomorphic commitment scheme.

Definition 1 (Double-trapdoor Homomorphic Commitment for $\left.\mathbb{F}_{p}[12]\right)$. It is a collection of five PPT algorithms (Gen, Comm, Open, Equivocate, TDExtract, $\odot$ ):

- $\operatorname{Gen}\left(1^{\kappa}\right) \rightarrow\left(\mathrm{ck}, \tau_{0}, \tau_{1}\right):$ the generation algorithm outputs a commitment key ck, along with trapdoors $\tau_{0}$ and $\tau_{1}$.

- $\operatorname{Comm}_{\mathrm{ck}}\left(x ; r_{0}, r_{1}\right) \rightarrow \mathbf{C}_{x, r_{0}, r_{1}}$ : the commitment algorithm takes a message $x \in \mathbb{F}_{p}$ and randomness $r_{0}, r_{1}$ from the commitment randomness space $\mathcal{R}^{7}$ and outputs a commitment $\mathbf{C}_{x ; r_{0}, r_{1}}$ of $x$ under the randomness $r_{0}, r_{1}$.

- Open $_{\mathrm{ck}}\left(\mathbf{C},\left(x ; r_{0}, r_{1}\right)\right) \rightarrow\{0,1\}$ : the opening algorithm takes a commitment $\mathbf{C}$, along with a message/randomness triplet $\left(x, r_{0}, r_{1}\right)$ and outputs 1 if $\mathbf{C}=\operatorname{Comm}_{\mathrm{ck}}\left(x ; r_{0}, r_{1}\right)$, else 0 .

- Equivocate $\left(\mathbf{C}_{x, r_{0}, r_{1}}, x, r_{0}, r_{1}, \bar{x}, \tau_{i}\right) \rightarrow\left(\bar{r}_{0}, \bar{r}_{1}\right) \in \mathcal{R}:$ using one of the trapdoors $\tau_{i}$ with $i \in\{0,1\}$, the equivocation algorithm can open a commitment $\mathbf{C}_{x, r_{0}, r_{1}}$ with any message $\bar{x} \neq x$ with randomness $\bar{r}_{0}$ and $\bar{r}_{1}$ where $r_{1-i}=\bar{r}_{1-i}$.

- TDExtract $\left(\mathbf{C}, x, r_{0}, r_{1}, \bar{x}, \bar{r}_{0}, \bar{r}_{1}, \tau_{i}\right) \rightarrow \tau_{1-i}$ : using one of the trapdoors $\tau_{i}$ with $i \in\{0,1\}$ and two different sets of message/randomness triplet for the same commitment, namely $x, r_{0}, r_{1}$ and $\bar{x}, \bar{r}_{0}, \bar{r}_{1}$, the trapdoor extraction algorithm can find the other trapdoor $\tau_{1-i}$ if $r_{1-i} \neq \bar{r}_{1-i}$.

The commitments are homomorphic meaning that $\operatorname{Comm}\left(x ; r_{0}, r_{1}\right) \odot \operatorname{Comm}\left(y ; s_{0}, s_{1}\right)=\operatorname{Comm}(x+$ $\left.y ; r_{0}+s_{0}, r_{1}+s_{1}\right)$ and $\operatorname{Comm}\left(x ; r_{0}, r_{1}\right)^{c}=\operatorname{Comm}\left(c \cdot x ; c \cdot r_{0}, c \cdot r_{1}\right)$ for any publicly known constant $c$.

We require the following properties to be satisfied:

- Trapdoor Security: There exists no PPT algorithm $A$ such that $A\left(1^{\kappa}, \mathrm{ck}, \tau_{i}\right) \rightarrow \tau_{1-i}$, for $i \in\{0,1\}$.

- Computational Binding: There exists no PPT algorithm $A$ with $A\left(1^{\kappa}, \mathrm{ck}\right) \rightarrow\left(x, r_{0}, r_{1}, \bar{x}, \bar{r}_{0}, \bar{r}_{1}\right)$ and $\left(x, r_{0}, r_{1}\right) \neq\left(\bar{x}, \bar{r}_{0}, \bar{r}_{1}\right)$, but $\operatorname{Comm}_{\mathrm{ck}}\left(x ; r_{0}, r_{1}\right)=\operatorname{Comm}_{\mathrm{ck}}\left(\bar{x} ; \bar{r}_{0}, \bar{r}_{1}\right)$.

- Statistical Hiding: $\forall x, \bar{x} \in \mathbb{F}_{p}$ and $r_{0}, r_{1} \in \mathcal{R}$, let $\left(\bar{r}_{0}, \bar{r}_{1}\right)=$ Equivocate $\left(\mathbf{C}_{x, r_{0}, r_{1}}, x, r_{0}, r_{1}, \bar{x}, \tau_{i}\right)$, with $i \in\{0,1\}$. Then $\operatorname{Comm}_{\mathrm{ck}}\left(x ; r_{0}, r_{1}\right)=\operatorname{Comm}_{\mathrm{ck}}\left(\bar{x} ; \bar{r}_{0}, \bar{r}_{1}\right)=\mathbf{C}_{x, r_{0}, r_{1}} ;$ moreover the distribution of $\left(r_{0}, r_{1}\right)$ and $\left(\bar{r}_{0}, \bar{r}_{1}\right)$ are statistically close.

We will use the following instantiation of a double-trapdoor homomorphic commitment scheme which is a variant of the standard Pedersen commitment scheme over a group $\mathbb{G}$ in which discrete logarithms are hard [12]. The message space is $\mathbb{F}_{p}$ and the randomness space is $\mathcal{R}=\mathbb{F}_{p}^{2}$.

- Gen $\left(1^{\kappa}\right) \rightarrow\left(\left(\mathbb{G}, p, g, h_{0}, h_{1}\right), \tau_{0}, \tau_{1}\right)$, where ck $=\left(\mathbb{G}, p, g, h_{0}, h_{1}\right)$ such that $g, h_{0}, h_{1}$ are generators of the group $\mathbb{G}$ of prime order $p$ and $g^{\tau_{i}}=h_{i}$ for $i \in\{0,1\}$.

\footnotetext{
${ }^{7}$ For the ease of presentation, we assume $\mathcal{R}$ to be an additive group.
} 
- $\operatorname{Comm}_{\mathrm{ck}}\left(x ; r_{0}, r_{1}\right) \rightarrow g^{x} h_{0}^{r_{0}} h_{1}^{r_{1}}=\mathbf{C}_{x, r_{0}, r_{1}}$, with $x, r_{0}, r_{1} \in \mathbb{F}_{p}$.

- Open ${ }_{\mathrm{ck}}\left(\mathbf{C},\left(x, r_{0}, r_{1}\right)\right) \rightarrow 1$, if $\mathbf{C}=g^{x} h_{0}^{r_{0}} h_{1}^{r_{1}}$, else Open $\mathrm{ck}\left(\mathbf{C},\left(x, r_{0}, r_{1}\right)\right) \rightarrow 0$.

- Equivocate $\left(\mathbf{C}_{x, r_{0}, r_{1}}, x, r_{0}, r_{1}, \bar{x}, \tau_{i}\right) \rightarrow\left(\bar{r}_{0}, \bar{r}_{1}\right)$ where $\bar{r}_{1-i}=r_{1-i}$ and $\bar{r}_{i}=\tau_{i}^{-1}(x-\bar{x})+r_{i}$.

- TDExtract $\left(\mathbf{C}, x, r_{0}, r_{1}, \bar{x}, \bar{r}_{0}, \bar{r}_{1}, \tau_{i}\right) \rightarrow \tau_{1-i}$, where if $\bar{r}_{1-i} \neq r_{1-i}$, then

$$
\tau_{1-i}=\frac{\bar{x}-x+\tau_{i}\left(\bar{r}_{i}-r_{i}\right)}{r_{1-i}-\bar{r}_{1-i}} .
$$

- The homomorphic operation $\odot$ is just the group operation i.e.

$$
\begin{aligned}
\operatorname{Comm}\left(x ; r_{0}, r_{1}\right) \odot \operatorname{Comm}\left(\bar{x} ; \bar{r}_{0}, \bar{r}_{1}\right) & =g^{x} h_{0}^{r_{0}} h_{1}^{r_{1}} \cdot g^{\bar{x}} h_{0}^{\bar{r}_{0}} h_{1}^{\bar{r}_{1}} \\
& =g^{x+\bar{x}} \cdot h_{0}^{r_{0}+\bar{r}_{0}} \cdot h_{1}^{r_{1}+\bar{r}_{1}} \\
& =\operatorname{Comm}\left(x+\bar{x} ; r_{0}+\bar{r}_{0}, r_{1}+\bar{r}_{1}\right) .
\end{aligned}
$$

We can now define the various types of secret-shared data used in our protocols. Let $\alpha_{1}, \ldots, \alpha_{n} \in \mathbb{F}_{p}$ be $n$ publicly known non-zero, distinct values, where $\alpha_{i}$ is associated with $P_{i}$ as the evaluation point. The [.] sharing is the standard Shamir-sharing [28], where the secret value will be shared among the set of parties $\mathcal{P}$ with threshold $t$. Additionally, a commitment of each individual share will be available publicly, with the corresponding share-holder possessing the randomness of the commitment.

Definition 2 (The [.] Sharing). Let $s \in \mathbb{F}_{p}$; then $s$ is said to be [.]-shared among $\mathcal{P}$ if there exist polynomials, say $f(\cdot), g(\cdot)$ and $h(\cdot)$, of degree at most $t$, with $f(0)=s$ and every (honest) party $P_{i} \in \mathcal{P}$ holds a share $f_{i}=f\left(\alpha_{i}\right)$ of $s$, along with opening information $g_{i}=g\left(\alpha_{i}\right)$ and $h_{i}=h\left(\alpha_{i}\right)$ for the commitment $\mathbf{C}_{f_{i}, g_{i}, h_{i}}=\operatorname{Comm}_{\mathrm{ck}}\left(f_{i} ; g_{i}, h_{i}\right)$. The information available to party $P_{i} \in \mathcal{P}$ as part of the [.]-sharing of $s$ is denoted by $[s]_{i}=\left(f_{i}, g_{i}, h_{i},\left\{\mathbf{C}_{f_{j}, g_{j}, h_{j}}\right\}_{P_{j} \in \mathcal{P}}\right)$. All parties will also have the access to ck. Moreover, the collection of $[s]_{i}$ 's, corresponding to $P_{i} \in \mathcal{P}$ is denoted by $[s]$.

The second type of secret-sharing (which is a variation of additive sharing), is used to perform computation via a dishonest majority MPC protocol amongst our committees.

Definition 3 (The $\langle\cdot\rangle$ Sharing). A value $s \in \mathbb{F}_{p}$ is said to be $\langle\cdot\rangle$-shared among a set of parties $\mathcal{X} \subseteq \mathcal{P}$, if every (honest) party $P_{i} \in \mathcal{X}$ holds a share $s_{i}$ of $s$ along with the opening information $u_{i}, v_{i}$ for the commitment $\mathbf{C}_{s_{i}, u_{i}, u_{i}}=\operatorname{Comm}_{\mathrm{ck}}\left(s_{i} ; u_{i}, v_{i}\right)$, such that $\sum_{P_{i} \in \mathcal{X}} s_{i}=s$. The information available to party $P_{i} \in \mathcal{X}$ as part of the $\langle\cdot\rangle$-sharing of $s$ is denoted by $\langle s\rangle_{i}=\left(s_{i}, u_{i}, v_{i},\left\{\mathbf{C}_{s_{j}, u_{j}, v_{j}}\right\}_{P_{j} \in \mathcal{X}}\right)$. All parties will also have access to $\mathrm{ck}$. The collection of $\langle s\rangle_{i}$ 's corresponding to $P_{i} \in \mathcal{X}$ is denoted by $\langle s\rangle_{\mathcal{X}}$.

It is easy to see that both types of secret-sharing are linear. For example, for the $\langle\cdot\rangle$ sharing, given $\left\langle s^{(1)}\right\rangle \mathcal{X}, \ldots$, $\left\langle s^{(\ell)}\right\rangle_{\mathcal{X}}$ and publicly known constants $c_{1}, \ldots, c_{\ell}$, the parties in $\mathcal{X}$ can locally compute their information corresponding to $\left\langle c_{1} \cdot s^{(1)}+\ldots+c_{\ell} \cdot s^{(\ell)}\right\rangle \mathcal{X}$. This follows from the homomorphic property of the underlying commitment scheme and the linearity of the secret-sharing scheme. This means that the parties in $\mathcal{X}$ can locally compute $\left\langle c_{1} \cdot s^{(1)}+\ldots+c_{\ell} \cdot s^{(\ell)}\right\rangle_{\mathcal{X}}$ from $\left\langle s^{(1)}\right\rangle_{\mathcal{X}}, \ldots,\left\langle s^{\ell}\right\rangle_{\mathcal{X}}$, since each party $P_{i}$ in $\mathcal{X}$ can locally compute $\left\langle c_{1} \cdot s^{(1)}+\ldots+c_{\ell} \cdot s^{(\ell)}\right\rangle_{i}$ from $\left\langle s^{(1)}\right\rangle_{i}, \ldots,\left\langle s^{\ell}\right\rangle_{i}$.

\section{Main Protocol}

We now present an MPC protocol implementing the standard honest-majority (meaning $\epsilon<1 / 2$ ) MPC functionality $\mathcal{F}_{f}$ presented in Figure 1 which computes the function $f$.

We now present the underlying idea of our protocol (outlined earlier in the introduction). The protocol is set in a variant of the player-elimination framework from [4]. During the computation either pairs of parties, 


\section{Functionality $\mathcal{F}_{f}$}

$\mathcal{F}_{f}$ interacts with the parties in $\mathcal{P}$ and the adversary $\mathcal{S}$ and is parametrized by an $n$-input function $f: \mathbb{F}_{p}^{n} \rightarrow \mathbb{F}_{p}$.

- Upon receiving $\left(\operatorname{sid}, i, x^{(i)}\right)$ from every $P_{i} \in \mathcal{P}$ where $x^{(i)} \in \mathbb{F}_{p}$, the functionality computes $y=f\left(x^{(1)}, \ldots, x^{(n)}\right)$, sends (sid, $y$ ) to all the parties and the adversary $\mathcal{S}$ and halts.

Fig. 1. The Ideal Functionality for Computing a Given Function $f$

each containing at least one actively corrupted party, or singletons of corrupted parties, are identified due to some adversarial behavior of the corrupted parties. These pairs, or singletons, are then eliminated from the set of eligible parties. To understand how we deal with the active corruptions, we need to define a dynamic set $\mathcal{L} \subseteq \mathcal{P}$ of size $\mathfrak{n}$, which will define the current set of eligible parties in our protocol, and a threshold $\mathfrak{t}$ which defines the maximum number of corrupted parties in $\mathcal{L}$. Initially $\mathcal{L}$ is set to be equal to $\mathcal{P}$ (hence $\mathfrak{n}=n$ ) and $\mathfrak{t}$ is set to $t$. We then divide the circuit ckt (representing $f$ ) to be evaluated into $L$ levels, where each level consists of a sub-circuit of depth $d / L$; without loss of generality, we assume $d$ to be a multiple of $L$. We denote the $i$ th sub-circuit as $\mathrm{ckt}_{i}$. At the beginning of the protocol, all the parties in $\mathcal{P}$ verifiably $[\cdot]$-share their inputs for the circuit ckt.

For evaluating a sub-circuit $\mathrm{ckt}_{l}$, instead of involving all the parties in $\mathcal{L}$, we rather involve a small and random committee $\mathcal{C} \subset \mathcal{L}$ of parties of size $\mathfrak{c}$, where $\mathfrak{c}$ is the minimum value satisfying the constraint that $\epsilon^{\mathfrak{c}} \leq 2^{-\kappa}$; recall $\epsilon=t / n$. During the course of evaluating the sub-circuit, if any inconsistency is reported, then the (honest) parties in $\mathcal{P}$ will identify either a single corrupted party or a pair of parties from $\mathcal{L}$ where the pair contains at least one corrupted party. The identified party(ies) is(are) eliminated from $\mathcal{L}$ and the value of $\mathfrak{t}$ is decremented by one, followed by re-evaluation of $\mathrm{ckt}_{l}$ by choosing a new committee from the updated set $\mathcal{L}$. This is reminiscent of the player-elimination framework from [4], however the way we apply the player-elimination framework is different from the standard one. Specifically, in the player-elimination framework, the entire set of eligible parties $\mathcal{L}$ is involved in the computation and the player elimination is then performed over the entire $\mathcal{L}$, thus requiring huge communication. On the contrary, in our context, only a small set of parties $\mathcal{C}$ is involved in the computation, thus significantly reducing the communication complexity. It is easy to see that after a sequence of $t$ failed sub-circuit evaluations, $\mathcal{L}$ will be left with only honest parties and so each sub-circuit will be evaluated successfully from then onwards.

Note that the way we eliminate the parties, the fraction of corrupted parties in $\mathcal{L}$ after any un-successful attempt for sub-circuit evaluation, is upper bounded by the fraction of corrupted parties in $\mathcal{L}$ prior to the evaluation of the sub-circuit. Specifically, let $\epsilon_{\text {old }}=\mathfrak{t} / \mathfrak{n}$ be the fraction of corrupted parties in $\mathcal{L}$ prior to the evaluation of a sub-circuit $\mathrm{ckt}_{l}$ and let the evaluation fail, with either a single party or a pair of parties being eliminated from $\mathcal{L}$. Moreover, let $\epsilon_{\text {new }}$ be the fraction of corrupted parties in $\mathcal{L}$ after the elimination. Then for single elimination, we have $\epsilon_{\text {new }}=\frac{\mathfrak{t}-1}{\mathfrak{n}-1}$ and so $\epsilon_{\text {new }} \leq \epsilon_{\text {old }}$ if and only if $\mathfrak{n} \geq \mathfrak{t}$, which will always hold. On the other hand, for double elimination, we have $\epsilon_{\text {new }}=\frac{\mathfrak{t}-1}{\mathfrak{n}-2}$ and so $\epsilon_{\text {new }} \leq \epsilon_{\text {old }}$ if and only if $\mathfrak{n} \geq 2 \mathfrak{t}$, which will always hold.

Since a committee $\mathcal{C}$ (for evaluating a sub-circuit) is selected randomly, except with probability at most $\epsilon^{\mathfrak{c}}<2^{-\kappa}$, the selected committee contains at least one honest party and so the sub-circuit evaluation among $\mathcal{C}$ needs to be performed via a dishonest majority MPC protocol. We choose the MPC protocol of [12], since it can be modified to identify pairs of parties consisting of at least one corrupted party in the case of the failed evaluation, without violating the privacy of the honest parties. To use the protocol of [12] for subcircuit evaluation, we need the corresponding sub-circuit inputs (available to the parties in $\mathcal{P}$ in [.]-shared form) to be converted and available in $\langle\cdot\rangle$-shared form to the parties in $\mathcal{C}$ and so the parties in $\mathcal{P}$ do the same. After every successful evaluation of a sub-circuit, via the dishonest majority MPC protocol, the outputs of that sub-circuit (available in $\langle\cdot\rangle$-shared form to the parties in a committee) are converted and saved in the 
form of $[\cdot]$-sharing among all the parties in $\mathcal{P}$. As the set $\mathcal{P}$ has a honest majority, $[\cdot]$-sharing ensures robust reconstruction implying that the shared values are recoverable. Since the inputs to a sub-circuit come either from the outputs of previous sub-circuit evaluations or the original inputs, both of which are [.]-shared, a failed attempt for a sub-circuit evaluation does not require a re-evaluation of the entire circuit from scratch but requires a re-evaluation of that sub-circuit only.

\subsection{Supporting Functionalities}

We now present a number of ideal functionalities defining sub-components of our main protocol; see Appendix B for the UC-secure instantiations of these functionalities.

Basic Functionalities: The functionality $\mathcal{F}_{\mathrm{CRS}}$ for generating the common reference string (CRS) for our main MPC protocol is given in Figure 2. The functionality outputs the commitment key of a doubletrapdoor homomorphic commitment scheme, along with the encryption key of an IND-CCA secure encryption scheme (to be used later for UC-secure generation of completely random $\langle\cdot\rangle$-shared values as in [12]), see Appendix D. The functionality $\mathcal{F}_{\mathrm{BC}}$ for group broadcast is given in Figure 3 . This functionality broadcasts the message sent by a sender Sen $\in \mathcal{P}$ to all the parties in a sender specified set of parties $\mathcal{X} \subseteq \mathcal{P}$; in our context, the set $\mathcal{X}$ will always contain at least one honest party. The functionality $\mathcal{F}_{\text {CомmitтeE }}$ for a random committee selection is given in Figure 4. This functionality is parameterized by a value $\mathfrak{c}$, it selects a set $\mathcal{X}$ of $\mathfrak{c}$ parties at random from a specified set $\mathcal{Y}$ and outputs the selected set $\mathcal{X}$ to the parties in $\mathcal{P}$.

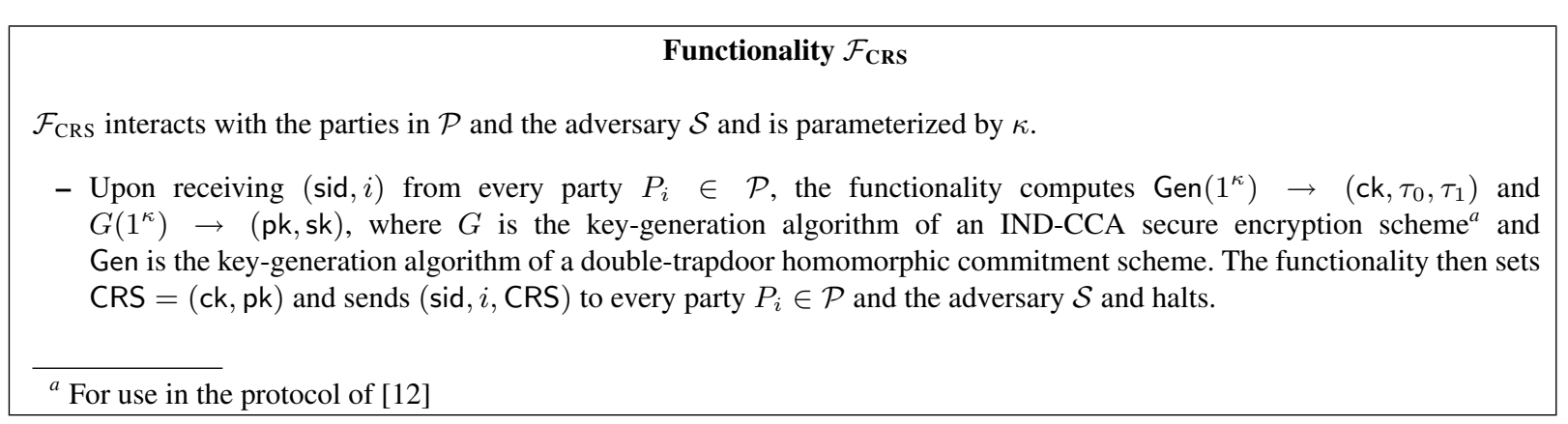

Fig. 2. The Ideal Functionality for Generating CRS

\section{Functionality $\mathcal{F}_{\mathbf{B C}}$}

$\mathcal{F}_{\mathrm{BC}}$ interacts with the parties in $\mathcal{P}$ and the adversary $\mathcal{S}$.

- Upon receiving (sid, Sen, $x, \mathcal{X}$ ) from the sender Sen $\in \mathcal{P}$ such that $\mathcal{X} \subseteq \mathcal{P}$, the functionality sends (sid, $j$, Sen, $x$ ) to every $P_{j} \in \mathcal{X}$ and to the adversary $\mathcal{S}$ and halts.

Fig. 3. The Ideal Functionality for Broadcast

Functionality Related to [·]-sharings: In Figure 5 we present the functionality $\mathcal{F}_{\text {Gen [.] }}$ which allows a dealer $\mathrm{D} \in \mathcal{P}$ to verifiably [.]-share an already committed secret among the parties in $\mathcal{P}$. The functionality is invoked when it receives three polynomials, say $f(\cdot), g(\cdot)$ and $h(\cdot)$ from the dealer $\mathrm{D}$ and a commitment, say $\mathbf{C}$, supposedly the commitment of $f(0)$ with randomness $g(0), h(0)$ (namely $\mathbf{C}_{f(0), g(0), h(0)}$ ), from the (majority of the) parties in $\mathcal{P}$. The functionality then hands $f_{i}=f\left(\alpha_{i}\right), g_{i}=g\left(\alpha_{i}\right), h_{i}=h\left(\alpha_{i}\right)$ and commitments $\left\{\mathbf{C}_{f_{j}, g_{j}, h_{j}}\right\}_{P_{j} \in \mathcal{P}}$ to $P_{i} \in \mathcal{P}$ after 'verifying' that (a): All the three polynomials are of degree at most 
Functionality $\mathcal{F}_{\text {Соммiтte }}$

$\mathcal{F}_{\text {СомміттеE }}$, parametrized by a constant $\mathfrak{c}$, interacts with the parties in $\mathcal{P}$ and the adversary $\mathcal{S}$.

- Upon receiving $(\operatorname{sid}, i, \mathcal{Y})$ from every $P_{i} \in \mathcal{P}$, the functionality selects $\mathfrak{c}$ parties at random from the set $\mathcal{Y}$ that is received from the majority of the parties and denotes the selected set as $\mathcal{X}$. The functionality then sends (sid, $i, \mathcal{X})$ to every $P_{i} \in \mathcal{P}$ and $\mathcal{S}$ and halts.

Fig. 4. The Ideal Functionality for Selecting a Random Committee of Given Size $\mathfrak{c}$

$t$ and (b): $\mathbf{C}=\operatorname{Comm}_{\mathrm{ck}}(f(0) ; g(0), h(0))$ i.e. the value (and the corresponding randomness) committed in $\mathbf{C}$ are embedded in the constant term of $f(\cdot), g(\cdot)$ and $h(\cdot)$ respectively. If either of the above two checks fail, then the functionality returns Failure to the parties indicating that $\mathrm{D}$ is corrupted.

In our MPC protocol where $\mathcal{F}_{\mathrm{GEN}[\cdot]}$ is called, the dealer will compute the commitment $\mathbf{C}$ as $\operatorname{Comm}_{\mathrm{ck}}(f(0)$; $g(0), h(0))$ and will broadcast it prior to making a call to $\mathcal{F}_{\mathrm{GEN}[\cdot]}$. It is easy to note that $\mathcal{F}_{\mathrm{GEN}[\cdot]}$ generates $[f(0)]$ if $\mathrm{D}$ is honest or well-behaved. If $\mathcal{F}_{\mathrm{GEN}[\cdot]}$ returns Failure, then $\mathrm{D}$ is indeed corrupted.

\section{Functionality $\mathcal{F}_{\text {GeN[·] }}$}

$\mathcal{F}_{\text {GEN[.] }}$ interacts with the parties in $\mathcal{P}$, a dealer $\mathrm{D} \in \mathcal{P}$, and the adversary $\mathcal{S}$ and is parametrized by a commitment key ck of a double-trapdoor homomorphic commitment scheme, along with $t$.

- On receiving (sid, D, $f(\cdot), g(\cdot), h(\cdot))$ from $\mathrm{D}$ and (sid, $i, \mathrm{D}, \mathbf{C})$ from every $P_{i} \in \mathcal{P}$, the functionality verifies whether $f(\cdot), g(\cdot)$ and $h(\cdot)$ are of degree at most $t$ and $\mathbf{C} \stackrel{?}{=} \operatorname{Comm}_{\mathrm{ck}}(f(0) ; g(0), h(0))$, where $\mathbf{C}$ is received from the majority of the parties.

- If any of the above verifications fail then the functionality sends (sid, $i, \mathrm{D}$, Failure) to every $P_{i} \in \mathcal{P}$ and $\mathcal{S}$ and halts.

- Else for every $P_{i} \in \mathcal{P}$, the functionality computes the share $f_{i}=f\left(\alpha_{i}\right)$, the opening information $g_{i}=g\left(\alpha_{i}\right), h_{i}=h\left(\alpha_{i}\right)$, and the commitment $\mathbf{C}_{f_{i}, g_{i}, h_{i}}=\operatorname{Comm}_{\mathrm{ck}}\left(f_{i} ; g_{i}, h_{i}\right)$. It sends (sid, $\left.i, \mathrm{D},[s]_{i}\right)$ to every $P_{i} \in \mathcal{P}$ where $[s]_{i}=\left(f_{i}, g_{i}, h_{i},\left\{\mathbf{C}_{f_{j}, g_{j}, h_{j}}\right\}_{P_{j} \in \mathcal{P}}\right)$ and halts.

Fig. 5. The Ideal Functionality for Verifiably Generating [·]-sharing

We note that $\mathcal{F}_{\text {GEN[.] }}$ is slightly different from the standard ideal functionality (see e.g. [2]) of verifiable secret sharing (VSS) where the parties output only their shares (and not the commitment of all the shares). In most of the standard instantiations of a VSS functionality (in the computational setting), for example the Pedersen VSS [27], a public commitment of all the shares and the secret are available to the parties without violating any privacy. In order to make these commitments available to the external protocol that invokes $\mathcal{F}_{\text {Gen [.] }}$, we allow the functionality to compute and deliver the shares along with the commitments to the parties. We note, [1] introduced a similar functionality for "committed VSS" that outputs to the parties the commitment of the secret provided by the dealer due to the same motivation mentioned above.

\subsection{Supporting Sub-protocols}

Our MPC protocol also makes use of the following sub-protocols. Due to space constraints, here we only present a high level description of these protocols and state their communication complexity. The formal details of the protocols are available in Appendix C. Since we later show that our main MPC protocol that invokes these sub-protocols is UC-secure, it is not required to prove any form of security for these subprotocols separately.

(A) Protocol $\Pi_{\langle\cdot\rangle \rightarrow[\cdot]}$ (Figure 10, Appendix C) : it takes input $\langle s\rangle_{\mathcal{X}}$ for a set $\mathcal{X}$ containing at least one honest party and either produces a sharing $[s]$ (if all the parties in $\mathcal{X}$ behave honestly) or outputs one of 
the following: the identity of a single corrupted party or a pair of parties (with at least one of them being corrupted) from $\mathcal{X}$. The protocol makes use of the functionalities $\mathcal{F}_{\mathrm{GEN}[\cdot]}$ and $\mathcal{F}_{\mathrm{BC}}$.

More specifically, let $\langle s\rangle_{i}$ denote the information (namely the share, opening information and the set of commitments) of party $P_{i} \in \mathcal{X}$ corresponding to the sharing $\langle s\rangle_{\mathcal{X}}$. To achieve the goal of our protocol, there are two clear steps to perform: first, the correct commitment for each share of $s$ corresponding to its $\langle\cdot\rangle_{\mathcal{X}}$-sharing, now available to the parties in $\mathcal{X}$, is to be made available to all the parties in $\mathcal{P}$; second, each $P_{i} \in \mathcal{X}$ is required to act as a dealer and verifiably [.]-share its already committed share $s_{i}$ among $\mathcal{P}$. Note that the commitment to $s_{i}$ is included in the set of commitments that will be already available among $\mathcal{P}$ due to the first step. Clearly, once $\left[s_{i}\right]$ are generated for each $P_{i} \in \mathcal{X}$, then $[s]$ is computed as $[s]=\sum_{P_{i} \in \mathcal{X}}\left[s_{i}\right]$; this is because $s=\sum_{P_{i} \in \mathcal{X}} s_{i}$.

Now there are two steps that may lead to the failure of the protocol. First, $P_{i} \in \mathcal{X}$ may be identified

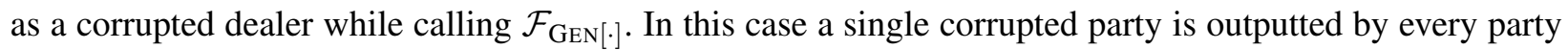
in $\mathcal{P}$. Second, the protocol may fail when the parties in $\mathcal{P}$ try to reach an agreement over the correct set of commitments of the shares of $s$. Recall that each $P_{i} \in \mathcal{X}$ holds a set of commitments as a part of $\langle s\rangle_{\mathcal{X}}$. We ask each $P_{i} \in \mathcal{X}$ to call $\mathcal{F}_{\mathrm{BC}}$ to broadcast among $\mathcal{P}$ the set of commitments held by him. It is necessary to ask each $P_{i} \in \mathcal{X}$ to do this as we can not trust any single party from $\mathcal{X}$, since all we know (with overwhelming probability) is that $\mathcal{X}$ contains at least one honest party. Now if the parties in $\mathcal{P}$ receive the same set of commitments from all the parties in $\mathcal{X}$, then clearly the received set is the correct set of commitments and agreement on the set is reached among $\mathcal{P}$. If this does not happen the parties in $\mathcal{P}$ can detect a pair of parties with conflicting sets and output the said pair. It is not hard to see that indeed one party in the pair must be corrupted. To ensure an agreement on the selected pair when there are multiple such conflicting pairs, we assume the existence of a predefined publicly known algorithm to select a pair from the lot (for instance consider the pair $\left(P_{a}, P_{b}\right)$ with minimum value of $\left.a+n \cdot b\right)$. Intuitively the protocol is secure as the shares of honest parties in $\mathcal{X}$ remain secure.

The communication complexity of protocol $\Pi_{\langle\cdot\rangle \rightarrow[\cdot]}$ is stated in Lemma 1 , which easily follows from the fact that each party in $\mathcal{X}$ needs to broadcast $\mathcal{O}(|\mathcal{X}| \kappa)$ bits to $\mathcal{P}$.

Lemma 1. The communication complexity of protocol $\Pi_{\langle\cdot\rangle \rightarrow[\cdot]}$ is $\mathcal{B C}\left(|\mathcal{X}|^{2} \kappa, n\right)$ plus the complexity of $\mathcal{O}(|\mathcal{X}|)$ invocations to the realization of the functionality $\mathcal{F}_{\mathrm{GEN}[\cdot] \cdot}$.

(B) Protocol $\Pi_{\langle\cdot\rangle}$ (Figure 11, Appendix C) : the protocol enables a designated party (dealer) D $\in \mathcal{P}$ to verifiably $\langle\cdot\rangle$-share an already committed secret $f$ among a set of parties $\mathcal{X}$ containing at least one honest party. More specifically, every $P_{i} \in \mathcal{P}$ holds a (publicly known) commitment $\mathbf{C}_{f, g, h}$. The dealer $\mathrm{D}$ holds the secret $f \in \mathbb{F}_{p}$ and randomness pair $(g, h)$, such that $\mathbf{C}_{f, g, h}=\operatorname{Comm}_{\mathrm{ck}}(f ; g, h)$; and the goal is to generate $\langle f\rangle_{\mathcal{X}}$. In the protocol, D first additively shares $f$ as well as the opening information $(g, h)$ among $\mathcal{X}$. In addition, $\mathrm{D}$ is also asked to publicly commit each additive-share of $f$, using the corresponding additive-share of $(g, f)$. The parties can then publicly verify whether indeed D has $\langle\cdot\rangle$-shared the same $f$ as committed in $\mathbf{C}_{f, g, h}$, via the homomorphic property of the commitments. Intuitively $f$ remains private in the protocol for an honest $\mathrm{D}$ as there exists at least one honest party in $\mathcal{X}$. Moreover the binding property of the commitment ensures that a potentially corrupted $\mathrm{D}$ fails to $\langle\cdot\rangle$-share an incorrect value $f^{\prime} \neq f$.

If we notice carefully the protocol achieves a little more than $\langle\cdot\rangle$-sharing of a secret among a set of parties $\mathcal{X}$. All the parties in $\mathcal{P}$ hold the commitments to the shares of $f$, while as per the definition of $\langle\cdot\rangle$ sharing the commitments to shares should be available to the parties in $\mathcal{X}$ alone. A closer look reveals that the public commitments to the shares of $f$ among the parties in $\mathcal{P}$ enable them to publicly verify whether $\mathrm{D}$ has indeed $\langle\cdot\rangle$-shared the same $f$ among $\mathcal{X}$ as committed in $\mathbf{C}_{f, g, h}$ via the homomorphic property of the commitments. The communication complexity of $\Pi_{\langle\cdot\rangle}$ is stated in Lemma 2. 
Lemma 2. The communication complexity of protocol $\Pi_{\langle\cdot\rangle}$ is $\mathcal{O}(|\mathcal{X}| \kappa)$ and $\mathcal{B C}(|\mathcal{X}| \kappa, n)$.

(C) Protocol $\Pi_{[\cdot] \rightarrow\langle\cdot\rangle}$ (Figure 12, Appendix C): the protocol takes as input $[s]$ for any secret $s$ and outputs $\langle s\rangle_{\mathcal{X}}$ for a designated set of parties $\mathcal{X} \subset \mathcal{P}$ containing at least one honest party.

Let $f_{1}, \ldots, f_{n}$ be the Shamir-shares of $s$. Then the protocol is designed using the following two-stage approach: (1): First each party $P_{k} \in \mathcal{P}$ acts as a dealer and verifiably $\langle\cdot\rangle$-share's its share $f_{k}$ via protocol $\Pi_{\langle\cdot\rangle} ;$ (2) Let $\mathcal{H}$ be the set of $|\mathcal{H}|>t+1$ parties $P_{k}$ who have correctly $\langle\cdot\rangle$-shared its Shamir-share $f_{k}$; without loss of generality, let $\mathcal{H}$ be the set of first $|\mathcal{H}|$ parties in $\mathcal{P}$. Since the original sharing polynomial (for [.]-sharing $s$ ) has degree at most $t$ with $s$ as its constant term, then there exists publicly known constants (namely the Lagrange's interpolation coefficients) $c_{1}, \ldots, c_{|\mathcal{H}|}$, such that $s=c_{1} f_{1}+\ldots+c_{|\mathcal{H}|} f_{|\mathcal{H}|}$. Since corresponding to each $P_{k} \in \mathcal{H}$ the share $f_{k}$ is $\langle\cdot\rangle$-shared, it follows easily that each party $P_{i} \in \mathcal{X}$ can compute $\langle s\rangle_{i}=c_{1}\left\langle f_{1}\right\rangle_{i}+\ldots+c_{|\mathcal{H}|}\left\langle f_{|\mathcal{H}|}\right\rangle_{i}$. The correctness of the protocol follows from the fact that the corrupted parties in $\mathcal{P}$ will fail to $\langle\cdot\rangle$-share an incorrect Shamir-share of $s$, thanks to the protocol $\Pi_{\langle\cdot\rangle}$. The privacy of $s$ follows from the fact that the Shamir shares of the honest parties in $\mathcal{P}$ remain private, which follows from the privacy of the protocol $\Pi_{\langle\cdot\rangle}$.

The communication complexity of the protocol $\Pi_{[\cdot] \rightarrow\langle\cdot\rangle}$ is stated in Lemma 3 which follows from the fact that $n$ invocations to $\Pi_{\langle\cdot\rangle}$ are done in the protocol.

Lemma 3. The communication complexity of $\Pi_{[\cdot] \rightarrow\langle\cdot\rangle}$ is $\mathcal{O}(n|\mathcal{X}| \kappa)$ and $\mathcal{B C}(n|\mathcal{X}| \kappa, n)$.

(D) Protocol $\Pi_{\text {Randzero[.] }}($ Figure 14, Appendix C): the protocol is used for generating a random [.]sharing of 0 . To design the protocol, we also require a standard Zero-knowledge (ZK) functionality $\mathcal{F}_{\text {ZK.BC }}$ to publicly prove a commitment to zero. The functionality is a "prove-and-broadcast " functionality that upon receiving a commitment and witness pair $(\mathbf{C},(u, v))$ from a designated prover $P_{j}$, verifies if $\mathbf{C}=$ $\operatorname{Comm}_{\mathrm{ck}}(0 ; u, v)$ or not. If so it sends $\mathbf{C}$ to all the parties. A protocol $\Pi_{\mathrm{ZK} \text {.BC }}$ realizing $\mathcal{F}_{\mathrm{ZK} . \mathrm{BC}}$ can be designed in the CRS model using standard techniques, see [22], with communication complexity $\mathcal{O}(\operatorname{Poly}(n) \kappa)$.

Protocol $\Pi_{\text {RandZero[.] }}$ invokes the ideal functionalities $\mathcal{F}_{\text {ZK.BC }}$ and $\mathcal{F}_{\text {Gen[.] }}$. The idea is as follows: Each party $P_{i} \in \mathcal{P}$ first broadcasts a random commitment of 0 and proves in a zero-knowledge (ZK) fashion that it indeed committed 0 . Next $P_{i}$ calls $\mathcal{F}_{\mathrm{GEN}[\cdot]}$ as a dealer $\mathrm{D}$ to generate [.]-sharing of 0 that is consistent with the commitment of 0 . The parties then locally add the sharings of the dealers who are successful as dealers

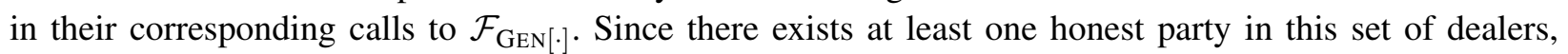
the resultant sharing will be indeed a random sharing of 0 , see Appendix $\mathrm{C}$ for details. Looking ahead, we invoke $\Pi_{\text {RANDZERo[.] }}$ only once in our main MPC protocol $\Pi_{f}$ (more on this later); so we avoid giving details of the communication complexity of the protocol. However assuming standard realization of $\mathcal{F}_{\mathrm{ZK} \text {.BC }}$, the protocol has complexity $\mathcal{O}(\operatorname{Poly}(n) \kappa)$.

(E) Dis-honest Majority MPC Protocol (Appendix D): Apart from the above sub-protocols, we use a non-robust, dishonest-majority MPC protocol $\Pi_{\mathrm{C}}^{\mathrm{NR}}$ with the capability of fault-detection. The protocol, presented in Figure 18 of Appendix D, allows a designated set of parties $\mathcal{X} \subset \mathcal{P}$, containing at least one honest party, to perform $\langle\cdot\rangle$-shared evaluation of a given circuit $C$. In case some corrupted party in $\mathcal{X}$ behaves maliciously, the parties in $\mathcal{P}$ identify a pair of parties from $\mathcal{X}$, with at least one of them being corrupted. The starting point of $\Pi_{\mathrm{C}}^{\mathrm{NR}}$ is the dishonest majority MPC protocol of [12], which takes $\langle\cdot\rangle$-shared inputs of a given circuit, from a set of parties, say $\mathcal{X}$, having a dishonest majority. The protocol then achieves the following:

- If all the parties in $\mathcal{X}$ behave honestly, then the protocol outputs $\langle\cdot\rangle$-shared circuit outputs among $\mathcal{X}$.

- Else the honest parties in $\mathcal{X}$ detect misbehaviour by the corrupted parties and abort the protocol. 
We observe that for an aborted execution of the protocol of [12], there exists an honest party in $\mathcal{X}$ that can locally identify a corrupted party from $\mathcal{X}$, who deviated from the protocol. We exploit this property in $\Pi_{\mathrm{C}}^{\mathrm{NR}}$ to enable the parties in $\mathcal{P}$ identify a pair of parties from $\mathcal{X}$ with at least one of them being corrupted.

Protocol $\Pi_{\mathrm{C}}^{\mathrm{NR}}$ proceeds in two stages, the preparation stage and the evaluation stage, each involving various other sub-protocols (details available in Appendix D). In the preparation stage, if all the parties in $\mathcal{X}$ behave honestly, then they jointly generate $C_{M}+C_{R}$ shared multiplication triples $\left\{\left(\left\langle\mathbf{a}^{(i)}\right\rangle_{\mathcal{X}},\left\langle\mathbf{b}^{(i)}\right\rangle_{\mathcal{X}}\right.\right.$,

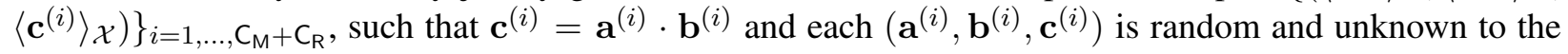
adversary; here $C_{M}$ and $C_{R}$ are the number of multiplication and random gates in $C$ respectively. Otherwise, the parties in $\mathcal{P}$ identify a pair of parties in $\mathcal{X}$, with at least one of them being corrupted.

Assuming that the desired $\langle\cdot\rangle$-shared multiplication triples are generated in the preparation stage, the parties in $\mathcal{X}$ start evaluating $\mathrm{C}$ in a shared fashion by maintaining the following standard invariant for each gate of C: Given $\langle\cdot\rangle$-shared inputs of the gate, the parties securely compute the $\langle\cdot\rangle$-shared output of the gate. Maintaining the invariant for the linear gates in $\mathrm{C}$ does not require any interaction, thanks to the linearity of $\langle\cdot\rangle$-sharing. For a multiplication gate, the parties deploy a preprocessed $\langle\cdot\rangle$-shared multiplication triple from the preparation stage (for each multiplication gate a different triple is deployed) and use the standard Beaver's trick [3], (see protocol $\Pi_{\mathrm{BEA}}$ in Appendix D). While applying Beaver's trick, the parties in $\mathcal{X}$ need to publicly open two $\langle\cdot\rangle$-shared values using a reconstruction protocol $\Pi_{\mathrm{REC}\langle\cdot\rangle}$ (presented in Appendix D). It may be possible that the opening is non-robust ${ }^{8}$, in which case the circuit evaluation fails and the parties in $\mathcal{P}$ identify a pair of parties from $\mathcal{X}$ with at least one of them being corrupted. For a random gate, the parties consider an $\langle\cdot\rangle$-shared multiplication triple from the preparation stage (for each random gate a different triple is deployed) and the first component of the triple is considered as the output of the random gate. The protocol ends once the parties in $\mathcal{X}$ obtain $\langle\cdot\rangle$-shared circuit outputs $\left\langle y_{1}\right\rangle_{\mathcal{X}}, \ldots,\left\langle y_{\text {out }}\right\rangle_{\mathcal{X}}$; so no reconstruction is required at the end.

The complete details of $\Pi_{\mathrm{C}}^{\mathrm{NR}}$ is provided in Appendix D. The protocol invokes two ideal functionalities $\mathcal{F}_{\text {GenRAND }\langle\cdot\rangle}$ and $\mathcal{F}_{\mathrm{BC}}$ where the functionality $\mathcal{F}_{\text {GenRAND }\langle\cdot\rangle}$ is used to generate $\langle\cdot\rangle$-sharing of random values (again see Appendix D). For our purpose we note that the protocol provides a statistical security of $2^{-s}$ and has communication complexity as stated in Lemma 4 and proved in Appendix D. Note that there are two types of broadcast involved: among the parties in $\mathcal{X}$ and among the parties in $\mathcal{P}$.

Lemma 4. For a statistical security parameter $s$, protocol $\Pi_{\mathrm{C}}^{\mathrm{NR}}$ has communication complexity of $\mathcal{O}\left(|\mathcal{X}|^{2}(|\mathrm{C}|+\right.$ $s) \kappa), \mathcal{B C}\left(|\mathcal{X}|^{2}(|C|+s) \kappa,|\mathcal{X}|\right)$ and $\mathcal{B C}(|\mathcal{X}| \kappa, n)$.

\subsection{The MPC Protocol}

Finally, we describe our MPC protocol. Recall that we divide the circuit ckt into sub-circuits $\mathrm{ckt}_{1}, \ldots, \mathrm{ckt}_{L}$

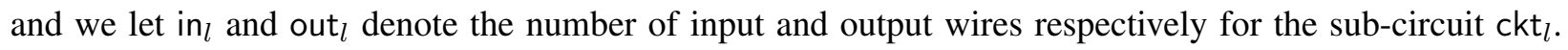
At the beginning of the protocol, each party [.]-share their private inputs by calling $\mathcal{F}_{\text {Gen }[\cdot] \text {. The parties }}$ then select a random committee of parties by calling $\mathcal{F}_{\text {Сомміттев }}$ for evaluating the $l$ th sub-circuit via the dishonest majority MPC protocol of [12]. We use a Boolean flag NewCom in the protocol to indicate if a new committee has to be decided, prior to the evaluation of $l$ th sub-circuit or the committee used for the evaluation of the $(l-1)$ th sub-circuit is to be continued. Specifically a successful evaluation of a sub-circuit is followed by setting NewCom equals to 0, implying that the current committee is to be continued for the evaluation of the subsequent sub-circuit. On the other hand, a failed evaluation of a sub-circuit is followed by setting NewCom equals to 1, implying that a fresh committee has to be decided for the re-evaluation of the same sub-circuit from the updated set of eligible parties $\mathcal{L}$, which is modified after the failed evaluation.

\footnotetext{
${ }^{8}$ As we may not have honest majority in $\mathcal{X}$, we could not always ensure robust reconstruction during $\Pi_{\operatorname{REC}\langle\cdot\rangle}$.
} 
After each successful sub-circuit evaluation, the corresponding $\langle\cdot\rangle$-shared outputs are converted into [.]shared outputs via protocol $\Pi_{\langle\cdot\rangle \rightarrow[\cdot]}$, while prior to each sub-circuit evaluation, the corresponding [.]-shared inputs are converted to the required $\langle\cdot\rangle$-shared inputs via protocol $\Pi_{[\cdot] \rightarrow\langle\cdot\rangle}$. The process is repeated till the function output is [.]-shared, after which it is robustly reconstructed (as we have honest majority in $\mathcal{P}$ ).

Without affecting the correctness of the above steps, but to ensure simulation security (in the UC model), we add an additional output re-randomization step before the output reconstruction: the parties call $\Pi_{\text {RANDZero[.] }}$ to generate a random [0], which they add to the [.]-shared output (thus keeping the same function output). Looking ahead, during the simulation in the security proof, this step allows the simulator to cheat and set the final output to be the one obtained from the functionality, even though it simulates the honest parties with 0 as the input (see Appendix E for the details).

Let $E$ be the event that at least one party in each of the selected committees during sub-circuit evaluations is honest; the event $E$ occurs except with probability at most $(t+1) \cdot \epsilon^{\mathfrak{c}} \approx 2^{-\kappa}$. This is because at most $(t+1)$ (random) committees need to be selected (a new committee is selected after each of the $t$ failed sub-circuit evaluation plus an initial selection is made). It is easy to see that conditioned on $\mathrm{E}$, the protocol is private: the inputs of the honest parties remain private during the input stage (due to $\mathcal{F}_{\text {Gen [.] }}$ ), while each of the involved sub-protocols for sub-circuit evaluations does not leak any information about honest party's inputs. It also follows that conditioned on $\mathrm{E}$, the protocol is correct, thanks to the binding property of the commitment and the properties of the involved sub-protocols.

The properties of the protocol $\Pi_{f}$ are stated in Theorem 1 and the security proof is available in Appendix E; we only provide the proof of communication complexity here. The (circuit-dependent) communication complexity in the theorem is derived after substituting the calls to the various ideal functionalities by the corresponding protocols implementing them. The broadcast complexity has two parts: the broadcasts among the parties in $\mathcal{P}$ and the broadcasts among small committees.

Theorem 1. Let $f: \mathbb{F}_{p}^{n} \rightarrow \mathbb{F}_{p}$ be a publicly known n-input function with circuit representation ckt over $\mathbb{F}_{p}$, with average width $w$ and depth $d$ (thus $w=\frac{|\mathrm{ckt}|}{d}$ ). Moreover, let $\mathrm{ckt}$ be divided into sub-circuits $\mathrm{ckt}_{1}, \ldots, \mathrm{ckt}_{L}$, with $L=t$ and each sub-circuit $\mathrm{ckt}_{l}$ having fan-in $\mathrm{in}_{l}$ and fan-out out $_{l}$. Furthermore, let $\operatorname{in}_{l}=$ out $_{l}=\mathcal{O}(w)$. Then conditioned on the event $\mathrm{E}$, protocol $\Pi_{f}(\kappa, s)$-securely realizes the functionality $\mathcal{F}_{f}$ against $\mathcal{A}$ in the $\left(\mathcal{F}_{\mathrm{CRS}}, \mathcal{F}_{\mathrm{BC}}, \mathcal{F}_{\mathrm{CommitTeE}}, \mathcal{F}_{\mathrm{GeN}[\cdot]}, \mathcal{F}_{\mathrm{GENRAND}\langle\cdot\rangle}, \mathcal{F}_{\mathrm{ZK} . \mathrm{BC}}\right)$-hybrid model in the UC security framework. The circuit-dependent communication complexity of the protocol is $\mathcal{O}\left(|\mathrm{ckt}| \cdot\left(\frac{n \cdot t}{d}+\kappa\right) \cdot \kappa^{2}\right)$, $\mathcal{B C}\left(|\mathrm{ckt}| \cdot \frac{n \cdot t \cdot \kappa^{2}}{d}, n\right)$ and $\mathcal{B C}\left(|\mathrm{ckt}| \cdot \kappa^{3}, \kappa\right)$.

PROOF (COMMUNICATION COMPLEXITY): We analyze each phase of the protocol:

1. Input Commitment Stage: Here each party broadcasts $\mathcal{O}(\kappa)$ bits to the parties in $\mathcal{P}$ and so the broadcast complexity of this step is $\mathcal{B C}(n \kappa, n)$.

2. [.]-sharing of Committed Inputs: Here $n$ calls to $\mathcal{F}_{\text {GEN [.] }}$ are made. Realizing $\mathcal{F}_{\text {GEN[.] }}$ with the protocol $\Pi_{[\cdot]}$, see Lemma 5, this incurs a communication complexity of $\mathcal{O}\left(n^{2} \kappa\right)$ and $\mathcal{B C}\left(n^{2} \kappa, n\right)$.

3. Sub-circuit Evaluations: We first count the total communication cost of evaluating the sub-circuit

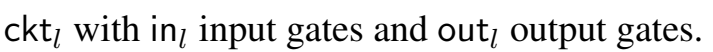

- Converting the $\operatorname{in}_{l}[\cdot]$-shared inputs to $\operatorname{in}_{l}\langle\cdot\rangle$-shared inputs will require $\operatorname{in}_{l}$ invocations to the protocol $\Pi_{[\cdot] \rightarrow\langle\cdot\rangle}$. The communication complexity of this step is $\mathcal{O}\left(n \cdot \mathfrak{c} \cdot \operatorname{in}_{l} \cdot \kappa\right)$ and $\mathcal{B C}\left(n \cdot \mathfrak{c} \cdot \operatorname{in}_{l} \cdot \kappa, n\right)$; this follows from Lemma 3 by substituting $|\mathcal{X}|=\mathfrak{c}$.

- Since the size of $\mathrm{ckt}_{l}$ is at most $\frac{|\mathrm{ckt}|}{L}$, evaluating the same via protocol $\Pi_{\mathrm{ckt}_{l}}^{\mathrm{NR}}$ will have communication complexity $\mathcal{O}\left(\mathfrak{c}^{2}\left(\frac{|\mathrm{ckt}|}{L}+s\right) \kappa\right), \mathcal{B C}\left(\mathfrak{c}^{2}\left(\frac{|\mathrm{ckt}|}{L}+s\right) \kappa, \mathfrak{c}\right)$ and $\mathcal{B C}(\mathfrak{c} \cdot \kappa, n)$; this follows from Lemma 4 by substituting $|\mathcal{X}|=\mathfrak{c}$.

\footnotetext{
${ }^{9}$ See Appendix A for the meaning of g-hybrid model in the UC framework.
} 


\section{Protocol $\Pi_{f}(\mathcal{P}, \mathrm{ckt})$}

For session ID sid, every party $P_{i} \in \mathcal{P}$ does the following:

Initialization. Set $\mathcal{L}=\mathcal{P}, \mathfrak{n}=|\mathcal{L}|, \mathfrak{t}=t$ and NewCom $=1$. Divide ckt into $L$ sub-circuits $\mathrm{ckt}_{1}, \ldots$, $\mathrm{ckt}_{L}$, each of depth $d / L$.

CRS Generation. Invoke $\mathcal{F}_{\mathrm{CRS}}$ with (sid, $i$ ) and get back (sid, $\left.i, \mathrm{CRS}\right)$, where CRS $=(\mathrm{pk}$, ck).

Input Commitment. On input $x^{(i)}$, choose random polynomials $f^{(i)}(\cdot), g^{(i)}(\cdot), h^{(i)}(\cdot)$ of degree $\leq t$, such that $f^{(i)}(0)=$

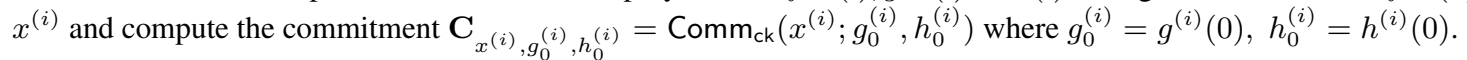

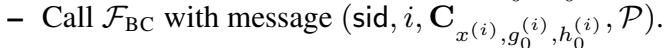

- Corresponding to each $P_{j} \in \mathcal{P}$, receive (sid, $\left.i, j, \mathbf{C}_{x^{(j)}, g_{0}^{(j)}, h_{0}^{(j)}}\right)$ from $\mathcal{F}_{\mathrm{BC}}$.

$[\cdot]$-sharing of Committed Inputs.

- Act as a dealer D and call $\mathcal{F}_{\mathrm{GeN}[\cdot]}$ with $\left(\operatorname{sid}, i, f^{(i)}(\cdot), g^{(i)}(\cdot), h^{(i)}(\cdot)\right)$.

- For every $P_{j} \in \mathcal{P}$, call $\mathcal{F}_{\mathrm{Gen}[\cdot]}$ with (sid, $\left.i, j, \mathbf{C}_{x^{(j)}, g_{0}^{(j)}, h_{0}^{(j)}}\right)$.

- For every $P_{j} \in \mathcal{P}$, if (sid, $i, j$, Failure) is received from $\mathcal{F}_{\mathrm{GeN}[\cdot]}$, substitute a default predefined public sharing [0] of 0 as $\left[x^{(j)}\right]$, set $\left[x^{(j)}\right]_{i}=[0]_{i}$ and update $\mathcal{L}=\mathcal{L} \backslash\left\{P_{j}\right\}$, decrement $\mathfrak{t}$ and $\mathfrak{n}$ by one. Else receive $\left(\operatorname{sid}, i, j,\left[x^{(j)}\right]_{i}\right)$ from $\mathcal{F}_{\mathrm{GeN}[\cdot] \cdot}$.

Start of While Loop Over the Sub-circuits. Initialize $l=1$. While $l \leq L$ do:

- Committee Selection. If NewCom $=1$, then call $\mathcal{F}_{\text {Committee }}$ with $(\operatorname{sid}, i, \mathcal{L})$ and receive $(\operatorname{sid}, i, \mathcal{C})$ from $\mathcal{F}_{\text {CommitтеE. }}$

- [.] to $\langle\cdot\rangle_{\mathcal{C}}$ Conversion of Inputs of Sub-circuit $\mathrm{ckt}_{l}$. Let $\left[x_{1}\right], \ldots,\left[x_{\mathrm{in}_{l}}\right]$ denote the $[\cdot]$-sharing of the inputs to $\mathrm{ckt}_{l}$ :

- For $k=1, \ldots$, in $_{l}$, participate in $\Pi_{[\cdot] \rightarrow\langle\cdot\rangle}$ with $\left(\operatorname{sid}, i,\left[x_{k}\right]_{i}, \mathcal{C}\right)$. Output $\left(\right.$ sid, $\left.i,\left\langle x_{k}\right\rangle_{i}\right)$ in $\Pi_{[\cdot] \rightarrow\langle\cdot\rangle}$, if $P_{i}$ belongs to $\mathcal{C}$. Else output $($ sid, $i)$.

- Evaluation of the Sub-circuit $\mathrm{ckt}_{l}$. If $P_{i} \in \mathcal{C}$ then participate in $\Pi_{\mathrm{ckt}_{l}}^{\mathrm{NR}}$ with $\left(\right.$ sid, $\left.i,\left\langle x_{1}\right\rangle_{i}, \ldots,\left\langle x_{\mathrm{in}_{l}}\right\rangle_{i}, \mathcal{C}\right)$, else participate in $\Pi_{\mathrm{ckt}_{l}}^{\mathrm{NR}}$ with $(\mathrm{sid}, i, \mathcal{C})$.

- If (sid, $i$, Failure, $\left.P_{a}, P_{b}\right)$ is the output during $\Pi_{\mathrm{ckt}_{l}}^{\mathrm{NR}}$, then set $\mathcal{L}=\mathcal{L} \backslash\left\{P_{a}, P_{b}\right\}, \mathfrak{t}=\mathfrak{t}-1, \mathfrak{n}=\mathfrak{n}-2$, NewCom $=1$ and go to Committee Selection step.

$-\langle\cdot\rangle_{\mathcal{C}}$ to $[\cdot]$ conversion of Outputs of $\mathrm{ckt}_{l}$. If (sid, $i$, Success, $\left.\left\langle y_{1}\right\rangle_{i}, \ldots,\left\langle y_{\mathrm{out}_{l}}\right\rangle_{i}\right)$ or (sid, $i$, Success) is obtained during $\Pi_{\mathrm{ckt}_{l}}^{\mathrm{NR}}$, then participate in $\Pi_{\langle\cdot\rangle \rightarrow[\cdot]}$ with $\left(\right.$ sid, $\left.i,\left\langle y_{k}\right\rangle_{i}\right)$ or (sid, $\left.i\right)$ (respectively) for $k=1, \ldots$, out . $_{l}$.

- If (sid, $i$, Success, $\left.\left[y_{k}\right]_{i}\right)$ is the output in $\Pi_{\langle\cdot\rangle \rightarrow[\cdot]}$ for every $k=1, \ldots$, out ${ }_{l}$, then increment $l$ and set NewCom $=0$.

- If (sid, $i$, Failure, $P_{a}, P_{b}$ ) is the output in $\Pi_{\langle\cdot\rangle \rightarrow[\cdot]}$ for some $k \in\left\{1, \ldots\right.$, out $\left.{ }_{l}\right\}$, then set $\mathcal{L}=\mathcal{L} \backslash\left\{P_{a}, P_{b}\right\}$, $\mathfrak{t}=\mathfrak{t}-1, \mathfrak{n}=\mathfrak{n}-2$, NewCom $=1$ and go to the Committee Selection step.

- If (sid, $i$, Failure, $\left.P_{a}\right)$ is the output in $\Pi_{\langle\cdot\rangle \rightarrow[\cdot]}$ for some $k \in\left\{1, \ldots\right.$, out $\left.{ }_{l}\right\}$, then set $\mathcal{L}=\mathcal{L} \backslash\left\{P_{a}\right\}, \mathfrak{t}=\mathfrak{t}-1$, $\mathfrak{n}=\mathfrak{n}-1$, NewCom $=1$ and go to the Committee Selection step.

Output Rerandomization. Let $[y]$ denote the [.]-sharing of the output of ckt. Participate in $\Pi_{\text {RandZero }[\cdot]}$ with $($ sid, $i)$, obtain $\left(\right.$ sid, $\left.i,[0]_{i}\right)$ and locally compute $[z]_{i}=[y]_{i}+[0]_{i}$.

Output Reconstruction. Interpret $[z]_{i}$ as $\left(f_{i}, g_{i}, h_{i},\left\{\mathbf{C}_{f_{j}, g_{j}, h_{j}}\right\}_{P_{j} \in \mathcal{P}}\right)$. Initialize a set $\mathcal{T}_{i}$ to $\emptyset$.

- Send (sid, $\left.i, j, f_{i}, g_{i}, h_{i}\right)$ to every $P_{j} \in \mathcal{P}$. On receiving (sid, $j, i, f_{j}, g_{j}, h_{j}$ ) from every party $P_{j}$ include party $P_{j}$ in $\mathcal{T}_{i}$ if $\mathbf{C}_{f_{j}, g_{j}, h_{j}} \neq \operatorname{Comm}_{\mathrm{ck}}\left(f_{j} ;\left(g_{j}, h_{j}\right)\right)$.

- Interpolate $f(\cdot)$ such that $f\left(\alpha_{j}\right)=f_{j}$ holds for every $P_{j} \in \mathcal{P} \backslash \mathcal{T}_{i}$. If $f(\cdot)$ has degree at most $t$, output (sid, $i, z=f(0))$ and halt; else output (sid, $i$, Failure) and halt.

Fig. 6. Protocol for UC-secure realizing $\mathcal{F}_{f}$ 


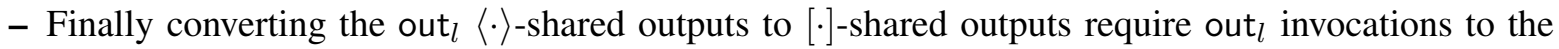
protocol $\Pi_{\langle\cdot\rangle \rightarrow[\cdot]}$. This has communication complexity $\mathcal{O}\left(n \cdot \mathfrak{c} \cdot\right.$ out $\left._{l} \cdot \kappa\right), \mathcal{B C}\left(\right.$ out $\left._{l} \cdot \mathfrak{c}^{2} \cdot \kappa, n\right)$ and $\mathcal{B C}(n \cdot \mathfrak{c} \cdot \kappa, n)$; this follows from Lemma 1 by substituting $|\mathcal{X}|=\mathfrak{c}$.

Thus evaluating $\mathrm{ckt}_{l}$ has communication complexity $\mathcal{O}\left(\left(n^{2}+n \cdot \mathfrak{c} \cdot \mathrm{in}_{l}+n \cdot \mathfrak{c} \cdot\right.\right.$ out $\left.\left._{l}+\mathfrak{c}^{2}\left(\frac{|\mathrm{ckt}|}{L}+s\right)\right) \kappa\right)$, $\mathcal{B C}\left(\left(n^{2}+n \cdot \mathfrak{c} \cdot \mathrm{in}_{l}+\mathfrak{c}^{2} \cdot\right.\right.$ out $\left.\left._{l}\right) \kappa, n\right)$ and $\mathcal{B C}\left(\mathfrak{c}^{2}\left(\frac{|\mathrm{ckt}|}{L}+s\right) \kappa, \mathfrak{c}\right)$. Assuming $\operatorname{in}_{l}=\mathcal{O}(w)$ and out ${ }_{l}=\mathcal{O}(w)$, with $w=\frac{|\mathrm{ckt}|}{d}$, this results in $\mathcal{O}\left(\left(n^{2}+n \cdot \mathfrak{c} \cdot \frac{|\mathrm{ckt}|}{d}+\mathfrak{c}^{2}\left(\frac{|\mathrm{ckt}|}{L}+s\right)\right) \kappa\right), \mathcal{B C}\left(\left(n^{2}+n \cdot \mathfrak{c} \cdot \frac{|\mathrm{ckt}|}{d}\right) \kappa, n\right)$ and $\mathcal{B C}\left(\left(\mathfrak{c}^{2} \cdot\left(\frac{|c k t|}{L}+s\right)\right) \kappa, \mathfrak{c}\right)$. The total number of sub-circuit evaluations is at most $L+t$, with $L$ successful evaluations and at most $t$ failed evaluations. Substituting $L=t$, we get the overall communication complexity $\mathcal{O}\left(\left(|\mathrm{ckt}| \cdot\left(\frac{n \cdot t \cdot \mathfrak{c}}{d}+\mathfrak{c}^{2}\right)+n^{2} t+\mathfrak{c}^{2} s \cdot t\right) \kappa\right), \mathcal{B C}\left(\left(|\mathrm{ckt}| \cdot \frac{n \cdot t \cdot \mathfrak{c}}{d}+n^{2} t\right) \kappa, n\right)$ and $\mathcal{B C}\left(\left(|\mathrm{ckt}| \cdot \mathfrak{c}^{2}+\right.\right.$ $\left.\left.\mathfrak{c}^{2} \cdot s \cdot t\right) \kappa, \mathfrak{c}\right)$.

4. Output Rerandomization and Reconstruction: The costs $\mathcal{O}(\operatorname{Poly}(n, \kappa))$ bits.

The circuit-dependent complexity of the whole protocol comes out to be $\mathcal{O}\left(|c k t| \cdot\left(\frac{n t \cdot \mathfrak{c}}{d}+\mathfrak{c}^{2}\right) \kappa\right)$ bits of communication over the point-to-point channels and broadcast-complexity of $\mathcal{B C}\left(|c k t| \cdot \frac{n t \cdot \mathfrak{c}}{d} \cdot \kappa, n\right)$ and $\mathcal{B C}\left(|c k t| \cdot \mathfrak{c}^{2} \cdot \kappa, \mathfrak{c}\right)$. Since $\mathfrak{c}$ has to be selected so that $\epsilon^{\mathfrak{c}}<2^{-\kappa}$ holds, asymptotically we can set $\mathfrak{c}$ to be $\mathcal{O}(\kappa)$. (For any practical purpose, $\kappa=80$ is good enough.) It implies that the (circuit-dependent) communication complexity is $\mathcal{O}\left(|\mathrm{ckt}|\left(\frac{n t}{d}+\kappa\right) \kappa^{2}\right), \mathcal{B C}\left(|\mathrm{ckt}| \cdot \frac{n t \kappa^{2}}{d}, n\right)$ and $\mathcal{B C}\left(|\mathrm{ckt}| \kappa^{3}, \kappa\right)$.

We propose two optimizations for our MPC protocol that improves its communication complexity.

[·]-sharing among a smaller subset of $\mathcal{P}$. While for simplicity, we involve the entire set of parties in $\mathcal{P}$ to hold [.]-shared values in the protocol, it is enough to fix and involve a set of just $z$ parties that guarantees a honest majority with overwhelming probability. From our analysis in Section 1, we find that $z=\mathcal{O}(\kappa)$. Indeed it is easy to note that all we require from the set involved in holding a [.]-sharing is honest majority that can be attained by any set containing $\mathcal{O}(\kappa)$ parties. This optimization replaces $n$ by $\kappa$ in the complexity expressions mentioned in Theorem 1. It implies that the (circuit-dependent) communication complexity is $\mathcal{O}\left(|\mathrm{ckt}|\left(\frac{\kappa t}{d}+\kappa\right) \kappa^{2}\right), \mathcal{B C}\left(|\mathrm{ckt}| \cdot \frac{t \kappa^{3}}{d}, \kappa\right)$ and $\mathcal{B C}\left(|\mathrm{ckt}| \kappa^{3}, \kappa\right)$. Now instantiating the broadcast functionality in the above modified protocol with the Dolev-Strong (DS) broadcast protocol ( see Appendix B), we obtain the following:

Corollary 1. If $d=\omega(t)$ and if the calls to $\mathcal{F}_{\mathrm{BC}}$ are realized via the DS broadcast protocol, then the circuit-dependent communication complexity of $\Pi_{f}$ is $\mathcal{O}\left(|\mathrm{ckt}| \cdot \kappa^{7}\right)$.

When we restrict to widths $w$ of the form $w=\omega\left(\kappa^{3}\right)$, we can instantiate all the invocations to $\mathcal{F}_{\mathrm{BC}}$ in the protocols $\Pi_{\langle\cdot\rangle \rightarrow[\cdot]}$ and $\Pi_{[\cdot] \rightarrow\langle\cdot\rangle}$ (invoked before and after the sub-circuit evaluations) by the Fitzi-Hirt (FH) multi-valued broadcast protocol [19], see Appendix B. This is because, setting $w=\omega\left(\kappa^{3}\right)$ ensures that the combined message over all the instances of $\Pi_{\langle\cdot\rangle \rightarrow[\cdot]}$ (respectively $\Pi_{[\cdot] \rightarrow\langle\cdot\rangle}$ ) to be broadcast by any party satisfies the bound on the message size of the FH protocol. Incorporating the above, we obtain the following corollary with better result.

Corollary 2. If $d=\omega(t)$ and $w=\omega\left(\kappa^{3}\right)$ (i.e. $|\mathrm{ckt}|=\omega\left(\kappa^{3} t\right)$ ), then the circuit-dependent communication complexity of $\Pi_{f}$ is $\mathcal{O}\left(|\mathrm{ckt}| \cdot \kappa^{4}\right)$.

Packed Secret-Sharing. We can employ packed secret-sharing technique of [20] to checkpoint multiple outputs of the sub-circuits together in a single [.]-sharing. Specifically, if we involve all the parties in $\mathcal{P}$ to hold a [·]-sharing, we can pack $n-2 t$ values together in a single $[\cdot]$-sharing by setting the degree of the underlying polynomials to $n-t-1$. It is easy to note that robust reconstruction of such a [.]-sharing is still possible, as there are $n-t$ honest parties in the set $\mathcal{P}$ and exactly $n-t$ shares are required to reconstruct an 
$(n-t-1)$ degree polynomial. For every sub-circuit $\mathrm{ckt}_{l}$, the $w_{\mathrm{out}_{l}}$ output values are grouped so that each group contains $n-2 t$ secrets and each group is then converted to a single [·]-sharing.

If we restrict to circuits for which any circuit wire has length at most $d / L=d / t$ (i.e. reaches upto at most $d / L$ levels), then we ensure that the outputs of circuit $\mathrm{ckt}_{l}$ can only be the input to circuit ckt $\mathrm{c}_{l+1}$. With this restriction, the use of packed secret-sharing becomes applicable at all stages, and the communication complexity becomes $\mathcal{O}\left(|\mathrm{ckt}| \cdot\left(\frac{t}{d}+\kappa\right) \cdot \kappa^{2}\right), \mathcal{B C}\left(|\mathrm{ckt}| \cdot \frac{t \cdot \kappa^{2}}{d}, n\right)$ and $\mathcal{B C}\left(|\mathrm{ckt}| \cdot \kappa^{3}, \kappa\right)$; i.e. a factor of $n$ less in the first two terms compared to what is stated in Theorem 1. Realizing the broadcasts using DS and FH protocol respectively, we obtain the following corollaries:

Corollary 3. If $d=\omega\left(\frac{n^{3} \cdot t}{\kappa^{4}}\right)$ and if the calls to $\mathcal{F}_{\mathrm{BC}}$ are realized via the DS broadcast protocol, then the circuit-dependent communication complexity of $\Pi_{f}$ is $\mathcal{O}\left(|\mathrm{ckt}| \cdot \kappa^{7}\right)$.

Corollary 4. If $d=\omega\left(\frac{n \cdot t}{\kappa^{5}}\right)$ and $w=\omega\left(n^{2} \cdot(n+\kappa)\right)$ (i.e. $\left.|\mathrm{ckt}|=\omega\left(\frac{n^{3} \cdot t}{\kappa^{5}}(n+\kappa)\right)\right)$, then the circuit-dependent communication complexity of the protocol $\Pi_{f}$ is $\mathcal{O}\left(|\mathrm{ckt}| \cdot \kappa^{7}\right)$.

\section{Acknowledgements}

This work has been supported in part by ERC Advanced Grant ERC-2010-AdG-267188-CRIPTO, by EPSRC via grant EP/I03126X, and by Defense Advanced Research Projects Agency (DARPA) and the Air Force Research Laboratory (AFRL) under agreement number FA8750-11-2-007910 and the third author was supported in part by a Royal Society Wolfson Merit Award.

\section{References}

1. M. Abe and S. Fehr. Adaptively Secure Feldman VSS and Applications to Universally-Composable Threshold Cryptography. In Advances in Cryptology - CRYPTO 2004, volume 3152 of LNCS, pages 317-334, 2004.

2. G. Asharov and Y. Lindell. A Full Proof of the BGW Protocol for Perfectly-Secure Multiparty Computation. IACR Cryptology ePrint Archive, 2011:136, 2011.

3. D. Beaver. Efficient Multiparty Protocols Using Circuit Randomization. In Advances in Cryptology - CRYPTO '91, volume 576 of $L N C S$, pages 420-432, 1991.

4. Z. BeerliováTrubíniová and M. Hirt. Perfectly-Secure MPC with Linear Communication Complexity. In Theory of Cryptography - TCC 2008, volume 4948 of LNCS, pages 213-230, 2008.

5. E. Ben-Sasson, S. Fehr, and R. Ostrovsky. Near-Linear Unconditionally-Secure Multiparty Computation with a Dishonest Minority. In Advances in Cryptology - CRYPTO 2012, volume 7417 of LNCS, pages 663-680, 2012.

6. E. Boyle, S. Goldwasser, and S. Tessaro. Communication locality in secure multi-party computation how to run sublinear algorithms in a distributed setting. In Theory of Cryptography - TCC 2014, volume 8349 of LNCS, pages 356-376, 2014.

7. R. Canetti. Universally Composable Security: A New Paradigm for Cryptographic Protocols. In FOCS, pages 136-145, 2001.

8. R. Canetti and M. Fischlin. Universally Composable Commitments. In Advances in Cryptology - CRYPTO 2001, pages 19-40, 2001.

9. J. F. Canny and S. Sorkin. Practical large-scale distributed key generation. In Advances in Cryptology - EUROCRYPT 2004 , volume 3027 of $L N C S$, pages 138-152, 2004.

10. A. Choudhury. Breaking the $\mathcal{O}(n|c|)$ barrier for unconditionally secure asynchronous multiparty computation - (extended abstract). In Progress in Cryptology - INDOCRYPT 2013, volume 8250 of LNCS, pages 19-37, 2013.

11. I. Damgård, Y. Ishai, M. Krøigaard, J.B. Nielsen, and A. Smith. Scalable Multiparty Computation with Nearly Optimal Work and Resilience. In Advances in Cryptology - CRYPTO 2008, volume 5157 of LNCS, pages 241-261, 2008.

12. I. Damgård and C. Orlandi. Multiparty Computation for Dishonest Majority: From Passive to Active Security at Low Cost. In Advances in Cryptology - CRYPTO 2010, volume 6223 of LNCS, pages 558-576, 2010.

\footnotetext{
${ }^{10}$ The US Government is authorized to reproduce and distribute reprints for Government purposes notwithstanding any copyright notation thereon. The views and conclusions contained herein are those of the authors and should not be interpreted as necessarily representing the official policies or endorsements, either expressed or implied, of Defense Advanced Research Projects Agency (DARPA) or the U.S. Government.
} 
13. I. Damgård, V. Pastro, N.P. Smart, and S. Zakarias. Multiparty Computation from Somewhat Homomorphic Encryption. In Advances in Cryptology - CRYPTO 2012, volume 7417 of LNCS, pages 643-662, 2012.

14. V. Dani, V. King, M. Movahedi, and J. Saia. Brief Announcement: Breaking the $O(n m)$ Bit Barrier, Secure Multiparty Computation with a Static Adversary. In Principles of Distributed Computing - PODC 2012, pages 227-228, 2012.

15. V. Dani, V. King, M. Movahedi, and J. Saia. Quorums quicken queries: Efficient asynchronous secure multiparty computation. In ICDN 2014, volume 8314 of $L N C S$, pages 242-256, 2014.

16. D. Dolev and H. R. Strong. Authenticated Algorithms for Byzantine Agreement. SIAM J. Comput., 12(4):656-666, 1983.

17. Uriel Feige. Noncryptographic selection protocols. In FOCS, pages 142-153, 1999.

18. M. Fitzi. Generalized Communication and Security Models in Byzantine Agreement. PhD thesis, ETH Zurich, 2002. ftp: //ftp.inf.ethz.ch/pub/crypto/publications/Fitzi03.pdf.

19. Matthias Fitzi and Martin Hirt. Optimally Efficient Multi-valued Byzantine Agreement. In Principles of Distributed Computing - PODC 2006, pages 163-168. ACM, 2006.

20. M. K. Franklin and M. Yung. Communication Complexity of Secure Computation (Extended Abstract). In Symposium on Theory of Computing - STOC 1992, pages 699-710. ACM, 1992.

21. R. Gennaro, M. O. Rabin, and T. Rabin. Simplified VSS and Fact-Track Multiparty Computations with Applications to Threshold Cryptography. In Principles of Distributed Computing - PODC '98, pages 101-111. ACM, 1998.

22. O. Goldreich. The Foundations of Cryptography - Volume 2, Basic Applications. Cambridge University Press, 2004.

23. B. M. Kapron, D. Kempe, V. King, J. Saia, and V. Sanwalani. Fast Asynchronous Byzantine Agreement and Leader Election with Full Information. ACM Transactions on Algorithms, 6(4), 2010.

24. V. King, S. Lonargan, J. Saia, and A. Trehan. Load Balanced Scalable Byzantine Agreement through Quorum Building, with Information. In ICDCN, volume 6522 of $L N C S$, pages 203-214, 2011.

25. V. King and J. Saia. Breaking the $O\left(n^{2}\right)$ Bit Barrier: Scalable Byzantine Agreement with an Adaptive Adversary. J. ACM, 58(4):18, 2011.

26. V. King, J. Saia, V. Sanwalani, and E. Vee. Scalable Leader Election. In SODA, pages 990-999, 2006.

27. T. P. Pedersen. Non-Interactive and Information-Theoretic Secure Verifiable Secret Sharing. In Advances in Cryptology CRYPTO '91, volume 576 of $L N C S$, pages 129-140, 1992.

28. A. Shamir. How to Share a Secret. Commun. ACM, 22(11):612-613, 1979.

29. D. R. Stinson. Cryptography - Theory and Practice. Discrete mathematics and its applications series. CRC Press, 2005.

\section{Appendices}

\section{A The UC Security Model}

We work in the standard Universal Composability (UC) framework of Canetti [7], with static corruption. The UC framework introduces a PPT environment $\mathcal{Z}$ that is invoked on the computational security parameter $\kappa$, the statistical security parameter $s$ and an auxiliary input $z$ and oversees the execution of a protocol in one of the two worlds. The "ideal" world execution involves dummy parties $P_{1}, \ldots, P_{n}$, an ideal adversary $\mathcal{S}$ who may corrupt some of the dummy parties, and a functionality $\mathcal{F}$. The "real" world execution involves the PPT parties $P_{1}, \ldots, P_{n}$ and a real world adversary $\mathcal{A}$ who may corrupt some of the parties. In either of these two worlds, a PPT adversary can corrupt $t$ parties out of the $n$ parties. The environment $\mathcal{Z}$ chooses the input of the parties and may interact with the ideal world/real world adversary during the execution. At the end of the execution, it has to decide upon and output whether a real or an ideal world execution has taken place.

We let $\operatorname{IDEAL}_{\mathcal{F}, \mathcal{S}, \mathcal{Z}}(\kappa, s, z)$ denote the random variable describing the output of the environment $\mathcal{Z}$ after interacting with the ideal execution with adversary $\mathcal{S}$, the functionality $\mathcal{F}$, on the computational security parameter $\kappa$, the statistical security parameter $s$ and $z$. Let $\operatorname{IDEAL}_{\mathcal{F}, \mathcal{S}, \mathcal{Z}}$ denote the ensemble $\left\{\operatorname{IDEAL}_{\mathcal{F}, \mathcal{S}, \mathcal{Z}}(\kappa, s, z)\right\}_{\kappa, s \in \mathbb{N}, z \in\{0,1\}^{*}}$. Similarly let $\operatorname{REAL}_{\Pi, \mathcal{A}, \mathcal{Z}}(\kappa, s, z)$ denote the random variable describing the output of the environment $\mathcal{Z}$ after interacting in a real execution of a protocol $\Pi$ with adversary $\mathcal{A}$, the parties $\mathcal{P}$, on the computational security parameter $\kappa$, the statistical security parameter $s$ and $z$. Let $\operatorname{REAL}_{\Pi, \mathcal{A}, \mathcal{Z}}$ denote the ensemble $\left\{\operatorname{REAL}_{\Pi, \mathcal{A}, \mathcal{Z}}(\kappa, s, z)\right\}_{\kappa, s \in \mathbb{N}, z \in\{0,1\}^{*}}$. 
Definition 4. For $n \in \mathbb{N}$, let $\mathcal{F}$ be an $n$-ary functionality and let $\Pi$ be an $n$-party protocol. We say that $\Pi(\kappa, s)$-securely realizes $\mathcal{F}$ in the UC security framework, if for every PPT real world adversary $\mathcal{A}$, there exists a PPT ideal world adversary $\mathcal{S}$, corrupting the same parties, such that the following two distributions are computationally indistinguishable in $\kappa$, with all but $2^{-s}$ probability:

$$
\operatorname{IDEAL}_{\mathcal{F}, \mathcal{S}, \mathcal{Z}} \stackrel{c}{\approx} \operatorname{REAL}_{\Pi, \mathcal{A}, \mathcal{Z}}
$$

We consider the above definition where it quantifies over different adversaries: passive or active, that corrupts only certain number of parties. Note that the security offered by the statistical security parameter $s$ does not depend upon the computational power of the adversary.

Modular Composition: A great advantage of the UC model is that it allows to prove the security of the protocols in a modular fashion. Specifically, the sequential modular composition theorem [7] states that in order to analyze the security of a protocol $\pi_{f}$ for computing a function $f$ that uses a subprotocol $\pi_{g}$ for computing another function $g$, it suffices to consider the execution of $\pi_{f}$ in a model where a trusted third party is used to ideally compute $g$ (instead of the parties running the real subprotocol $\pi_{g}$ ). This facilitates a modular analysis of security: we first prove the security of $\pi_{g}$ (as per the UC definition) and then prove the security of $\pi_{f}$ assuming an ideal party (functionality) for $g$. This model in which $\pi_{f}$ is analyzed using ideal calls to $g$, instead of executing $\pi_{g}$, is called the $g$-hybrid model because it involves both a real protocol execution (for computing $f$ ) and an ideal trusted third party computing $g$.

\section{B UC-secure Instantiation of Various Functionalities}

\section{B.1 Protocol for Realizing $\mathcal{F}_{\text {GeN[·] }}$}

We design a protocol $\Pi_{[\cdot]}$, presented in Figure 7, for realizing the functionality $\mathcal{F}_{\text {GeN [.] }}$ in the UC framework. We closely follow the standard Pedersen VSS scheme [27] against a threshold static adversary. Specifically, let $\mathbf{C}$ be the existing commitment available to the parties in $\mathcal{P}$ such that $\mathbf{C}=\operatorname{Comm}_{\mathrm{ck}}(s ; g, h)$ and let $(s, g, h)$ be available to D. To $[\cdot]$-share $s$, the dealer D selects three random polynomials $f(\cdot), g(\cdot)$ and $h(\cdot)$ each of degree at most $t$ such that $f(0)=s, g(0)=g$ and $h(0)=h$. To every party $P_{i}$ in $\mathcal{P}$, D distributes the share $f_{i}=f\left(\alpha_{i}\right)$ and opening information $g_{i}=g\left(\alpha_{i}\right)$ and $h_{i}=h\left(\alpha_{i}\right)$. Additionally, D publicly commits to the shares of all the share-holders, with the corresponding opening information acting as the randomness for the commitments. Namely D broadcasts $\left\{\mathbf{C}_{f_{j}, g_{j}, h_{j}}\right\}_{P_{j} \in \mathcal{P}}$ via $\mathcal{F}_{\mathrm{BC}}$.

Every honest party $P_{i}$ then verifies three conditions: (1). if the commitments correspond to polynomials of degree at most $t$ (2). if the commitments are consistent with $\mathbf{C}$ in the sense that the constant terms of the polynomials committed via the commitments $\left\{\mathbf{C}_{f_{j}, g_{j}, h_{j}}\right\}_{P_{j} \in \mathcal{P}}$ are indeed embedded in $\mathbf{C}(\mathbf{3})$. if $f_{i}, g_{i}, h_{i}$ received over the point-to-point channel is consistent with $\mathbf{C}_{f_{i}, g_{i}, h_{i}}$ received via $\mathcal{F}_{\mathrm{BC}}$. The first two tests can be done appealing to the homomorphic property of the commitment scheme. If any of the first two tests fails, then $P_{i}$ concludes that $\mathrm{D}$ is corrupted and outputs Failure. If the last test fails (but first two tests succeed), then $P_{i}$ complains $\mathrm{D}$ (publicly) who resolves the complain by revealing $f_{i}, g_{i}, h_{i}$ via $\mathcal{F}_{\mathrm{BC}}$. Subsequently, the third test is checked with $f_{i}, g_{i}, h_{i}$ received from $\mathrm{D}$ publicly. If the test is successful, $P_{i}$ accepts the new $f_{i}, g_{i}, h_{i}$ and outputs $[s]_{i}=\left(f_{i}, g_{i}, h_{i},\left\{\mathbf{C}_{f_{j}, g_{j}, h_{j}}\right\}_{P_{j} \in \mathcal{P}}\right)$. Else $P_{i}$ outputs Failure.

Intuitively the privacy of the shared secret $s$ for an honest $\mathrm{D}$ follows from the fact that $\mathcal{A}$ may learn at most $t$ shares, which constitute $t$ distinct points on $f(\cdot)$ having degree $t$; so from adversary's point of view, we have one "degree of freedom"; i.e. for every possible choice of $s$, there exists a unique $f(\cdot)$ polynomial of degree $t$, which is consistent with the shares received by $\mathcal{A}$. Note that the publicly known commitment 
of the shares do not provide any additional information about the unknown shares to $\mathcal{A}$, thanks to the (statistical) hiding property of the commitment scheme and the fact that the corresponding randomness lie on polynomials of degree at most $t$ and $\mathcal{A}$ will be provided with at most $t$ points on them, again implying one degree of freedom.

\section{Protocol $\Pi_{[\cdot]}$}

The public input to the protocol is a publicly known commitment $\mathbf{C}$ available to the parties in $\mathcal{P}$, while the private input for $\mathrm{D}$ is a secret $s$ and randomness pair $(g, h)$, such that $\mathbf{C}=\operatorname{Comm}_{\mathrm{ck}}(s ; g, h)$ holds. For session ID sid, D and the parties in $\mathcal{P}$ do the following:

Round 1 (Share Distribution and Broadcasting Commitments) - D does the following:

- Select three random polynomials $f(\cdot), g(\cdot)$ and $h(\cdot)$ of degree at most $t$, subject to the condition that $f(0)=$ $s, g(0)=g$ and $h(0)=h$.

- Corresponding to every $P_{i} \in \mathcal{P}$, compute the share $f_{i}=f\left(\alpha_{i}\right)$ and the opening information $g_{i}=g\left(\alpha_{i}\right), h_{i}=h\left(\alpha_{i}\right)$ and the commitment $\mathbf{C}_{f_{i}, g_{i}, h_{i}}=\operatorname{Comm}_{\mathrm{ck}}\left(f_{i} ; g_{i}, h_{i}\right)$.

- Corresponding to every $P_{i} \in \mathcal{P}$, send (sid, $i,\left(f_{i}, g_{i}, h_{i}\right)$ ) to the party $P_{i}$. In addition, call $\mathcal{F}_{\mathrm{BC}}$ with (sid, D, $\left.\left\{\mathbf{C}_{f_{j}, g_{j}, h_{j}}\right\}_{P_{j} \in \mathcal{P}}, \mathcal{P}\right)$.

Round 2 (Consistency Verification and Complaints) - Every party $P_{i} \in \mathcal{P}$ does the following:

- Receive (sid, $i$, D, $\left.\left(f_{i}, g_{i}, h_{i}\right)\right)$ from D and (sid, $\left.i, \mathrm{D},\left\{\mathbf{C}_{f_{j}, g_{j}, h_{j}}\right\}_{P_{j} \in \mathcal{P}}\right)$ from $\mathcal{F}_{\mathrm{BC}}$.

- Using the homomorphic property of commitments, verify

- if there exists polynomials of degree at most $t$, say $f^{\prime}(\cdot), g^{\prime}(\cdot)$ and $h^{\prime}(\cdot)$ such that $\mathbf{C}_{f_{j}, g_{j}, h_{j}}$ is $\operatorname{Comm}_{\mathrm{ck}}\left(f^{\prime}\left(\alpha_{j}\right) ; g^{\prime}\left(\alpha_{j}\right), h^{\prime}\left(\alpha_{j}\right)\right)^{a}$ for every $P_{j} \in \mathcal{P}$.

- whether the $\mathbf{C}$ is same as $\operatorname{Comm}_{\mathrm{ck}}\left(f^{\prime}(0) ; g^{\prime}(0), h^{\prime}(0)\right)$.

If any of the above tests fail then output (sid, $i$, Failure) and halt.

- Verify whether $\mathbf{C}_{f_{i}, g_{i}, h_{i}} \stackrel{?}{=} \operatorname{Comm}_{\mathrm{ck}}\left(f_{i} ; g_{i}, h_{i}\right)$. If the verification fails then call $\mathcal{F}_{\mathrm{BC}}$ with (sid, $i$, (Unhappy, D), $\mathcal{P}$ ).

Local Computation (at the end of Round 2) - Every party $P_{i}$ in $\mathcal{P}$ does the following:

- Construct a set $\mathcal{W}_{i}$ initialized to $\emptyset$ and add $P_{j} \in \mathcal{P}$ to $\mathcal{W}_{i}$ if corresponding to party $P_{j}$ the message (sid, $i, j$, (Unhappy, D)) is received from ${ }^{b} \mathcal{F}_{\mathrm{BC}}$.

- If $\left|\mathcal{W}_{i}\right|>t$, then output (sid, $i$, Failure) and halt.

Round 3 (Resolving Complaints) - D does the following:

- Corresponding to each $P_{i} \in \mathcal{W}_{\mathrm{D}}$, call $\mathcal{F}_{\mathrm{BC}}$ with the message (sid, D, (Resolve, $\left.\left.P_{i}, f_{i}, g_{i}, h_{i}\right), \mathcal{P}\right)$.

Local Computation (at the end of Round 3) - every party $P_{i} \in \mathcal{P}$ does the following:

- If there exists a $P_{k} \in \mathcal{W}_{i}$ corresponding to which the message (sid, $i$, D, (Resolve, $\left.P_{k}, f_{k}, g_{k}, h_{k}\right)$ ) is received from $\mathcal{F}_{\mathrm{BC}}$ such that $\mathbf{C}_{f_{k}, g_{k}, h_{k}} \neq \operatorname{Comm}_{\mathrm{ck}}\left(f_{k} ; g_{k}, h_{k}\right)$, then output (sid, $i$, Failure) and halt.

- Else output $[s]_{i}$ computed as follows and halt:

- If $P_{i} \in \mathcal{P} \backslash \mathcal{W}_{i}$, then $[s]_{i}=\left(f_{i}, g_{i}, h_{i},\left\{\mathbf{C}_{f_{j}, g_{j}, h_{j}}\right\}_{P_{j} \in \mathcal{P}}\right)$ where $f_{i}, g_{i}, h_{i}$ is received from D in Round 1.

- If $P_{i} \in \mathcal{W}_{i}$, then $[s]_{i}=\left(f_{i}, g_{i}, h_{i},\left\{\mathbf{C}_{f_{j}, g_{j}, h_{j}}\right\}_{P_{j} \in \mathcal{P}}\right)$ where $f_{i}, g_{i}, h_{i}$ is received from D in Round 3.

${ }^{a}$ This is done using a standard procedure based on the properties of Vandermonde matrix; see for example [21].

${ }^{b}$ The contents of $\mathcal{W}_{i}$ will be the same for each honest party $P_{i}$ in $\mathcal{P}$.

Fig. 7. Protocol for UC-secure realizing $\mathcal{F}_{\mathrm{GEN}[\cdot]}$

The properties of the protocol $\Pi_{[\cdot]}$ are formally stated in Lemma 5.

Lemma 5. Let $\mathrm{D} \in \mathcal{P}$ be a dealer with secret $s$ and randomness pair $(g, h)$ and let $\mathbf{C}$ be a publicly known commitment available to the parties in $\mathcal{P}$. Then the protocol $\Pi_{[\cdot]}$ UC-securely realizes the functionality $\mathcal{F}_{\mathrm{GEN}[\cdot]}$ in the $\mathcal{F}_{\mathrm{BC}}$-hybrid model. The protocol has communication complexity $\mathcal{O}(n \kappa)$ bits and $\mathcal{B C}(n \kappa, n)$.

PROOF: The communication complexity follows easily from the protocol. We next prove the security, considering the following two cases.

Case I - When D is honest: We first claim that in this case, no honest party will output Failure; this easily follows from the fact that an honest $\mathrm{D}$ will distribute consistent shares and only the corrupted share- 
holders (at most $t$ ) will accuse $\mathrm{D}$ and such accusations will be resolved correctly by $\mathrm{D}$. Let $T \subset \mathcal{P}$ be the set of parties under the control of $\mathcal{A}$ during the protocol $\Pi_{[\cdot]}$; we present a simulator $\mathcal{S}_{[\cdot]}^{\mathrm{HD}}$ (interacting with the functionality $\mathcal{F}_{\mathrm{GeN}[\cdot]}$ ) for $\mathcal{A}$ in Figure 8. The high level idea behind the simulator is the following: the simulator interacts with $\mathcal{F}_{\mathrm{GEN}[\cdot]}$ and obtains the shares and opening information of the corrupted parties, along with all the committed shares and sends the same to the real-world adversary; the simulator then simulates the rest of the protocol steps of $\Pi_{[\cdot]}$ on the behalf of the honest parties (including D). Any accusation by a (corrupted) share-holder can be easily resolved by the simulator, as it knows the corresponding share and opening information (as obtained from the functionality), which it can reveal. It follows easily that the simulated view has exactly the same distribution as the view of the real-world adversary in $\Pi_{[\cdot]}$.

\section{Simulator $\mathcal{S}_{[\cdot]}^{\mathrm{HD}}$}

The simulator plays the role of the honest parties (including D) and simulates each step of the protocol $\Pi_{[\cdot]}$ as follows. The communication of the $\mathcal{Z}$ with the adversary $\mathcal{A}$ is handled as follows: Every input value received by the simulator from $\mathcal{Z}$ is written on $\mathcal{A}$ 's input tape. Likewise, every output value written by $\mathcal{A}$ on its output tape is copied to the simulator's output tape (to be read by the environment $\mathcal{Z}$ ). The simulator then does the following for the session ID sid:

- Interact with $\mathcal{F}_{\mathrm{GeN}[\cdot]}$ and obtain (sid, $\left.i,\left(f_{i}, g_{i}, h_{i},\left\{\mathbf{C}_{f_{j}, g_{j}, h_{j}}\right\}_{P_{j} \in \mathcal{P}}\right)\right)$ corresponding to every corrupted party $P_{i} \in T$.

- On the behalf of $\mathrm{D}$, send (sid, $i,\left(f_{i}, g_{i}, h_{i}\right)$ ) to $\mathcal{A}$, corresponding to every $P_{i} \in T$. In addition, send (sid, D, $i,\left\{\mathbf{C}_{f_{j}, g_{j}, h_{j}}\right\}_{P_{j} \in \mathcal{P}}$ ) to $\mathcal{A}$ on the behalf of $\mathcal{F}_{\mathrm{BC}}$, corresponding to each $P_{i} \in T$.

- On receiving (sid, $i, \mathcal{P},\left(\right.$ Unhappy, D)) as the message to $\mathcal{F}_{\mathrm{BC}}$ from $\mathcal{A}$ on the behalf of any $P_{i} \in T$, send (sid, D, $i,\left(\right.$ Resolve, $\left.\left.P_{i}, f_{i}, g_{i}, h_{i}\right)\right)$ to $\mathcal{A}$ as the message from $\mathcal{F}_{\mathrm{BC}}$ on the behalf of $\mathrm{D}$.

The simulator then outputs $\mathcal{A}$ 's output and terminate.

Fig. 8. Simulator for the adversary $\mathcal{A}$ corrupting at most $t$ parties in the set $T \subset \mathcal{P} \backslash \mathrm{D}$ in the protocol $\Pi_{[\cdot]}$.

Case 2 - When $\mathrm{D}$ is Corrupted: We first note that there exists at least $t+1$ honest parties in $\mathcal{P}$ and that there exists only a unique polynomial of degree at most $t$ passing through a set of $t+1$ or more distinct points. With these facts, we next prove the security with respect to a corrupted D. Let $T \subset \mathcal{P}$ be the set of parties under the control of $\mathcal{A}$ including $\mathrm{D}$, during the protocol $\Pi_{[\cdot]}$; we present a simulator $\mathcal{S}_{[\cdot]}^{\mathrm{CD}}$ (interacting with the functionality $\mathcal{F}_{\mathrm{GEN}[\cdot]}$ ) for $\mathcal{A}$ in Figure 9 .

It follows easily that the simulated view is computationally indistinguishable from the view of the realworld adversary; otherwise we can use the corresponding distinguisher to break the binding property of the underlying commitment scheme. 


\section{Simulator $\mathcal{S}_{[\cdot]}^{\mathrm{CD}}$}

The simulator plays the role of the honest parties and simulates each step of the protocol $\Pi_{[\cdot]}$ as follows. The communication of the $\mathcal{Z}$ with the adversary $\mathcal{A}$ is handled as follows: Every input value received by the simulator from $\mathcal{Z}$ is written on $\mathcal{A}$ 's input tape. Likewise, every output value written by $\mathcal{A}$ on its output tape is copied to the simulator's output tape (to be read by the environment $\mathcal{Z}$ ). The simulator then does the following for the session ID sid:

- Play the role of $n-|T|$ honest parties and interact with $\mathcal{A}$ as per the protocol $\Pi_{[\cdot]}$.

- If Failure is obtained during the simulated execution of the protocol due to the fact that the committed shares and the corresponding randomness do not lie on polynomials of degree at most $t$, then send three arbitrary polynomials of degree more than $t$ on the behalf of $\mathrm{D}$ to the functionality $\mathcal{F}_{\mathrm{GEN}[\cdot] \cdot}$.

- Else define three polynomials $\hat{f}(\cdot), \hat{g}(\cdot)$ and $\hat{h}(\cdot)$ of degree at most $t$, such that $\hat{f}\left(\alpha_{i}\right)=f_{i}, \hat{g}\left(\alpha_{i}\right)=g_{i}$ and $\hat{h}\left(\alpha_{i}\right)=$ $h_{i}$ holds for every honest party $P_{i} \notin T$, where $f_{i}$ and $\left(g_{i}, h_{i}\right)$ are the corresponding share and opening information respectively which are obtained by $P_{i}$ during the simulated run of ${ }^{a}$ of $\Pi_{[\cdot]}$. Then send the polynomials $\hat{f}(\cdot), \hat{g}(\cdot)$ and $\hat{h}(\cdot)$ on the behalf of $\mathrm{D}$ to $\mathcal{F}_{\mathrm{GEN}[\cdot]}$.

The simulator then outputs $\mathcal{A}$ 's output and terminate.

${ }^{a}$ Note that $\hat{f}(\cdot), \hat{g}(\cdot)$ and $\hat{h}(\cdot)$ are well defined as there exists $|n|-|T|>t+1$ honest parties in $\mathcal{P}$.

Fig. 9. Simulator for the adversary $\mathcal{A}$ corrupting at most $t$ parties in the set $T \subset \mathcal{P}$ including $\mathrm{D}$ during the protocol $\Pi_{[\cdot]}$.

\section{B.2 Protocols for Realizing $\mathcal{F}_{\text {СомmiтteE }}$ and $\mathcal{F}_{\mathrm{BC}}$}

The Committee Selection Protocol: Functionality $\mathcal{F}_{\text {Cомmiтtee }}$ can be realized using various standard ways; moreover, the functionality will be invoked at most $(t+1)$ times in our MPC protocol; $t$ times corresponding to $t$ failed sub-circuit evaluations plus once for initial selection of a committee. As this cost is independent of the circuit size $|c k t|$ (but rather Poly $(n)$ ), we give only a high level sketch of one of the possible instantiations of $\mathcal{F}_{\text {Соммітте }}$, based on a computationally secure pseudo-random number generator (PRNG) [29]. Assume we have a PRNG $\mathcal{R}_{k}(\cdot)$ with seed $k$, which outputs values in the range $1, \ldots, n$. Then each time a committee needs to be formed, the parties in $\mathcal{P}$ can agree on a random seed $k$; this can be done via standard method, say by coin-flipping (or executing an instance of $\Pi_{[\cdot]}$ on the behalf of each party). Then the parties can (locally) run $\mathcal{R}$ with the obtained key, till they obtain the desired committee. It follows via the security of $\mathcal{R}$, that the committee selected like this is indeed a uniformly random committee of parties with high probability. We can simplify further by putting up a random seed in the CRS, rather than sampling a random seed on the fly every time a committee needs to be formed.

The Broadcast Protocol: Assuming a PKI set-up, the well known Dolev-Strong (DS) broadcast protocol [16] allows a sender Sen $\in \mathcal{P}$ to reliably broadcast a message $m$ of size $\ell$ to a set of parties $\mathcal{X} \subseteq \mathcal{P}$, provided $\mathcal{X}$ has at least one honest party; the protocol can be used to realize $\mathcal{F}_{\mathrm{BC}}$. As stated in [19], using the DS protocol, it costs the parties in $\mathcal{X} \cup\{$ Sen $\}$ a total communication of $\mathcal{O}\left(|\mathcal{X}|^{3} \cdot \ell \cdot \kappa\right)$ bits over the point-topoint channels to enable the Sen to broadcast $m$ to the parties in $\mathcal{X}$. As the protocol is well known in the literature, we avoid giving the details here and instead refer the interested readers to [18] for the details. We also note that [19] suggests an improved proposal for realizing $\mathcal{F}_{\mathrm{BC}}$ with a communication complexity of $\mathcal{O}\left(|\mathcal{X}| \cdot \ell+|\mathcal{X}|^{4} \cdot(|\mathcal{X}|+\kappa)\right)$ bits, but with a restriction on the size of $\ell$, namely $\ell=\omega\left(|\mathcal{X}|^{2} \cdot(|\mathcal{X}|+\kappa)\right)$. We make use of this proposal for estimating the communication complexity of our MPC protocol in Section 3.3.

\section{Supporting Sub-Protocols}

In this appendix, we present the details for the sub-protocols which enable a number of tasks such as conversion from $[\cdot]$-sharing to $\langle\cdot\rangle$-sharing and vice-versa and generating a random $[\cdot]$-sharing of 0 . 


\section{C.1 Protocol for Converting a $\langle\cdot\rangle$-sharing to a $[\cdot]$-sharing}

Protocol $\Pi_{\langle\cdot\rangle \rightarrow[\cdot]}$ is presented in Figure 10.

Protocol $\Pi_{\langle\cdot\rangle \rightarrow[\cdot]}$

For session id sid, every party $P_{i} \in \mathcal{P}$ participates with either (sid, $\left.i,\langle s\rangle_{i}\right)$ or (sid, $\left.i\right)$ and does the following:

- If $P_{i} \in \mathcal{X}$, interpret $\langle s\rangle_{i}$ as $\left(s_{i}, u_{i}, v_{i},\left\{\mathbf{C}_{s_{j}, u_{j}, v_{j}}^{P_{i}}\right\}_{P_{j} \in \mathcal{X}}\right)$ and invoke $\mathcal{F}_{\mathrm{BC}}$ with $\left(\right.$ sid, $\left.i,\left\{\mathbf{C}_{s_{j}, u_{j}, v_{j}}^{P_{i}}\right\}_{P_{j} \in \mathcal{X}}, \mathcal{P}\right)$

- Receive (sid, $i, k,\left\{\mathbf{C}_{s_{j}, u_{j}, v_{j}}^{P_{P_{j}} \in \mathcal{X}}\right.$ ) from $\mathcal{F}_{\mathrm{BC}}$ for every $P_{k} \in \mathcal{X}$ (who acted as the sender).

- If there exists a pair of parties $P_{a}, P_{b} \in \mathcal{X}$, such that $\left\{\mathbf{C}_{s_{j}, u_{j}, v_{j}}^{P_{a}}\right\}_{P_{j} \in \mathcal{X}} \neq\left\{\mathbf{C}_{s_{j}, u_{j}, v_{j}}^{P_{P_{j}}}\right\}_{P_{j}}$, then output (sid, $i$, Failure, $\left.P_{a}, P_{b}\right)$ and halt; if there are multiple such pairs $\left(P_{a}, P_{b}\right)$ the select the one with the least index $a$ and $b$. Else set $\left\{\mathbf{C}_{s_{j}, u_{j}, v_{j}}\right\}_{P_{j} \in \mathcal{X}}=\left\{\mathbf{C}_{s_{j}, u_{j}, v_{j}}^{P_{\alpha}}\right\}_{P_{j} \in \mathcal{X}}$ to be the reference set of commitments, where $P_{\alpha}$ is the least indexed party in $\mathcal{P}$.

- If $P_{i} \in \mathcal{X}$, act as a D and call $\mathcal{F}_{\mathrm{Gen}[\cdot]}$ with $\left(\right.$ sid, $\left.i, f^{(i)}(\cdot), g^{(i)}(\cdot), h^{(i)}(\cdot)\right)$ where $f^{(i)}(\cdot), g^{(i)}(\cdot)$ and $h^{(i)}(\cdot)$ are random polynomials of degree at most $t$, subject to the condition that $f^{(i)}(0)=s_{i}, g^{(i)}(0)=u_{i}$ and $h^{(i)}(0)=v_{i}$. If $P_{i} \in \mathcal{P} \backslash \mathcal{X}$, invoke $\mathcal{F}_{\mathrm{GEN}[\cdot]}$ with (sid, $i, k, \mathbf{C}_{s_{k}, u_{k}, v_{k}}$ ) for every $P_{k} \in \mathcal{X}$, where $\mathbf{C}_{s_{k}, u_{k}, v_{k}}$ is obtained from the reference set of commitments. Receive (sid, $i, k$, Failure) or (sid, $\left.i, k,\left[s_{k}\right]_{i}\right)$ from $\mathcal{F}_{\mathrm{GeN}[\cdot]}$ for every $P_{k} \in \mathcal{X}$

- Output (sid, $i$, Failure, $P_{k}$ ) and halt if (sid, $i, P_{k}$, Failure) is received from $\mathcal{F}_{\mathrm{Gen}[\cdot]}$ corresponding to any $P_{k} \in \mathcal{X}$. Otherwise, locally compute $[s]_{i}=\sum_{P_{k} \in \mathcal{X}}\left[s_{k}\right]_{i}$, output (sid, $i$, Success, $\left.[s]_{i}\right)$ and halt.

Fig. 10. Protocol for Converting $\langle\cdot\rangle$-sharing to $[\cdot]$-sharing in the $\left(\mathcal{F}_{\mathrm{BC}}, \mathcal{F}_{\mathrm{GeN}[\cdot]}\right)$-hybrid Model

\section{C.2 Protocol for Generating $\langle\cdot\rangle$-sharing of a Committed Secret}

Protocol $\Pi_{\langle\cdot\rangle}$ is presented in Figure 11. 


\section{Protocol $\Pi_{\langle\cdot\rangle}$}

For session ID sid, every $P_{i} \in \mathcal{P}$ participates with (sid, $i, \mathrm{D}, \mathbf{C}_{f, g, h}, \mathcal{X}$ ) where $\mathbf{C}_{f, g, h}$ is a (publicly known) commitment. The dealer $\mathrm{D}$ participates with (sid, $\mathrm{D}, f, g, h, \mathcal{X})$ where $f$ is the secret and $(g, h)$ is the randomness pair, such that $\mathbf{C}=$ Comm $_{\mathrm{ck}}(f ; g, h)$. The parties in $\mathcal{P}$ do the following:

Round 1 (Share Distribution and Broadcasting Commitments): Only D does the following:

- Corresponding to every $P_{i} \in \mathcal{X}$, select a random share $s_{i}$ and a random pair of opening information $u_{i}, v_{i}$, subject to the condition that $\sum_{P_{i} \in \mathcal{X}} s_{i}=f, \sum_{P_{i} \in \mathcal{X}} u_{i}=g$ and $\sum_{P_{i} \in \mathcal{X}} v_{i}=h$, and compute the commitment $\mathbf{C}_{s_{i}, u_{i}, v_{i}}=$ Comm $_{\mathrm{ck}}\left(s_{i} ; u_{i}, v_{i}\right)$. Send (sid, $\left.i, \mathrm{D}, s_{i}, u_{i}, v_{i}\right)$ to the party $P_{i}$.

- Call $\mathcal{F}_{\mathrm{BC}}$ with (sid, D, $\left.\left\{\mathbf{C}_{s_{j}, u_{j}, v_{j}}\right\}_{P_{j} \in \mathcal{X}}, \mathcal{P}\right)$ to broadcast $\left\{\mathbf{C}_{s_{j}, u_{j}, v_{j}}\right\}_{P_{j} \in \mathcal{X}}$ to all the parties in $\mathcal{P}$.

Round 2 (Consistency Verification and Complaints): Every party $P_{i} \in \mathcal{P}$ does the following:

- Receive (sid, $\left.i, \mathrm{D},\left\{\mathbf{C}_{s_{j}, u_{j}, v_{j}}\right\}_{P_{j} \in \mathcal{X}}\right)$ from $\mathcal{F}_{\mathrm{BC}}$. Additionally if $P_{i} \in \mathcal{X}$, then receive (sid, $\left.i, \mathrm{D}, s_{i}, u_{i}, v_{i}\right)$ from $\mathrm{D}$.

- Verify if $\odot P_{j} \in \mathcal{X} \mathbf{C}_{s_{j}, u_{j}, v_{j}} \stackrel{?}{=} \mathbf{C}_{f, g, h}$ (homomorphically). If the verification fails, then output (sid, $i, \mathrm{D}$, Failure) and halt.

- If $P_{i} \in \mathcal{X}$ then verify whether $\mathbf{C}_{s_{i}, u_{i}, v_{i}} \stackrel{?}{=} \operatorname{Comm}_{\mathrm{ck}}\left(s_{i} ; u_{i}, v_{i}\right)$. If the verification fails then call $\mathcal{F}_{\mathrm{BC}}$ with (sid, $i$, (Unhappy, $i, \mathrm{D}), \mathcal{P}$ ).

- Construct a set $\mathcal{W}_{i}$ initialized to $\emptyset$ and add $P_{j} \in \mathcal{X}$ to $\mathcal{W}_{i}$ if (sid, $i, j$, (Unhappy, $\left.j, \mathrm{D}\right)$ ) is received from ${ }^{a} \mathcal{F}_{\mathrm{BC}} \operatorname{cor}_{-}$ responding to $P_{j}$.

Round 3 (Resolving Complaints): Only D does the following:

- Corresponding to each $P_{i} \in \mathcal{W}_{\mathrm{D}}$, call $\mathcal{F}_{\mathrm{BC}}$ with the message (sid, D, (Resolve, $\left.i, s_{i}, u_{i}, v_{i}\right), \mathcal{P}$ ).

Local Computation (at the end of Round 3): Every party $P_{i} \in \mathcal{P}$ does the following:

- If there exists a $P_{k} \in \mathcal{W}_{i}$ corresponding to which the message (sid, $i$, D, (Resolve, $\left.k, s_{k}, u_{k}, v_{k}\right)$ ) is received from $\mathcal{F}_{\mathrm{BC}}$ such that $\mathbf{C}_{s_{k}, u_{k}, v_{k}} \neq \operatorname{Comm}_{\mathrm{ck}}\left(s_{k} ; u_{k}, v_{k}\right)$, then output (sid, $i, \mathrm{D}$, Failure) and halt.

- Else every $P_{i} \in \mathcal{P} \backslash \mathcal{X}$ outputs (sid, $i$, D, Success) and halts, while every $P_{i} \in \mathcal{X}$ does the following:

- If $P_{i} \in \mathcal{X} \backslash \mathcal{W}_{i}$, then set $\langle f\rangle_{i}=\left(s_{i}, u_{i}, v_{i},\left\{\mathbf{C}_{s_{j}, u_{j}, v_{j}}\right\}_{P_{j} \in \mathcal{X}}\right)$, where $\left(s_{i}, u_{i}, v_{i}\right)$ was received from $\mathrm{D}$ and $\left\{\mathbf{C}_{s_{j}, u_{j}, v_{j}}\right\}_{P_{j} \in \mathcal{X}}$ was received from $\mathcal{F}_{\mathrm{BC}}$ at the end of Round 1. Output (sid, $i$, D, Success, $\langle f\rangle_{i}$ ) and halt.

- Else if $P_{i} \in \mathcal{W}_{i}$, then set $\langle f\rangle_{i}=\left(s_{i}, u_{i}, v_{i},\left\{\mathbf{C}_{s_{j}, u_{j}, v_{j}}\right\}_{P_{j} \in \mathcal{X}}\right)$, where $\left(s_{i}, u_{i}, v_{i}\right)$ was received from $\mathcal{F}_{\mathrm{BC}}$ (corresponding to D) at the end of Round 3 and $\left\{\mathbf{C}_{s_{j}, u_{j}, v_{j}}\right\}_{P_{j} \in \mathcal{X}}$ was received from $\mathcal{F}_{\mathrm{BC}}$ at the end of Round 1. Output (sid, $i$, Success, $\left.\mathrm{D},\langle f\rangle_{i}\right)$ and halt.

${ }^{a}$ The contents of $\mathcal{W}_{i}$ will be the same for each honest party $P_{i}$ in $\mathcal{P}$.

Fig. 11. Protocol $\Pi_{\langle\cdot\rangle}$ for Verifiably $\langle\cdot\rangle$-sharing an Existing Committed Secret 


\section{C.3 Protocol for Transforming [·]-sharing to $\langle\cdot\rangle$-sharing}

Protocol $\Pi_{[\cdot] \rightarrow\langle\cdot\rangle}$ is presented in Figure 12.

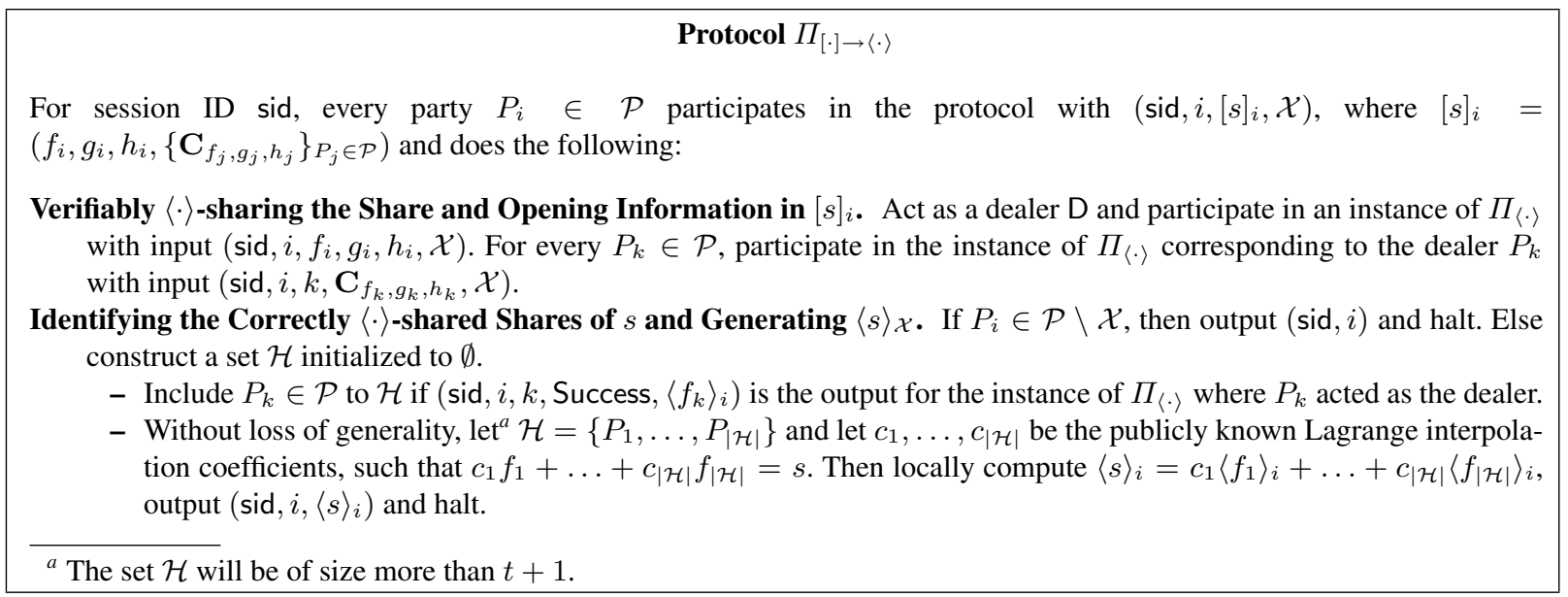

Fig. 12. Protocol $\Pi_{[\cdot] \rightarrow\langle\cdot\rangle}$ for Converting an $[\cdot]$-sharing to $\langle\cdot\rangle$-sharing.

\section{C.4 Protocol for Generating Random [-]-sharing of 0}

As mentioned earlier, the protocol uses the ideal ZK functionality $\mathcal{F}_{\mathrm{ZK} . \mathrm{BC}}$ presented in Figure 13.

Functionality $\mathcal{F}_{\text {ZK.BC }}$

$\mathcal{F}_{\text {ZK.BC }}$ interacts with a prover $P_{j} \in \mathcal{P}$ and the set of $n$ verifiers $\mathcal{P}=\left\{P_{1}, \ldots, P_{n}\right\}$ and the adversary $\mathcal{S}$ and is parameterized by the commitment key ck of a double-trapdoor homomorphic commitment scheme.

- Upon receiving (sid, $j, \mathbf{C}, u, v)$ from the prover $P_{j}$ and (sid, $\left.j, i\right)$ from every party $P_{i} \in \mathcal{P} \backslash\left\{P_{j}\right\}$, the functionality sends $($ sid, $i, \mathbf{C})$ to every party $P_{i} \in \mathcal{P}$ and $\mathcal{S}$ and halts if $\mathbf{C} \stackrel{?}{=} \operatorname{Comm}_{\mathrm{ck}}(0 ; u, v)$ is true. Else the functionality sends $($ sid, $i, \perp)$ to every party $P_{i} \in \mathcal{P}$ and $\mathcal{S}$ and halts.

Fig. 13. The Ideal Functionality for ZK Proof of Committing Zero

Now based on the functionalities $\mathcal{F}_{\text {Gen[.] }}$ and $\mathcal{F}_{\text {ZK.BC }}$, protocol $\Pi_{\text {RANDZERo[.] }}$ is presented in Figure 14. 


\section{Protocol $\Pi_{\text {RandZero[·] }}$}

For the session id sid, every party $P_{i} \in \mathcal{P}$ participates with (sid, $i$ ) and does the following:

Publicly Committing 0:

- Set $r_{i}=0$ and randomly select $u_{i}, v_{i} \in \mathbb{F}_{p}$ and compute $\mathbf{C}_{r_{i}, u_{i}, v_{i}}=\operatorname{Comm}_{\mathrm{ck}}\left(r_{i} ; u_{i}, v_{i}\right)$.

- Act as a prover and call $\mathcal{F}_{\mathrm{ZK} . \mathrm{BC}}$ with $\left(\right.$ sid, $\left.i, \mathbf{C}_{r_{i}, u_{i}, v_{i}}, u_{i}, v_{i}\right)$. Corresponding to every prover $P_{j} \in \mathcal{P} \backslash P_{i}$, participate in $\mathcal{F}_{\text {ZK. BC }}$ with $($ sid, $j, i)$.

- Construct a set $\mathcal{T}_{i}$, initialized to $\emptyset$ and include $P_{j}$ in $\mathcal{T}_{i}$ if corresponding to the prover $P_{j}$, (sid, $\left.i, \mathbf{C}_{r_{i}, u_{j}, v_{j}}\right)$ is received from $\mathcal{F}_{\text {ZK.BC. }}$.

$[\cdot]$-sharing 0 :

- Select three random polynomials $f^{(i)}(\cdot), g^{(i)}(\cdot)$ and $h^{(i)}(\cdot)$ each of degree at most $t$, subject to the condition that $f^{(i)}(0)=r_{i}, g^{(i)}(0)=u_{i}$ and $h^{(i)}(0)=v_{i}$.

- Act as a D and call $\mathcal{F}_{\mathrm{GeN}[\cdot]}$ with $\left(\operatorname{sid}, P_{i}, f^{(i)}(\cdot), g^{(i)}(\cdot), h^{(i)}(\cdot)\right)$. Corresponding to every dealer $P_{j} \in \mathcal{T}_{i}$, participate in $\mathcal{F}_{\mathrm{GeN}[\cdot]}$ with (sid, $\left.i, j, \mathbf{C}_{r_{j}, u_{j}, v_{j}}\right)$.

- If corresponding to any $P_{j} \in \mathcal{T}_{i}$, (sid, $i, P_{j}$, Failure) is received from $\mathcal{F}_{\text {Gen [.] }}$, then remove $P_{j}$ from $\mathcal{T}_{i}$.

- Locally compute $[0]_{i}=\sum_{P_{j} \in \mathcal{T}_{i}}\left[r_{j}\right]_{i}$, where (sid, $\left.i, j,\left[r_{j}\right]_{i}\right)$ is received from $\mathcal{F}_{\mathrm{GeN}[\cdot]}$ corresponding to $P_{j} \in \mathcal{T}_{i}$. Output $\left(\mathrm{sid}, i,[0]_{i}\right)$ and halt.

Fig. 14. Protocol $\Pi_{\text {RandZero[.] }}$ for generating a random [·]-sharing of 0 .

\section{Protocol $\Pi_{\mathrm{C}}^{\mathrm{NR}}$ for $\langle\cdot\rangle$-shared Evaluation of a Circuit}

As evident from the high level description of protocol $\Pi_{\mathrm{C}}^{\mathrm{NR}}$ in Section 3.2, the major step of the protocol $\Pi_{\mathrm{C}}^{\mathrm{NR}}$ is the preparation stage for generating the shared-triplets. Towards constructing the preparation stage protocol and protocol $\Pi_{\mathrm{C}}^{\mathrm{NR}}$, we begin with the building blocks and sub-protocols most of which are taken from [12] and rest are modified according to our need. Many of the sub-protocols are described with respect to a set of parties $\mathcal{X} \subset \mathcal{P}$, where we assume that $\mathcal{X}$ contains at least one honest party.

Strong Semi-honest Secure Two-party Multiplication Protocol. Protocol $\Pi_{\mathrm{MULT}}(a, b) \rightarrow\left(c_{1}, c_{2}\right)$ is a two-party protocol. The inputs of the first and second party are $a$ and $b$ respectively. The outputs to the first and second party are $c_{1}$ and $c_{2}$ respectively. It holds that $c_{1}$ is random in $\mathbb{F}_{p}$ and $c_{1}+c_{2}=a \cdot b$. Informally the protocol satisfies the following properties (for the complete formal details see [12]):

- The protocol is secure even if the adversary maliciously chooses the randomness for the corrupted parties (this is the reason [12] calls the protocol as strong semi-honest secure).

- The view of the protocol commits the adversary to his randomness and given the view and the randomness it is possible to verify whether any party deviated from the protocol.

In our context, the second property of $\Pi_{\mathrm{MULT}}$ is very crucial, as it enables an honest party involved in $\Pi_{\mathrm{MULT}}$ to identify any malicious behavior of its partner in the protocol when the individual randomness are revealed. There are various standard ways for instantiating $\Pi_{\mathrm{MULT}}(a, b)$, based on variety of standard assumptions, such as homomorphic encryption, oblivious transfer (OT), etc. An instantiation based on Paillier encryption with communication complexity $\mathcal{O}(\kappa)$ is provided in [12] (for details see [12]).

Semi-honest Secure Triple Generation Protocol. The protocol $\Pi_{\text {TRIPLE }}$ (see Figure 15) uses the two party protocol $\Pi_{\text {MULT }}$ as a sub-protocol and allows a set of parties $\mathcal{X} \subset \mathcal{P}$ to generate one $\langle\cdot\rangle$-shared multiplication triplet $\left(\langle a\rangle_{\mathcal{X}},\langle b\rangle_{\mathcal{X}},\langle c\rangle_{\mathcal{X}}\right)$. The protocol is executed assuming semi-honest adversary. The protocol is based on the following idea: every party $P_{i} \in \mathcal{X}$ selects a random $a_{i}$ and $b_{i}$ and commits the same. Then we set $a$ and $b$ to be the sum of all $a_{i}$ s and $b_{i}$ s. For setting $c$ as $a \cdot b$, every pair of parties $P_{i}, P_{j} \in \mathcal{X}$ need to securely compute the "cross-terms" $a_{i} \cdot b_{j}$ and $a_{j} \cdot b_{i}$, for which they execute two instances 
of $\Pi_{\text {MULT }}$. Once $P_{i}$ computes its $c_{i}$, it publicly commits the same. Instantiating the calls to $\Pi_{\text {MULT }}$ with that of [12] (based on the Paillier encryption), protocol $\Pi_{\text {TRIPLE }}$ has communication complexity of $\mathcal{O}\left(|\mathcal{X}|^{2} \kappa\right)$ and $\mathcal{B C}(|\mathcal{X}| \kappa,|\mathcal{X}|)$.

\section{Protocol $\Pi_{\text {TRIPLE }}$}

The public input to the protocol is a set of parties $\mathcal{X} \subset \mathcal{P}$ containing at least one honest party. For the session id sid, every party $P_{i} \in \mathcal{X}$ participates with (sid, $i$ ) and does the following:

- Randomly select shares $a_{i}, b_{i}$.

- For all $P_{j} \in \mathcal{X} \backslash P_{i}$, run $\Pi_{\mathrm{MULT}}\left(a_{i}, b_{j}\right) \rightarrow\left(d_{i j}, e_{j i}\right)$ as party 1 .

- For all $P_{j} \in \mathcal{X} \backslash P_{i}$, run $\Pi_{\mathrm{MULT}}\left(a_{j}, b_{i}\right) \rightarrow\left(d_{j i}, e_{i j}\right)$ as party 2 .

- Set $c_{i}=a_{i} \cdot b_{i}+\sum_{P_{j} \in \mathcal{X} \backslash P_{i}} d_{i j}+\sum_{P_{j} \in \mathcal{X} \backslash P_{i}} e_{i j}$.

- Randomly select $q_{i}, r_{i}, s_{i}, t_{i}, u_{i}$ and $v_{i}$ and compute the commitments $\mathbf{C}_{a_{i}, q_{i}, r_{i}}=\operatorname{Comm}_{\mathrm{ck}}\left(a_{i} ; q_{i}, r_{i}\right), \mathbf{C}_{b_{i}, s_{i}, t_{i}}=$ $\operatorname{Comm}_{\mathrm{ck}}\left(b_{i} ; s_{i}, t_{i}\right)$ and $\mathbf{C}_{c_{i}, u_{i}, v_{i}}=\operatorname{Comm}_{\mathrm{ck}}\left(c_{i} ; u_{i}, v_{i}\right)$. Call $\mathcal{F}_{\mathrm{BC}}$ with $\left(\right.$ sid, $\left.i, \mathbf{C}_{a_{i}, q_{i}, r_{i}}, \mathbf{C}_{b_{i}, s_{i}, t_{i}}, \mathbf{C}_{c_{i}, u_{i}, v_{i}}, \mathcal{X}\right)$.

- Corresponding to each $P_{j} \in \mathcal{X}$, receive (sid, $i, j, \mathbf{C}_{a_{j}, q_{j}, r_{j}}, \mathbf{C}_{b_{j}, s_{j}, t_{j}}, \mathbf{C}_{c_{j}, u_{j}, v_{j}}$ ) from $\mathcal{F}_{\mathrm{BC}}$.

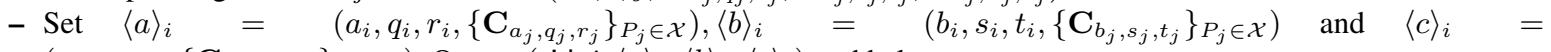

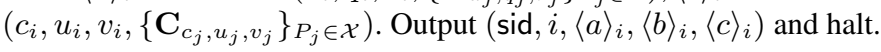

Fig. 15. Protocol for Generating One $\langle\cdot\rangle$-shared Multiplication Triple Assuming No Active Corruptions.

Functionality for Generating $\langle\cdot\rangle$-sharing of a Random Value. Functionality $\mathcal{F}_{\mathrm{GENRAND}\langle\cdot\rangle}$ (presented in Figure 16) generates an $\langle\cdot\rangle$-shared random value within a designated set of parties $\mathcal{X} \subset \mathcal{P}$, where each party in $\mathcal{X}$ "contributes" its "part" of the share and opening information for the shared random value.

Functionality $\mathcal{F}_{\text {GenRAND }\langle\cdot\rangle}$

The functionality interacts with a designated set of parties $\mathcal{X} \subset \mathcal{P}$ containing at least one honest party and the adversary $\mathcal{S}$ and is parametrized by the commitment key $\mathrm{ck}$ of a double-trapdoor commitment scheme. For the session id sid, the functionality does the following:

- On receiving (sid, $\left.i, s_{i}, u_{i}, v_{i}\right)$ from each party $P_{i} \in \mathcal{X}$, compute $s=\sum_{P_{i} \in \mathcal{X}} s_{i}, u=\sum_{P_{i} \in \mathcal{X}} u_{i}$ and $v=\sum_{P_{i} \in \mathcal{X}} v_{i}$ and $\mathbf{C}_{s, u, v}=\operatorname{Comm}_{\mathrm{ck}}(s ; u, v)$. In addition, for each $P_{i} \in \mathcal{X}$, compute $\mathbf{C}_{s_{i}, u_{i}, v_{i}}=\operatorname{Comm}_{\mathrm{ck}}\left(s_{i} ; u_{i}, v_{i}\right)$. Finally send (sid, $\left.i, \mathbf{C}_{s, u, v},\left\{\mathbf{C}_{s_{j}, u_{j}, v_{j}}\right\}_{P_{j} \in \mathcal{X}}\right)$ to every $P_{i} \in \mathcal{X}$ and halt.

Fig. 16. Functionality for Generating $\langle\cdot\rangle$-shared Random Value for a Designated Set $\mathcal{X} \subset \mathcal{P}$ with Dishonest-majority.

In [12], a realization of $\mathcal{F}_{\text {GenRAND }\langle\cdot\rangle}$ based on UC-secure multi-party commitment scheme was presented in the common reference string (CRS) model. The UC secure multi-party commitment scheme is further constructed using a CCA-secure encryption and the double-trapdoor homomorphic commitment scheme introduced in Section 2. Specifically, the following was shown; we refer to [12] for the details of the instantiation of $\mathcal{F}_{\text {GenRAnd }\langle\cdot\rangle}$.

Lemma 6 ([12]). Assuming CCA-secure encryption and double-trapdoor homomorphic commitment scheme, it is possible to $(\kappa, s)$-securely realize $\mathcal{F}_{\mathrm{GENRAND}\langle\cdot\rangle}$ in the $\left(\mathcal{F}_{\mathrm{CRS}}, \mathcal{F}_{\mathrm{BC}}\right)$-hybrid model in the UC framework. The protocol generates $\ell\langle\cdot\rangle$-shared random values and has communication complexity $\mathcal{B C}(|\mathcal{X}|(\ell+$ $s) \kappa,|\mathcal{X}|)$.

Protocol for Reconstructing $\langle\cdot\rangle$-shared Value. Protocol $\Pi_{\operatorname{Rec}\langle\cdot\rangle}$ takes as input an $\langle\cdot\rangle$-sharing, say $\langle s\rangle \mathcal{X}$ and either allows the honest parties in $\mathcal{X}$ to robustly reconstruct $s$ or ensures that the honest parties in $\mathcal{X}$ can (locally) identify at least one corrupted party in $\mathcal{X}$. The protocol is based on the following standard idea: 
let $\langle s\rangle_{i}=\left(s_{i}, u_{i}, v_{i},\left\{\mathbf{C}_{s_{j}, u_{j}, v_{j}}\right\}_{P_{j} \in \mathcal{X}}\right)$ be the information available to party $P_{i} \in \mathcal{X}$ corresponding to $\langle s\rangle_{\mathcal{X}}$. Then each $P_{i}$ broadcasts $s_{i}, u_{i}, v_{i}$ to the parties in $\mathcal{X}$ via $\mathcal{F}_{\mathrm{BC}}$. Let each party $P_{i}$ receive $\bar{s}_{j}, \bar{u}_{j}, \bar{v}_{j}$ from $P_{j}$. $P_{i}$ then verifies if $\mathbf{C}_{s_{j}, u_{j}, v_{j}}=\operatorname{Comm}_{\mathrm{ck}}\left(\bar{s}_{j} ; \bar{u}_{j}, \bar{v}_{j}\right)$. If the verification fails, $P_{i}$ identifies $P_{j}$ to be corrupted and outputs (Failure, $i, j$ ); otherwise $P_{i}$ sums up all the shares to obtain $s$ and outputs (Success, $i, s$ ). In the rest of the description, we will say that the parties in $\mathcal{X}$ participate in $\Pi_{\operatorname{REC}\langle\cdot\rangle}$ with $\langle s\rangle_{\mathcal{X}}$ and each $P_{i} \in \mathcal{X}$ outputs either (Success, $\left.i, s\right)$ or (Failure, $i, j$ ) to mean the above. The protocol has communication complexity $\mathcal{B C}(|\mathcal{X}| \kappa,|\mathcal{X}|)$.

Beaver's Multiplication Protocol. Protocol $\Pi_{\mathrm{BEA}}\left(\langle x\rangle_{\mathcal{X}},\langle y\rangle_{\mathcal{X}},\langle a\rangle_{\mathcal{X}},\langle b\rangle_{\mathcal{X}},\langle c\rangle_{\mathcal{X}}\right)$ is a standard protocol for securely computing $\langle x \cdot y\rangle_{\mathcal{X}}$ from $\langle x\rangle_{\mathcal{X}}$ and $\langle y\rangle_{\mathcal{X}}$, at the cost of two public reconstruction. The protocol assumes that the parties in $\mathcal{X} \subset \mathcal{P}$ have access to an $\langle\cdot\rangle_{\mathcal{X}}$-shared random multiplication triple $(a, b, c)$ unknown to the adversary, with $c=a \cdot b$. The protocol is based on the principle that $x \cdot y=(x-a+a) \cdot(y-$ $b+b)=d e+d b+a e+c$, where $d=(x-a)$ and $e=(y-b)$. Hence if the parties in $\mathcal{X}$ reconstruct $d$ and $e$, then they can locally compute $\langle x \cdot y\rangle_{\mathcal{X}}=d e+d \cdot\langle b\rangle_{\mathcal{X}}+e \cdot\langle a\rangle_{\mathcal{X}}+\langle c\rangle_{\mathcal{X}}$. The security of $x$ and $y$ follows even after the reconstruction of $d$ and $e$, as $x$ and $y$ are masked by random and private $a$ and $b$ respectively. To reconstruct $d$ and $e$, the parties in $\mathcal{X}$ first locally compute $\langle d\rangle_{\mathcal{X}}=\langle x-a\rangle_{\mathcal{X}}$ and $\langle e\rangle_{\mathcal{X}}=\langle y-b\rangle_{\mathcal{X}}$, followed by invoking $\Pi_{\operatorname{REC}\langle\cdot\rangle}$ with inputs $\langle d\rangle_{\mathcal{X}}$ and $\langle e\rangle_{\mathcal{X}}$. Depending on whether the instances of $\Pi_{\operatorname{REC}\langle\cdot\rangle}$ are successful or not, an honest party in $\mathcal{X}$ may output (Success, $i,\langle x \cdot y\rangle_{i}$ ) or (Failure, $i, j$ ). In the rest of the description, we will say that the parties in $\mathcal{X}$ participate in $\Pi_{\mathrm{BEA}}\left(\langle x\rangle_{\mathcal{X}},\langle y\rangle_{\mathcal{X}},\langle a\rangle_{\mathcal{X}},\langle b\rangle_{\mathcal{X}},\langle c\rangle_{\mathcal{X}}\right)$ and each $P_{i}$ output either (Success, $i,\langle x \cdot y\rangle_{i}$ ) or (Failure, $i, j$ ) to mean the above. The protocol has communication complexity $\mathcal{B C}(|\mathcal{X}| \kappa,|\mathcal{X}|)$.

\section{D.1 The Preparation Stage of Protocol $\Pi_{\mathrm{C}}^{\mathrm{NR}}$}

We are now ready to discuss the preparation stage of our protocol $\Pi_{\mathrm{C}}^{\mathrm{NR}}$. We pursue the same outline as followed by the preparation stage of the MPC protocol of [12] and describe the same briefly below. This is followed by the required adaptations in our context. The preparation stage of [12] provides security with abort. Namely the protocol generates the required $\langle\cdot\rangle$-shared triplets if all the parties behave honestly; otherwise if the honest parties identify any wrong-doing then they simply abort.

- Triple Generation: The involved parties generate many random $\langle\cdot\rangle$-shared triplets by executing many instances of $\Pi_{\text {TRIPLE }}$, assuming no active corruptions.

- Verification of the Triples via Cut-and-choose: A random fraction of the triplets are verified via cutand-choose to detect any cheating attempts. Specifically, a random subset of generated triplets are selected and the parties are asked to disclose the randomness that they used in the instances of $\Pi_{\mathrm{TRIPLE}}$ for generating the selected triplets. If any cheating is detected then the involved parties abort, otherwise they proceed to the next step. If the test passes then with high probability it is ensured that the majority of the remaining untested triplets are "good" in the sense that they are honestly generated.

- Proof of Knowledge: The goal of this test is to ensure that for each remaining triplet, every party has the knowledge of their shares, thus ensuring independence required for UC security. More specifically, during the generation of an untested triplet, a corrupted party $P_{i}$ could broadcast an arbitrary $\mathbf{C}_{a_{i, \star}, \star}, \mathbf{C}_{b_{i}, \star, \star}$ or $\mathbf{C}_{c_{i}, \star,}$, being oblivious to $a_{i}, b_{i}$ and $c_{i}$. This is prevented by the following steps: First parties generate random $\langle\cdot\rangle$-shared values (by calling $\mathcal{F}_{\operatorname{GENRAND}\langle\cdot\rangle}$ ) and then they open the difference of the triplets and those random shared values via protocol $\Pi_{\operatorname{REC}\langle\cdot\rangle}$. Opening these differences is indeed a very simple proof of knowledge (see [12]). A cheating is detected if some of the opening fail. In that case the involved parties abort, otherwise they proceed to the next step. 
- Verification of the Triplets via Sacrificing Trick: At this stage, the remaining triplets are verified for correctness via the well-known "sacrificing" trick [13]. Namely for every pair of remaining shared triplets $(a, b, c)$ and $(x, y, z)$, the parties generate a random $r$ and recompute an $\langle\cdot\rangle$-sharing of $a \cdot b$, by assuming $r x, r y, r^{2} z$ as a multiplication triplet; protocol $\Pi_{\mathrm{BEA}}$ is used for the same. Ideally if $(a, b, c)$ and $(x, y, z)$ are multiplication triplets, then the difference of the sharing of $c$ and the recomputed $a b$ should be a sharing of zero, which is verified by the parties publicly (using protocol $\Pi_{\operatorname{ReC}\langle\cdot\rangle}$ ). If any cheating is detected then the parties abort, else they proceed to the next step after discarding $(x, y, z)$, whose security is sacrificed during the verification of $(a, b, c)$. It follows that if the test passes then except with probability $1 / p$ over the choice of $r$, the triplet $(a, b, c)$ is indeed a correct multiplication triplet (see [12] for the details).

- Privacy Amplification: At this stage, the parties jointly perform privacy amplification and "distill" $C_{M}+C_{R}$ fully random private triplets from a set of $\mathcal{O}\left(\left(C_{M}+C_{R}\right)+X\right)$ triplets, where $X$ of them might not be private ${ }^{11}$; recall that $C_{M}$ and $C_{R}$ are the number of multiplication and random gates respectively in the circuit $\mathrm{C}$. For this, $\mathcal{F}_{\mathrm{GENRAND}\langle\cdot\rangle}$ along with $\Pi_{\mathrm{BEA}}$ is used. If any cheating is detected during $\Pi_{\mathrm{BEA}}$, then the parties abort.

In our context, it is not enough to abort when a wrong-doing is detected. If some party $P_{i} \in \mathcal{X}$ identifies any party $P_{j} \in \mathcal{X}$ cheating in any of the steps for preparation stage, $P_{i}$ alarms the parties in $\mathcal{P}$ by raising a complaint against $P_{j}$. This allows the parties in $\mathcal{P}$ to localize the fault to a pair of parties $\left(P_{i}, P_{j}\right)$. To simplify the fault-localization, we set a designated party $P_{\text {Ref }} \in \mathcal{X}$ with the smallest index Ref as the referee to locally identify any fault and report the same to the parties in $\mathcal{P}$. The fault localization step in each stage of the preparation stage is emphasized below.

- Fault Localization During the Verification of the Triples via Cut-and-choose: The parties in $\mathcal{X}$ first run the steps for the cut-and-choose triple-verification as in [12]. If any party $P_{i}$ locally identifies any fault then it raises an alarm for the parties in $\mathcal{P}$. On receiving the alarm, every party in $\mathcal{X}$ broadcasts (to the parties in $\mathcal{X}$ ) their entire view (including the randomness used) in the generation of the triplets under testing. The referee $P_{\text {Ref }}$ then "recomputes" every message a party $P_{i} \in \mathcal{X}$ should send to every other party $P_{j} \in \mathcal{X}$ and compares them with what $P_{i}$ claims to send and what $P_{j}$ claimed to receive. In case there is any mis-match, then $P_{\text {Ref }}$ raises a complaint against both $P_{i}$ and $P_{j}$ among $\mathcal{P}$ and urges $P_{i}$ and $P_{j}$ to respond. Now depending upon the response, the parties can localize the fault to either $\left(P_{\text {Ref }}, P_{i}\right)$ or $\left(P_{\text {Ref }}, P_{j}\right)$ or $\left(P_{i}, P_{j}\right)$. The important observation is that fault will never be localized to a pair of honest parties from $\mathcal{X}$. This is because the property of $\Pi_{\text {MULT }}$ ensures that if both the participating parties are honest then they never conflict with each other. A located pair will contain at least one corrupted party.

- Fault Localization in Proof of Knowledge: The parties in $\mathcal{X}$ execute the same steps as in [12] for proving the knowledge of their shares. If any party $P_{i} \in \mathcal{X}$ locally identifies any fault during the instances of $\Pi_{\mathrm{ReC}\langle\cdot\rangle}$ (used to open the differences of triplets and random shared values), then $P_{i}$ raises an alarm among the set $\mathcal{P}$, while the referee $P_{\text {Ref }}$ is assigned the task of publicly reporting the identity of the party $P_{j}$ it has caught cheating. The fault is then localized to $\left(P_{j}, P_{\text {Ref }}\right)$. If an honest $P_{i}$ raises an alarm, but a corrupted $P_{\text {Ref }}$ does not identify any cheater, then the fault is localized to $\left(P_{i}, P_{\text {Ref }}\right)$. It is easy to note that a located pair will contain at least one corrupted party.

- Fault Localization During the Verification of the Triplets via Sacrificing Trick: Here the parties in $\mathcal{X}$ first apply the sacrificing trick on each pair of remaining triplets. Now there are three situations under which a party $P_{i} \in \mathcal{X}$ can detect a fault. (a) The instances of $\Pi_{\mathrm{BEA}}$ is unsuccessful. In this case, the

\footnotetext{
${ }^{11}$ For the specific instantiation of $\Pi_{\text {MULT }}$ based on Paillier encryption, this is indeed the case if one of the participating parties in $\Pi_{\text {MULT }}$ is corrupted; see [12] for the details.
} 
parties in $\mathcal{P}$ localize the fault in the same way as in the previous step. Namely $P_{i}$ raises an alarm while $P_{\text {Ref }}$ is asked to identify the cheating party. (b) The instances of $\Pi_{\operatorname{REC}\langle\cdot\rangle}$ to open the difference of $a b$ and $c$ fails; the fault-localization in this case is also the same as in the previous step. (c) The difference of $a b$ and $c$ is non-zero. Clearly in this case, at least one of the involved triplet (in the pair) is not generated correctly and so the parties in $\mathcal{P}$ perform the fault-localization in the same way as in the cut-and-choose step. Namely, all the parties in $\mathcal{X}$ publicly open (to the parties in $\mathcal{X}$ ) their entire view produced during the generation of the two triplets and $P_{\text {Ref }}$ is then asked to find a pair of "conflicting" parties.

- Fault Localization in Privacy Amplification: The parties in $\mathcal{X}$ execute the steps for privacy amplification [12]. If any cheating is detected by a party $P_{i} \in \mathcal{X}$ during the involved instances of $\Pi_{\mathrm{BEA}}$, then the parties in $\mathcal{P}$ perform the fault localization in the same way as it is done during for a failed instance of $\Pi_{\mathrm{BEA}}$ in the previous stage.

The protocol steps for the preparation stage are given in Figure 17, where we give the formal steps for the fault localization with respect to only the first two phases; the formal steps for the fault localization for the remaining phases is not provided to avoid repetition.

In the protocol, $B$ and $\lambda$ are two parameters. In [12], it was shown that their preparation stage provides a statistical security of $2^{-B \log _{2}(1+\lambda)}$. They set $B$ and $\lambda$ as $B=3.6 s$ and $\lambda=1 / 4$ to achieve a statistical security of $2^{-s}$. Since our preparation stage is almost the same as that of [12] bar the fault-localization steps (which does not affect the statistical security at all), it follows easily via [12] that our preparation stage also provides a statistical security of $2^{-B \log _{2}(1+\lambda)}$. Intuitively this is due to the following reason: define a triplet to be a good one if the adversary could open it correctly during the Cut-and-choose step and make an honest party accept (this implies that such a triplet is generated honestly), otherwise call the triplet a bad triplet (i.e. such triplets are not generated honestly and so adversary may know some information about honest partys' shares for such triplets). Then it follows from [12] that if the protocol reaches the Privacy Amplification phase, then the probability that the triplets considered during this phase has more than $B$ bad (and hence non-private) triplets is at most $(1+\lambda)^{-B}$. As a result, adversary may know at most $B$ points on the polynomials $F(\cdot)$ and $G(\cdot)$ of degree at most $d$, implying $\mathrm{C}_{\mathrm{M}}+\mathrm{C}_{\mathrm{R}}$ degree of freedom in the view of the adversary. Note that as suggested in [12], instead of creating "big" polynomials $F(\cdot)$ and $G(\cdot)$ of huge degrees, we can partition the remaining triplets in $\mathcal{M}$ into batches of smaller size and accordingly use many polynomials of small degree, without affecting the security properties; we prefer to present the Privacy Amplification phase the way presented in [12]. 


\section{Preparation Stage of $\Pi_{\mathrm{C}}^{\mathrm{NR}}$}

The public input to the protocol is a set of parties $\mathcal{X} \subset \mathcal{P}$ containing at least one honest party and a referee $P_{\text {Ref }} \in \mathcal{X}$, with the smallest index Ref. For the session id sid, every party $P_{i} \in \mathcal{P}$ participates with (sid, $i$ ) and does the following:

Triple-generation Assuming No Active Corruption - If $P_{i} \in \mathcal{X}$ then participate in the protocol $\Pi_{\text {TRIPLE }}(1+\lambda)\left(4\left(C_{M}+\right.\right.$ $\left.\left.C_{R}\right)+4 B-2\right)$ times to generate a set $\mathcal{M}$ of $(1+\lambda)\left(4\left(C_{M}+C_{R}\right)+4 B-2\right)\langle\cdot\rangle$-shared triplets.

Testing the Triplets via Cut-and-Choose - If $P_{i} \in \mathcal{X}$ then do the following:

- Call $\mathcal{F}_{\text {GenRand }\langle\cdot\rangle}$ to sample a random string str that determines a subset $\mathcal{T} \subset \mathcal{M}$ of size $\lambda\left(4\left(\mathrm{C}_{\mathrm{M}}+\mathrm{C}_{\mathrm{R}}\right)+4 B-2\right)$. Set $\mathcal{M}=\mathcal{M} \backslash \mathcal{T}$. Let $\operatorname{View}_{i}^{\mathcal{T}}$ denote the randomness used by $P_{i}$ and the messages received from the other parties in $\mathcal{X}$, during the instances of $\Pi_{\mathrm{TRIPLE}}$ used for generating the triplets in $\mathcal{T}$. Reveal $\mathrm{View}_{i}^{\mathcal{T}}$ to the parties in $\mathcal{X}$ by calling $\mathcal{F}_{\mathrm{BC}}$ with $\left(\mathrm{sid}, i, \operatorname{View}_{i}^{\mathcal{T}}, \mathcal{X}\right)$.

- Corresponding to each $P_{j} \in \mathcal{X}$, receive (sid, $i, j$, View $w_{j}^{\mathcal{T}}$ ) from $\mathcal{F}_{\mathrm{BC}}$. Using $\left\{\mathrm{View}_{j}^{\mathcal{T}}\right\}_{P_{j} \in \mathcal{X}}$, reproduce every message that should have been sent by every sender $P_{a} \in \mathcal{X}$ to every receiver $P_{b} \in \mathcal{X}$ during the generation of the triplets in $\mathcal{T}$, and compare it with the corresponding value that the recipient $P_{b}$ claims to have received. If any conflict is detected, then do the following for the smallest indexed conflicting parties $P_{a}, P_{b}$ :

- If $P_{i} \neq P_{\mathrm{Ref}}$, then call $\mathcal{F}_{\mathrm{BC}}$ with (sid, $\left.i, \operatorname{Err}, \mathcal{P}\right)$ to indicate to the parties in $\mathcal{P}$ that a conflict has been detected.

- Else call $\mathcal{F}_{\mathrm{BC}}$ with (sid, Ref, $\operatorname{Err}, P_{a}, P_{b}, l, x, \bar{x}, \mathcal{P}$ ) to indicate that referee $P_{i}$ identified $P_{a}, P_{b} \in \mathcal{X}$ the least indexed conflicting parties and a message with index $l$ where $P_{a}$ should have sent $x$ but $P_{b}$ claimed to receive $\bar{x} \neq x$.

- If the message (sid, $i$, Ref, Err, $P_{a}, P_{b}, l, x, \bar{x}$ ) is received from $\mathcal{F}_{\mathrm{BC}}$ and if $P_{a}=P_{i}$ or $P_{b}=P_{i}$, then call $\mathcal{F}_{\mathrm{BC}}$ with (sid, $i$, Agree, $\left.P_{\text {Ref }}, \mathcal{P}\right)$ to indicate that you agree with $P_{\text {Ref }}$, else call $\mathcal{F}_{\mathrm{BC}}$ with $\left(\right.$ sid, $i$, Disagree, $\left.P_{\text {Ref }}, \mathcal{P}\right)$.

Fault Localization -

- If the message (sid, $i$, Ref, Err, $\left.P_{a}, P_{b}, l, x, \bar{x}\right)$ is received from $\mathcal{F}_{\mathrm{BC}}$ and subsequently (a) if (sid, $i, a$, Disagree, $P_{\text {Ref }}$ ) is received from $\mathcal{F}_{\mathrm{BC}}$, then output (sid, $i$, Failure, $\left.P_{\mathrm{Ref}}, P_{a}\right)$ and halt (b) if (sid, $i, b$, Disagree, $P_{\mathrm{Ref}}$ ) is received from $\mathcal{F}_{\mathrm{BC}}$, then output (sid, $i$, Failure, $P_{\mathrm{Ref}}, P_{b}$ ) and halt. Else output (sid, $i$, Failure, $P_{a}, P_{b}$ ) and halt.

- If no message of the form (sid, $i$, Ref, Err, $\star, \star, \star, \star, \star)$ is received from $\mathcal{F}_{\mathrm{BC}}$, but corresponding to some $P_{j} \in \mathcal{X}$ the message (sid, $i, j, \operatorname{Err}$ ) is received from $\mathcal{F}_{\mathrm{BC}}$, then output (sid, $i$, Failure, $P_{\mathrm{Ref}}, P_{j}$ ) and halt.

Proof of Knowledge - If $P_{i} \in \mathcal{X}$ then do the following for every (untested) triplet $\left(\langle a\rangle_{\mathcal{X}},\langle b\rangle_{\mathcal{X}},\langle c\rangle_{\mathcal{X}}\right)$ in $\mathcal{M}$ : Sample three random $\langle\cdot\rangle$-shared values $\langle r\rangle_{\mathcal{X}},\langle s\rangle_{\mathcal{X}},\langle u\rangle_{\mathcal{X}}$ by invoking $\mathcal{F}_{\mathrm{GeNRAND}\langle\cdot\rangle}$. Participate in instances of $\Pi_{\operatorname{Rec}\langle\cdot\rangle}$ with $\langle r-$ $a\rangle_{\mathcal{X}},\langle s-b\rangle_{\mathcal{X}}$ and $\langle u-c\rangle_{\mathcal{X}}$. If (sid, Failure, $\left.i, j\right)$ is the output in any of the instances of $\Pi_{\operatorname{ReC}\langle\cdot\rangle}$, then do the following:

- If $P_{i} \neq P_{\text {Ref }}$ then call $\mathcal{F}_{\mathrm{BC}}$ with $(\operatorname{sid}, i, j, \operatorname{Err}, \mathcal{P})$ to indicate that a cheating has been detected.

- Else if $P_{i}=P_{\text {Ref }}$ then call $\mathcal{F}_{\mathrm{BC}}$ with (sid, Ref, Err, $j, \mathcal{P}$ ) to indicate $P_{j}$ is identified as a cheater; if there are several such $P_{j}$ s then select the one with the minimum index $j$.

Fault Localization - If a message $(\operatorname{sid}, i$, Ref, $\operatorname{Err}, j, \mathcal{P})$ is received from $\mathcal{F}_{\mathrm{BC}}$, then output (sid, $i$, Failure, $\left.P_{\mathrm{Ref}}, P_{j}\right)$ and halt. Else if a message (sid, $i, j, \operatorname{Err})$ is received from $\mathcal{F}_{\mathrm{BC}}$, then output (sid, $i$, Failure, $\left.P_{i}, P_{j}\right)$ and halt.

Verification Via sacrificing Trick - If $P_{i} \in \mathcal{X}$ then do the following for every pair of triplets $\left(\langle a\rangle_{\mathcal{X}},\langle b\rangle_{\mathcal{X}},\langle c\rangle_{\mathcal{X}}\right)$ and $\left(\langle x\rangle_{\mathcal{X}},\langle y\rangle_{\mathcal{X}},\langle z\rangle_{\mathcal{X}}\right)$ in $\mathcal{M}$ : Call $\mathcal{F}_{\mathrm{GenRAnd}}\langle\cdot\rangle$ and sample a random ${ }^{a} r$. Participate in $\Pi_{\mathrm{BEA}}\left(\langle a\rangle_{\mathcal{X}},\langle b\rangle_{\mathcal{X}},\langle r x\rangle_{\mathcal{X}},\langle r y\rangle_{\mathcal{X}},\left\langle r^{2} z\right\rangle_{\mathcal{X}}\right)$ for computing $\langle\bar{c}\rangle_{\mathcal{X}}$ followed by participation in $\Pi_{\mathrm{REC}\langle\cdot\rangle}$ with $\langle\bar{c}-c\rangle_{\mathcal{X}}$. If no cheating has been identified during $\Pi_{\mathrm{BEA}}, \Pi_{\mathrm{REC}\langle\cdot\rangle}$ and if $\bar{c}-c=0$, then store $\left(\langle a\rangle_{\mathcal{X}},\langle b\rangle_{\mathcal{X}},\langle c\rangle_{\mathcal{X}}\right)$ for future use and drop $\left(\langle x\rangle_{\mathcal{X}},\langle y\rangle_{\mathcal{X}},\langle z\rangle_{\mathcal{X}}\right)$ from $\mathcal{M}$. Else proceed to the fault-localization step.

Fault Localization - If the parties in $\mathcal{X}$ have raised a complaint due to the failure of $\Pi_{\mathrm{BEA}}$ or $\Pi_{\mathrm{REC}\langle\cdot\rangle}$, then localize the fault in the same way as in the case of fault-localization for the Proof of Knowledge step. Else localize the fault in the same way as in the Cut-and-Choose step by asking the parties in $\mathcal{X}$ to open their entire view of the disputed triplet.

Privacy Amplification - The parties in $\mathcal{X}$ are now left with $2\left(C_{M}+C_{R}\right)+2 B-1$ triplets $\left\{\left(\left\langle a^{k}\right\rangle_{\mathcal{X}},\left\langle b^{k}\right\rangle_{\mathcal{X}}\right.\right.$, $\left.\left.\left\langle c^{k}\right\rangle_{\mathcal{X}}\right)\right\}_{k=1, \ldots, 2\left(\mathrm{C}_{\mathrm{M}}+\mathrm{C}_{\mathrm{R}}\right)+2 B-1}$ in $\mathcal{M}$. Let $d=\left(\mathrm{C}_{\mathrm{M}}+\mathrm{C}_{\mathrm{R}}\right)+B-1$. If $P_{i} \in \mathcal{X}$ then do the following:

- Invoke $\mathcal{F}_{\text {GenRand }\langle\cdot\rangle} 2(d+1)$ times to generate $\left\langle f^{(1)}\right\rangle_{\mathcal{X}}, \ldots,\left\langle f^{(d+1)}\right\rangle_{\mathcal{X}}$ and $\left\langle g^{(1)}\right\rangle_{\mathcal{X}}, \ldots,\left\langle g^{(d+1)}\right\rangle_{\mathcal{X}}$.

- Let $F(\cdot)$ and $G(\cdot)$ be the polynomials of degree at most $d$ such that $F\left(\alpha_{k}\right)=f^{(k)}$ and $G\left(\alpha_{k}\right)=g^{(k)}$ for $k=$ $1, \ldots, d+1$. Locally compute $\left\langle F\left(\alpha_{d+2}\right)\right\rangle_{\mathcal{X}}, \ldots,\left\langle F\left(\alpha_{2 d+1}\right)\right\rangle_{\mathcal{X}}$ and $\left\langle G\left(\alpha_{d+2}\right)\right\rangle_{\mathcal{X}}, \ldots,\left\langle G\left(\alpha_{2 d+1}\right)\right\rangle_{\mathcal{X}}$. For $k=$ $1, \ldots, 2 d+1$, participate in $\Pi_{\mathrm{BEA}}\left(\left\langle F\left(\alpha_{k}\right)\right\rangle_{\mathcal{X}},\left\langle G\left(\alpha_{k}\right)\right\rangle_{\mathcal{X}},\left\langle a^{(k)}\right\rangle_{\mathcal{X}},\left\langle b^{(k)}\right\rangle_{\mathcal{X}},\left\langle c^{(k)}\right\rangle_{\mathcal{X}}\right)$ for computing $\left\langle h^{(k)}\right\rangle_{\mathcal{X}}=$ $\left\langle F\left(\alpha_{k}\right) \cdot G\left(\alpha_{k}\right)\right\rangle_{\mathcal{X}}$.

- If any cheating is identified during $\Pi_{\mathrm{BEA}}$, then proceed to the fault localization step. Else let $H(\cdot)$ be the polynomial of degree at most $2 d$ such that $H\left(\alpha_{i}\right)=h^{(i)}$ for $i=1, \ldots, 2 d+1$. Then output (sid, $i$, Success, $\left\{\left(\left\langle\mathbf{a}^{(k)}\right\rangle_{\mathcal{X}},\left\langle\mathbf{b}^{(k)}\right\rangle_{\mathcal{X}},\left\langle\mathbf{c}^{(k)}\right\rangle_{\mathcal{X}}\right)\right\}_{\left.k=1, \ldots, \mathrm{C}_{\mathrm{M}}+\mathrm{C}_{\mathrm{R}}\right)}$ and halt, where $\mathbf{a}^{(k)}=F\left(-\alpha_{k}\right), \mathbf{b}^{(k)}=G\left(-\alpha_{k}\right)$ and $\mathbf{c}^{(k)}=$ $H\left(-\alpha_{k}\right)$.

Fault Localization - If any complaint is raised due to the failure of $\Pi_{\mathrm{BEA}}$, then localize the fault as in the Proof of Knowledge step. Else every $P_{i} \in \mathcal{P} \backslash \mathcal{X}$ output (sid, $i$, Success) and halt.

${ }^{a}$ It is enough to sample a single $r$ for all the pairs of availabłe triplets.

Fig. 17. Generating $C_{M}+C_{R}\langle\cdot\rangle$-shared Multiplication Triples with Statistical Security $2^{-B \log _{2}(1+\lambda)}$. 


\section{D.2 Protocol $\Pi_{\mathrm{C}}^{\mathrm{NR}}$}

In this section the protocol $\Pi_{\mathrm{C}}^{\mathrm{NR}}$ is presented in Figure 18, where during the circuit-evaluation stage, we follow the idea outlined earlier in section 3.2. Note that during the circuit evaluation, an instance of $\Pi_{\mathrm{BEA}}$ may fail, in which case the parties in $\mathcal{P}$ localize the fault via the referee $P_{\text {Ref }}$ in the same way as it was done in the preparation stage.

\section{Protocol $\Pi_{\mathrm{C}}^{\mathrm{NR}}$}

The public input to the protocol is a set of parties $\mathcal{X} \subset \mathcal{P}$ containing at least one honest party and an arithmetic circuit $\mathrm{C}$ over $\mathbb{F}_{p}$ consisting of in input gates, out output gates, $C_{M}$ multiplication gates and $C_{R}$ random gates. In addition, $\left\langle x_{1}\right\rangle \mathcal{X}, \ldots,\left\langle x_{\text {in }}\right\rangle \mathcal{X}$ are the $\langle\cdot\rangle$-shared inputs for $\mathrm{C}$. Let $P_{\mathrm{Ref}} \in \mathcal{X}$ be the party with the smallest index Ref who is set as the referee to localize any fault occurred during the protocol.

For the session id sid, party $P_{i} \in \mathcal{P}$ participates with (sid, $i$ ) and does the following:

Preparation Stage: execute the steps of Figure 17.

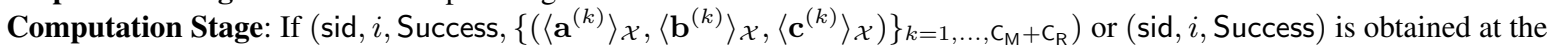
end of preparation stage, then do the following:

- $\langle\cdot\rangle$-shared Evaluation of the Circuit $\mathrm{C}$ - If $P_{i} \in \mathcal{X}$ then do the following for every gate in the circuit $\mathrm{C}$ :

- Input Gate: For $l=1, \ldots$, in, associate $\left\langle x_{l}\right\rangle_{\mathcal{X}}$ with the corresponding input gate of $\mathrm{C}$.

- Random Gate: For the $k^{t h}$ random gate in $C$ where $k \in\left\{1, \ldots, C_{\mathrm{R}}\right\}$, associate $\left\langle\mathbf{a}^{(k)}\right\rangle_{\mathcal{X}}$ as the output of the random gate.

- Addition Gate: If $\langle x\rangle_{\mathcal{X}}$ and $\langle y\rangle_{\mathcal{X}}$ are the $\langle\cdot\rangle$-shared inputs of the gate, then locally compute $\langle x+y\rangle_{\mathcal{X}}=$ $\langle x\rangle_{\mathcal{X}}+\langle y\rangle_{\mathcal{X}}$ and associate it as the output of the addition gate.

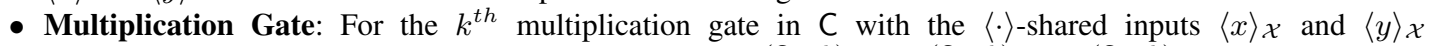
where $k \in\left\{1, \ldots, \mathrm{C}_{\mathrm{M}}\right\}$, associate the triplet $\left(\left\langle\mathbf{a}^{\left(\mathrm{C}_{\mathrm{R}}+k\right)}\right\rangle_{\mathcal{X}},\left\langle\mathbf{b}^{\left(\mathrm{C}_{\mathrm{R}}+k\right)}\right\rangle_{\mathcal{X}},\left\langle\mathbf{c}^{\left(\mathrm{C}_{\mathrm{R}}+k\right)}\right\rangle_{\mathcal{X}}\right)$. Participate in $\Pi_{\mathrm{BEA}}\left(\langle x\rangle_{\mathcal{X}},\langle y\rangle_{\mathcal{X}},\left\langle\mathbf{a}^{\left(\mathrm{C}_{\mathrm{R}}+k\right)}\right\rangle_{\mathcal{X}},\left\langle\mathbf{b}^{\left(\mathrm{C}_{\mathrm{R}}+k\right)}\right\rangle_{\mathcal{X}},\left\langle\mathbf{c}^{\left(\mathrm{C}_{\mathrm{R}}+k\right)}\right\rangle_{\mathcal{X}}\right)$ to compute $\langle x \cdot y\rangle_{\mathcal{X}}$. If (sid, $i$, Failure, $j$ ) with $P_{j} \in$ $\mathcal{X}$ is obtained during the instance of $\Pi_{\mathrm{BEA}}$ then do the following:

* If $P_{i} \neq P_{\text {Ref }}$ then call $\mathcal{F}_{\mathrm{BC}}$ with $(\operatorname{sid}, i, \operatorname{Err}, \mathcal{P}$ ) to indicate that a cheating has been detected while executing $\Pi_{\mathrm{BEA}}$.

* Else if $P_{i}=P_{\text {Ref }}$ then call $\mathcal{F}_{\mathrm{BC}}$ with (sid, Ref, Err, $\left.j, \mathcal{P}\right)$ to indicate that $P_{j}$ is identified as a cheater while executing $\Pi_{\mathrm{BEA}}$; if there are several such $P_{j}$ s then select the one with the minimum index $j$.

- Fault Localization -

- If there exists a multiplication gate in $C$ corresponding to which a message (sid, $i, \operatorname{Ref}, \operatorname{Er} r, j$ ) is received on the behalf of $P_{\text {Ref }}$ from $\mathcal{F}_{\mathrm{BC}}$ then output (sid, $i$, Failure, $P_{\mathrm{Ref}}, P_{j}$ ) and halt.

- Else if there exists a multiplication gate in C corresponding to which a message (sid, $i, j$, Err) is received from $\mathcal{F}_{\mathrm{BC}}$ on the behalf of $P_{j} \in \mathcal{X}$, but no message of the form (sid, $i$, Ref, Err, $\left.\star, \star\right)$ is received from $\mathcal{F}_{\mathrm{BC}}$ on the behalf of $P_{\text {Ref }}$, then output (sid, $i$, Failure, $P_{\text {Ref }}, P_{j}$ ) and halt.

- Else if $P_{i} \in \mathcal{P} \backslash \mathcal{X}$ then output (sid, $i$, Success) and halt; otherwise output (sid, $i$, Success, $\left.\left\langle y_{1}\right\rangle_{\mathcal{X}}, \ldots,\left\langle y_{\text {out }}\right\rangle_{\mathcal{X}}\right)$ and halt, where $\left\langle y_{1}\right\rangle_{\mathcal{X}}, \ldots,\left\langle y_{\text {out }}\right\rangle_{\mathcal{X}}$ are the $\langle\cdot\rangle$-shared outputs associated with the output gates of $\mathrm{C}$.

Fig. 18. Protocol for Secure $\langle\cdot\rangle$-shared Evaluation of a Given Circuit $C$ with Statistical Security $1-2^{-B \log _{2}(1+\lambda)}$.

The correctness of the protocol follows via the binding property of the commitment and the detailed informal discussion above, while we appeal to [12] for the proof of privacy in UC secure framework. We now prove Lemma 4 (the lemma statement is available in Section 3), by setting $\lambda=1 / 4$ and $B=3.6 s$ as done in [12], so that the protocol provides a statistical security of $2^{-s}$.

Proof of Lemma 4: We prove the communication complexity of the preparation stage, with the observation that $C_{M}+C_{R}=\mathcal{O}(|C|)$. During the Triple-generation phase, $\mathcal{O}\left(C_{M}+C_{R}+B\right)$ instances of $\Pi_{\mathrm{TRIPLE}}$ are executed by the parties in $\mathcal{X}$, thus requiring communication complexity of $\mathcal{O}\left(|\mathcal{X}|^{2}(|\mathrm{C}|+B) \kappa\right)$ and $\mathcal{B C}(|\mathcal{X}|(|C|+B) \kappa,|\mathcal{X}|)$. 
During the Cut-and-Choose phase, $\mathcal{O}\left(\mathrm{C}_{\mathrm{M}}+\mathrm{C}_{\mathrm{R}}+B\right)$ calls to $\mathcal{F}_{\text {GenRAND }\langle\cdot\rangle}$ are made for generating $\mathcal{O}\left(\mathrm{C}_{\mathrm{M}}+\mathrm{C}_{\mathrm{R}}+B\right)$ random $\langle\cdot\rangle$-shared commitments with statistical security $2^{-B \log _{2}(1+\lambda)}$, incurring communication complexity of $\mathcal{B C}(|\mathcal{X}|(|\mathrm{C}|+B) \kappa,|\mathcal{X}|)$. In addition, the parties in $\mathcal{X}$ need to broadcast among themselves their entire view of $\Pi_{\text {TRIPLE }}$ with respect to $\mathcal{O}\left(C_{M}+C_{R}+B\right)$ triplets. This incurs a communication complexity of $\mathcal{B C}\left(|\mathcal{X}|^{2}(|\mathrm{C}|+B) \kappa,|\mathcal{X}|\right)$. During the fault-localization step, the parties in $\mathcal{X}$ need to broadcast $\mathcal{O}(\kappa)$ bits to the parties in $\mathcal{P}$, thus requiring communication complexity of $\mathcal{B C}(|\mathcal{X}| \kappa, n)$.

During the Proof of Knowledge phase, $\mathcal{O}\left(\mathrm{C}_{\mathrm{M}}+\mathrm{C}_{\mathrm{R}}+B\right)$ calls to $\mathcal{F}_{\text {GENRAND }\langle\cdot\rangle}$ are made and $\mathcal{O}\left(\mathrm{C}_{\mathrm{M}}+\right.$ $\left.\mathrm{C}_{\mathrm{R}}+B\right)$ instances of $\Pi_{\mathrm{REC}\langle\cdot\rangle}$ are executed by the parties in $\mathcal{X}$, thus requiring a communication complexity of $\mathcal{B C}(|\mathcal{X}|(|C|+B) \kappa,|\mathcal{X}|)$. In addition, during the fault-localization step, the parties in $\mathcal{X}$ need to broadcast $\mathcal{O}(\kappa)$ bits to the parties in $\mathcal{P}$, thus requiring communication complexity of $\mathcal{B C}(|\mathcal{X}| \kappa, n)$.

During the Correctness phase, $\mathcal{O}\left(\mathrm{C}_{\mathrm{M}}+\mathrm{C}_{\mathrm{R}}+B\right)$ instances of $\Pi_{\mathrm{BEA}}$ and $\Pi_{\mathrm{REC}\langle\cdot\rangle}$ are executed by the parties in $\mathcal{X}$. In addition, the parties in $\mathcal{X}$ may need to publicly open among themselves the entire view of $\Pi_{\text {TRIPLE }}$ with respect to a disputed pair of triplet. Thus this phase has communication complexity of $\mathcal{B C}(|\mathcal{X}|(|\mathrm{C}|+B) \kappa,|\mathcal{X}|)$, with an additional communication complexity of $\mathcal{B C}(|\mathcal{X}| \kappa, n)$ for the faultlocalization step. It follows easily that the Privacy Amplification phase as well as the circuit evaluation stage has communication complexity of $\mathcal{B C}(|\mathcal{X}|(|\mathrm{C}|+B) \kappa,|\mathcal{X}|)$ for executing the steps within $\mathcal{X}$ and has communication complexity of $\mathcal{B C}(|\mathcal{X}| \kappa, n)$ for any possible fault-localization.

During the computation stage, $C_{\mathrm{M}}$ instances of $\Pi_{\mathrm{BEA}}$ are executed and fault-localization is done at most once. It thus follows that setting $B=3.6 s$, the protocol has communication complexity $\mathcal{O}\left(|\mathcal{X}|^{2}(|\mathrm{C}|+\right.$ $s) \kappa), \mathcal{B C}\left(|\mathcal{X}|^{2}(|\mathrm{C}|+s) \kappa,|\mathcal{X}|\right)$ and $\mathcal{B C}(|\mathcal{X}| \kappa, n)$.

\section{E Proof of Theorem 1}

Security. We prove the security by designing a simulator for the protocol $\Pi_{f}$. Let $T \subset \mathcal{P}$ be the set of parties under the control of $\mathcal{A}$ during the protocol $\Pi_{f}$; we present a simulator $\mathcal{S}_{f}$ (interacting with the functionality $\mathcal{F}_{f}$ ) for $\mathcal{A}$ in Figure 19. The high level idea for the simulator is the following: the simulator takes the input $\left\{x^{(i)}\right\}_{P_{i} \in T}$ and interacts with $\mathcal{F}_{f}$ to obtain the function output $y$. The simulator then invokes $\mathcal{A}$ with the inputs $\left\{x^{(i)}\right\}_{P_{i} \in T}$ and simulates each message that $\mathcal{A}$ would have received in the protocol $\Pi_{f}$ from the honest parties and from the functionalities called therein, step by step. Notice that the simulator $\mathcal{S}_{f}$ also needs to simulate the protocol steps of the honest parties for the sub-protocols $\Pi_{[\cdot] \rightarrow\langle\cdot\rangle}$, $\Pi_{\langle\cdot\rangle \rightarrow[\cdot]}, \Pi_{\mathrm{ckt}_{l}}^{\mathrm{NR}}$ and $\Pi_{\mathrm{RANDZERO}[\cdot] \cdot}$. Specifying the simulator steps for these subprotocols would make the description of $\mathcal{S}_{f}$ complicated. So for the ease of presentation, we define three sub-simulators $\mathcal{S}_{[\cdot] \rightarrow\langle\cdot\rangle}$ (Fig. 20), $\mathcal{S}_{\langle\cdot\rangle \rightarrow[\cdot]}$ (Fig. 21), and $\mathcal{S}_{\text {RandZero[.] }}$ (Fig. 22) which are invoked by $\mathcal{S}_{f}$ for simulating the steps of the honest parties for the instances of $\Pi_{[\cdot] \rightarrow\langle\cdot\rangle}, \Pi_{\langle\cdot\rangle \rightarrow[\cdot]}$ and $\Pi_{\text {RANDZERo[.] }}$ respectively; technically, the steps specified for $\mathcal{S}_{[\cdot] \rightarrow\langle\cdot\rangle}, \mathcal{S}_{\langle\cdot\rangle \rightarrow[\cdot]}$ and $\mathcal{S}_{\text {RANDZERo[.] }}$ are actually done by the main simulator $\mathcal{S}_{f}$. While invoking these "subsimulators", $\mathcal{S}_{f}$ will provide its entire internal state to them and the sub-simulators then return back their internal state (after the required simulation) to the main simulator. Similarly, we also assume the presence of a simulator $\mathcal{S}_{\mathrm{ckt}_{l}}^{\mathrm{NR}}$, which can be invoked by $\mathcal{S}_{f}$ to simulate the steps of the honest parties for the protocol $\mathcal{S}_{\mathrm{ckt}_{l}}^{\mathrm{NR}}$. We do not explicitly give the steps of $\mathcal{S}_{\mathrm{ckt}}^{\mathrm{NR}}$, but rather appeal to the simulator of the MPC protocol of [12] because the protocol steps of $\Pi_{\mathrm{ckt}_{l}}^{\mathrm{NR}}$ are almost the same as the MPC protocol of [12], bar the faultlocalization steps. However, simulating the steps of fault-localization is straight forward, since the simulator will know the entire states of all the honest parties in $\Pi_{\mathrm{ckt}_{l}}^{\mathrm{NR}}$ and so any wrong-doings by the corrupted parties can be easily identified by the simulator exactly as it was identified by an honest party in $\Pi_{\mathrm{ckt}_{l}}^{\mathrm{NR}}$.

It is easy to show that $\operatorname{IDEAL}_{\mathcal{F}_{f}, \mathcal{S}_{f}, \mathcal{Z}} \approx \operatorname{REAL}_{\Pi_{f}, \mathcal{A}, \mathcal{Z}}$ in the $\left(\mathcal{F}_{\mathrm{CRS}}, \mathcal{F}_{\mathrm{BC}}, \mathcal{F}_{\text {CommitteE }}, \mathcal{F}_{\mathrm{Gen}[\cdot]}, \mathcal{F}_{\mathrm{GenRAND}\langle\cdot\rangle}\right.$, $\mathcal{F}_{\text {ZK.BC}}$ )-hybrid settings due to the privacy of the the secret sharing schemes and the statistical hiding prop- 


\section{Simulator $\mathcal{S}_{f}$}

The simulator plays the role of the honest parties and simulates each step of the protocol $\Pi_{f}$ as follows. The communication of the $\mathcal{Z}$ with the adversary $\mathcal{A}$ is handled as follows: Every input value received by the simulator from $\mathcal{Z}$ is written on $\mathcal{A}$ 's input tape. Likewise, every output value written by $\mathcal{A}$ on its output tape is copied to the simulator's output tape (to be read by the environment $\mathcal{Z}$ ). The simulator then does the following for the session ID sid:

Initialization. $\mathcal{S}_{f}$ sets its internal variables $\mathcal{L}=\mathcal{P}, \mathfrak{n}=n, \mathfrak{t}=t$ and NewCom $=1$.

CRS Generation. On receiving (sid, $i$ ) from every $P_{i} \in T$, simulator $\mathcal{S}_{f}$, on behalf of $\mathcal{F}_{\text {CRs }}$, computes Gen $\left(1^{\kappa}\right) \rightarrow$ $\left(\mathrm{ck}, \tau_{0}, \tau_{1}\right)$ and $G\left(1^{\kappa}\right) \rightarrow(\mathrm{pk}, \mathrm{sk})$, sets CRS $=(\mathrm{ck}, \mathrm{pk})$ and sends (sid, $\left.i, C R S\right)$ to every $P_{i} \in T$.

Input commitment. On behalf of every honest party $P_{i} \in \mathcal{P} \backslash T, \mathcal{S}_{f}$ picks three random polynomials over $\mathbb{F}_{p}, f^{(i)}(\cdot), g^{(i)}(\cdot), h^{(i)}(\cdot)$ of degree $t$ such that $f^{(i)}(0)=0$ and imitates the behavior of the honest parties. That is, $\mathcal{S}_{f}$ computes the commitment $\mathbf{C}_{f^{(i)}(0), g^{(i)}(0), h^{(i)}(0)}=\operatorname{Comm}_{\mathrm{ck}}\left(f^{(i)}(0) ; g^{(i)}(0), h^{(i)}(0)\right)$ and sends (sid, $\left.i, \mathbf{C}_{f^{(i)}(0), g^{(i)}(0), h^{(i)}(0)}\right)$ to every corrupted $P_{j} \in T$ on behalf of $\mathcal{F}_{\mathrm{BC}}$. When a corrupted $P_{i} \in T$ invokes $\mathcal{F}_{\mathrm{BC}}$ with (sid, $\left.i, \mathbf{C}_{f^{(i)}(0), g^{(i)}(0), h^{(i)}(0)}, \mathcal{P}\right)$, simulator $\mathcal{S}_{f}$ acts on behalf of $\mathcal{F}_{\mathrm{BC}}$ and sends $\mathbf{C}_{f^{(i)}(0), g^{(i)}(0), h^{(i)}(0)}$ to every $P_{j} \in T$.

[·]-sharing of Inputs. For every honest $P_{i} \in \mathcal{P} \backslash T$, simulator $\mathcal{S}_{f}$ acts on behalf of functionality $\mathcal{F}_{\mathrm{GeN}[\cdot]}$ with (sid, $\left.i, f^{(i)}(\cdot), g^{(i)}(\cdot), h^{(i)}(\cdot)\right)$ and hands (sid, $\left.j, i,\left[f^{(i)}(0)\right]_{j}\right)$ to every $P_{j} \in T$. Then for every corrupted $P_{i} \in T$, on receiving (sid, $\left.i, f^{(i)}(\cdot), g^{(i)}(\cdot), h^{(i)}(\cdot)\right)$ from $P_{i}$ (as the dealer), $\mathcal{S}_{f}$, on behalf of $\mathcal{F}_{\text {Gen }[\cdot]}$, sends $\left(\operatorname{sid}, j, i,\left[f^{(i)}(0)\right]_{i}\right)$ to every $P_{j} \in T$, after verifying the polynomials $f^{(i)}(\cdot), g^{(i)}(\cdot), h^{(i)}(\cdot)$ with respect to the corresponding commitment $\mathbf{C}_{f^{(i)}(0), g^{(i)}(0), h^{(i)}(0)}$ (as done by the functionality $\left.\mathcal{F}_{\mathrm{GEN}[\cdot]}\right)$. Locally, simulator maintains the following information:

- $\mathcal{S}_{f}$ stores the input of corrupted $P_{i} \in T$ as $x^{(i)}=f^{(i)}(0)$, where $f^{(i)}(\cdot)$ is received from corrupted $P_{i}$. Further it sets the input of honest $P_{i} \in \mathcal{P} \backslash T$ as $x^{(i)}=0$.

- For every $P_{i} \in \mathcal{P}$, it stores the entire $\left[x^{(i)}\right]$.

$\mathcal{S}_{f}$ hands $\left\{x^{(i)}\right\}_{P_{i} \in T}$ to the MPC functionality $\mathcal{F}_{f}$ on behalf of the corrupted parties and gets back the outputs $y$ from the functionality. Next $\mathcal{S}_{f}$ computes the remaining circuit using 0 s as the inputs of the honest parties and $\left\{x^{(i)}\right\}_{P_{i} \in T}$ as the inputs of the corrupted parties. For these inputs, it knows the value to be associated with each wire of the circuit. Thus it knows the circuit output $\bar{y}$ resulted from the above set of inputs, namely 0 s as the inputs of the honest parties and $\left\{x^{(i)}\right\}_{P_{i} \in T}$ as the inputs of the corrupted parties.

Start of while loop over the sub-circuits. Set $l=1$ and while $l<L, \mathcal{S}_{f}$ continues as follows:

- Committee Selection. If NewCom $=1$, on receiving $($ sid, $i, \mathcal{L})$ from every party $P_{i} \in T$, $\mathcal{S}_{f}$ on behalf of $\mathcal{F}_{\text {Commitтеe }}$ picks $\mathfrak{c}$ parties from its local set $\mathcal{L}$ at random and assigns them to $\mathcal{C}$. It then sends $\left(\operatorname{sid}, P_{i}, \mathcal{C}\right)$ to every $P_{i} \in T$.

- $[\cdot]$ to $\langle\cdot\rangle_{\mathcal{C}}$ Conversion of Inputs of $\mathrm{ckt}_{l}$. Let $\left[x_{1}\right], \ldots,\left[x_{\mathrm{in}_{l}}\right]$ denote $[\cdot]$-sharing of the inputs to the sub-circuit $\mathrm{ckt}_{l}$. For $k \in\left\{1, \ldots, \operatorname{in}_{l}\right\}, \mathcal{S}_{f}$ invokes the sub-simulator $\mathcal{S}_{[\cdot] \rightarrow\langle\cdot\rangle}$ (Fig. 20) that simulates the steps of the honest parties in $\Pi_{[\cdot] \rightarrow\langle\cdot\rangle}$, with (sid, $\left.\left\{\left[x_{k}\right]_{i}\right\}_{P_{i} \in \mathcal{P} \backslash T}, \mathcal{C}\right)$ (namely with the shares corresponding to the honest parties). The subsimulator returns $\mathcal{S}_{f}$ with $\left(\right.$ sid, $\left.\left\{\left\langle x_{k}\right\rangle_{i}\right\}_{P_{i} \in \mathcal{C} \wedge(\mathcal{P} \backslash T)}\right)$.

- Evaluation of the Sub-circuit $\mathrm{ckt}_{l}$. The simulator $\mathcal{S}_{f}$ invokes the simulator $\mathcal{S}_{\mathrm{ckt}_{l}}^{\mathrm{NR}}$ (namely the simulator of the MPC protocol of [12] with the appropriate modifications in our context to do fault localization) for simulating the steps of the honest parties in the protocol $\Pi_{\mathrm{ckt}_{l}}^{\mathrm{NR}}$.

- $\langle\cdot\rangle_{\mathcal{C}}$ to $[\cdot]$ conversion of Outputs of $\mathrm{ckt}_{l} . \mathcal{S}_{f}$ invokes $\mathcal{S}_{\langle\cdot\rangle \rightarrow[\cdot]}$ with $\left(\right.$ sid, $\left.\left\{\left\langle y_{k}\right\rangle_{i}\right\}_{P_{i} \in \mathcal{C} \wedge(\mathcal{P} \backslash T)}, \mathcal{C}\right)$ for every $k \in$ $\left\{1, \ldots\right.$, out $\left._{l}\right\}$ and gets back either (sid, $\left\{\left[y_{k}\right]_{i}\right\}_{P_{i} \in \mathcal{P} \backslash T}$ ) or (sid, $i$, Failure, $\left.P_{a}, P_{b}\right)$ or (sid, $i$, Failure, $\left.P_{a}\right)$ and does the following:

- If (sid, $\left\{\left[y_{k}\right]_{i}\right\}_{P_{i} \in \mathcal{P} \backslash T}$ ) is received for every $k$, increment $l=l+1$, set NewCom $=0$, store the sharings and return to the while loop.

- If (sid, $i$, Failure, $\left.P_{a}, P_{b}\right)$ is received for some $k \in$ out $_{l}$, update $\mathcal{L}$ as $\mathcal{L}=\mathcal{L} \backslash\left\{P_{a}, P_{b}\right\}, \mathfrak{t}$ as $\mathfrak{t}=\mathfrak{t}-1, \mathfrak{n}$ as $\mathfrak{n}=\mathfrak{n}-2$.

- If (sid, $i$, Failure, $P_{a}$ ) is received for some $k \in$ out $_{l}$, update $\mathcal{L}$ as $\mathcal{L}=\mathcal{L} \backslash\left\{P_{a}\right\}, \mathfrak{t}$ as $\mathfrak{t}=\mathfrak{t}-1, \mathfrak{n}$ as $\mathfrak{n}=\mathfrak{n}-1$.

- Set NewCom $=1$ and go to Committee Selection Step.

Output Rerandomization Let $[\bar{y}]$ denote the [·]-sharing of the output of ckt. $\mathcal{S}_{f}$ invokes $\mathcal{S}_{\text {RandZero[.] }}$ with input $($ sid, $y-\bar{y})$. $\mathcal{S}_{\text {RandZero[.] }}$ simulates the honest parties in protocol $\Pi_{\text {RandZero[.] }}$ and returns to $\mathcal{S}_{f}\left(\right.$ sid, $\left.\left\{[y-\bar{y}]_{i}\right\}_{P_{i} \in(\mathcal{P} \backslash T)}\right) . \mathcal{S}_{f}$ locally computes $[y]_{i}=[\bar{y}]_{i}+[y-\bar{y}]_{i}$ for every $P_{i} \in \mathcal{P} \backslash T$.

Output Computation. On behalf of every honest $P_{i}, \mathcal{S}_{f}$ sends (sid, $i, j, f_{i}, g_{i}, h_{i}$ ) to every $P_{j} \in T$ where $[y]_{i}=$ $\left(f_{i}, g_{i}, h_{i},\left\{\mathbf{C}_{f_{j}, g_{j}, h_{j}}\right\}_{P_{j} \in \mathcal{P}}\right)$. Clearly every $P_{i} \in T$ will recover $y$ at the end due to the output rerandomization step.

The simulator then outputs $\mathcal{A}$ 's output and terminate.

Fig. 19. Simulator for the adversary $\mathcal{A}$ corrupting at most $t$ parties in the set $T \subset \mathcal{P}$ in the protocol $\Pi_{f}$. 
erty of the underlying commitment scheme. For the correctness of the protocol, we rely on the trapdoor security and binding properties of the underlying double trapdoor commitment scheme.

\section{Simulator $\mathcal{S}_{[\cdot] \rightarrow\langle\cdot\rangle}$}

For session id sid, on receiving (sid, $\left\{[s]_{i}\right\}_{P_{i} \in \mathcal{P} \backslash T}, \mathcal{X}$ ) from $\mathcal{S}_{f}, \mathcal{S}_{[\cdot] \rightarrow\langle\cdot\rangle}$ interacts with the corrupted parties in $T$ on behalf of the honest parties in an instance of Protocol $\Pi_{[\cdot] \rightarrow\langle\cdot\rangle}$. The simulator $\mathcal{S}_{[\cdot] \rightarrow\langle\cdot\rangle}$ is aware of the internal state of the honest parties corresponding to the $[s]$. The simulator proceeds as follows:

Verifiably $\langle\cdot\rangle$-sharing the Share and Opening Information in $[s]_{i}$. First, $\mathcal{S}_{[\cdot] \rightarrow\langle\cdot\rangle}$ acts on behalf of every honest $P_{i}$ as the dealer in an instance of $\Pi_{\langle\cdot\rangle}$ with $\left(\right.$ sid, $\left.[s]_{i}, \mathcal{X}\right)$ such that $[s]_{i}=\left(f_{i}, g_{i}, h_{i},\left\{\mathbf{C}_{f_{j}, g_{j}, h_{j}}\right\}_{P_{j} \in \mathcal{P}}\right)$. It also simulates every honest party $P_{i}$ in an instance of $\Pi_{\langle\cdot\rangle}$ where a corrupted $P_{k} \in T$ acts as a dealer.

Identifying the Correctly $\langle\cdot\rangle$-shared Shares of $s$ and Generating $\langle s\rangle_{\mathcal{X}}$. If a corrupted $P_{i} \in T$ is caught cheating during conflict resolution step, then exclude it from a set $\mathcal{H}$ that is initialized to $\mathcal{P}$. Otherwise, for every corrupted $P_{i} \in T$, let it receive $\left\langle f_{i}\right\rangle_{k}$ on behalf of every honest $P_{k} \in(\mathcal{P} \backslash T) \wedge \mathcal{X}$. Without loss of generality, let ${ }^{a} \mathcal{H}=\left\{P_{1}, \ldots, P_{|\mathcal{H}|}\right\}$ and let $c_{1}, \ldots, c_{|\mathcal{H}|}$ be the publicly known Lagrange interpolation coefficients, such that $c_{1} f_{1}+\ldots+c_{|\mathcal{H}|} f_{|\mathcal{H}|}=s$. Then the simulator locally computes $\langle s\rangle_{i}=c_{1}\left\langle f_{1}\right\rangle_{i}+\ldots+c_{|\mathcal{H}|}\left\langle f_{|\mathcal{H}|}\right\rangle_{i}$ on behalf of every honest $P_{i} \in \mathcal{X}$ and returns (sid, $\left.\left\{\langle s\rangle_{i}\right\}_{P_{i} \in \mathcal{X} \wedge(\mathcal{P} \backslash T)}\right)$ to $\mathcal{S}_{f}$.

${ }^{a}$ The set $\mathcal{H}$ will be of size more than $t+1$.

Fig. 20. Simulator $\mathcal{S}_{[\cdot] \rightarrow\langle\cdot\rangle}$ to be Invoked by the MPC Simulator $\mathcal{S}_{f}$ for Simulating the Steps of Sub-protocol $\Pi_{[\cdot] \rightarrow\langle\cdot\rangle}$ in $\Pi_{f}$

\section{Simulator $\mathcal{S}_{\langle\cdot\rangle \rightarrow[\cdot]}$}

For session id sid, on receiving (sid, $\left.\left\{\langle s\rangle_{i}\right\}_{P_{i} \in \mathcal{X} \wedge(\mathcal{P} \backslash T)}, \mathcal{X}\right)$ from $\mathcal{S}_{f}, \mathcal{S}_{\langle\cdot\rangle \rightarrow[\cdot]}$ interacts with the corrupted parties in $T$ on behalf of the honest parties in an instance of Protocol $\Pi_{\langle\cdot\rangle \rightarrow[\cdot]}$. Note that the simulator is aware of the internal state of the honest parties corresponding to the $\langle s\rangle$. The simulator proceeds as follows:

- $\mathcal{S}_{\langle\cdot\rangle \rightarrow[\cdot]}$, on behalf of every honest $P_{i} \in \mathcal{X}$, interprets $\langle s\rangle_{i}$ as $\left(s_{i}, u_{i}, v_{i},\left\{\mathbf{C}_{s_{j}}^{P_{i}, u_{j}, v_{j}}\right\}_{P_{j} \in \mathcal{X}}\right)$ and sends (sid, $k, i,\left\{\mathbf{C}_{s_{j}, u_{j}, v_{j}}^{P_{i}}\right\}_{P_{j} \in \mathcal{X}}$ ) to every $P_{k} \in T$ (acting on behalf of functionality $\mathcal{F}_{\mathrm{BC}}$ that would have been called by $P_{i}$ in the hybrid protocol). On receiving (sid, $i,\left\{{\overline{\mathbf{C}_{s_{j}}^{P_{i}, u_{j}, v_{j}}}}_{\}_{P_{j}} \in \mathcal{X}}, \mathcal{P}\right)$ from every $P_{i} \in T, \mathcal{S}_{\langle\cdot\rangle \rightarrow[\cdot]}$ acts on behalf of $\mathcal{F}_{\mathrm{BC}}$ and sends (sid, $\left.k, i,\left\{\overline{\mathbf{C}_{s_{j}, u_{j}, v_{j}}^{P_{i}}}\right\}_{P_{j} \in \mathcal{X}}\right)$ to every $P_{k} \in T$.

- If there exists a pair of parties $P_{a}, P_{b} \in \mathcal{X}$, such that $P_{a}$ is honest and $P_{b} \in T$ and $\left\{\mathbf{C}_{s_{j}, u_{j}, v_{j}}^{P_{a_{j}}}\right\}_{P_{j} \in \mathcal{X}} \neq$ $\left\{\overline{\mathbf{C}_{s_{j}, u_{j}, v_{j}}^{P_{b}}}\right\}_{P_{j} \in \mathcal{X}}$, then return (sid, Failure, $\left.P_{a}, P_{b}\right)$ to $\mathcal{S}_{f}$ and halt.

- On behalf of every honest $P_{i} \in \mathcal{X}, \mathcal{S}_{\langle\cdot\rangle \rightarrow[\cdot]}$ acts as a D and selects $f^{(i)}(\cdot), g^{(i)}(\cdot)$ and $h^{(i)}(\cdot)$ such that they are random polynomials of degree at most $t$, subject to the condition that $f^{(i)}(0)=s_{i}, g^{(i)}(0)=u_{i}$ and $h^{(i)}(0)=v_{i}$. Then on behalf of $\mathcal{F}_{\mathrm{GeN}[\cdot]}, \mathcal{S}_{\langle\cdot\rangle \rightarrow[\cdot]}$ creates the $\left[s_{i}\right]$ exactly in the way $\mathcal{F}_{\mathrm{GeN}[\cdot]}$ would compute on input $\left(\operatorname{sid}, i, f^{(i)}(\cdot), g^{(i)}(\cdot), h^{(i)}(\cdot)\right)$ from $P_{i}$ as the dealer. Then it hands (sid, $\left.k, i,\left[s_{i}\right]_{k}\right)$ to every $P_{k} \in T$. On receiving $\left(\operatorname{sid}, i, \overline{f^{(i)}}(\cdot), \overline{g^{(i)}}(\cdot), \overline{h^{(i)}}(\cdot)\right)$ from a corrupted $P_{i} \in T$ acting as $\mathrm{D}, \mathcal{S}_{\langle\cdot\rangle \rightarrow[\cdot]}$ acts exactly as $\mathcal{F}_{\mathrm{GEN}[\cdot]}$ and returns either (sid, $i, k$, Failure) or $\left(\right.$ sid, $\left.i, k,\left[s_{k}\right]_{i}\right)$ to every $P_{i} \in T$. $\mathcal{S}_{\langle\cdot\rangle \rightarrow[\cdot]}$ returns (sid, Failure, $P_{k}$ ) to $\mathcal{S}_{f}$ when (sid, $i, k$, Failure) was generated for any $P_{k} \in T$. Otherwise, it locally computes $[s]_{i}=\sum_{P_{k} \in \mathcal{X}}\left[s_{k}\right]_{i}$ for every honest $P_{i} \in \mathcal{P} \backslash T$, returns $\left(\right.$ sid, $\left.\left\{[s]_{i}\right\}_{P_{i} \in \text { Parties } \backslash T}\right)$ to $\mathcal{S}_{f}$ and halts.

Fig. 21. Simulator $\mathcal{S}_{\langle\cdot\rangle \rightarrow[\cdot]}$ to be Invoked by the MPC Simulator $\mathcal{S}_{f}$ for Simulating the Steps of Sub-protocol $\Pi_{\langle\cdot\rangle \rightarrow[\cdot]}$ in $\Pi_{f}$ 


\section{Simulator $\mathcal{S}_{\text {RANDZERO[.] }}$}

For the session id sid, on receiving $(\operatorname{sid}, y-\bar{y})$ from $\mathcal{S}_{f}, \mathcal{S}_{\text {RANDZero[.] }}$ interacts with the corrupted parties in $T$ on behalf of the honest parties in an instance of Protocol $\Pi_{\text {RANDZERo[.] }}$. The simulator proceeds as follows:

\section{Publicly Committing 0:}

- On behalf of honest party $P_{h}$ (it just chooses any honest party from the set $\mathcal{P}$ ) randomly selects $u_{h}, v_{h} \in \mathbb{F}_{p}$, sets $r_{h}=y-\bar{y}$ and computes $\mathbf{C}_{r_{h}, u_{i}, v_{i}}=\operatorname{Comm}_{\mathrm{ck}}\left(y-\bar{y} ; u_{i}, v_{i}\right)$. On behalf of every other honest party $P_{i}$, it randomly selects $u_{i}, v_{i} \in \mathbb{F}_{p}$, sets $r_{i}=0$ and computes $\mathbf{C}_{r_{i}, u_{i}, v_{i}}=\operatorname{Comm}_{\mathrm{ck}}\left(r_{i} ; u_{i}, v_{i}\right)$. On behalf of $\mathcal{F}_{\text {ZK.BC corresponding }}$ to every honest $P_{i}$, it then sends (sid, $\left.i, \mathbf{C}_{r_{i}, u_{i}, v_{i}}\right)$ to every $P_{j} \in T$. On receiving (sid, $\left.i, \mathbf{C}_{r_{i}, u_{i}, v_{i}}, u_{i}, v_{i}\right)$ from every corrupted $P_{i} \in T$, it acts as $\mathcal{F}_{\mathrm{ZK} . \mathrm{BC}}$ and verifies if $\mathbf{C}_{r_{i}, u_{i}, v_{i}}=\operatorname{Comm}_{\mathrm{ck}}\left(0 ; u_{i}, v_{i}\right)$. It the tests passes, then the simulator on behalf of $\mathcal{F}_{\mathrm{ZK} . \mathrm{BC}}$ sends (sid, $\left.i, \mathbf{C}_{r_{i}, u_{i}, v_{i}}\right)$ to every $P_{j} \in T$. Otherwise, it sends (sid, $i, \perp$ ) to every $P_{j} \in T$.

- It then constructs a set $\mathcal{T}$, initialized to $\emptyset$ and include in $\mathcal{T}$ all the honest parties in $\mathcal{P}$ and $P_{i} \in T$ if $\mathbf{C}_{r_{i}, u_{i}, v_{i}}=$ $\operatorname{Comm}_{\mathrm{ck}}\left(0 ; u_{i}, v_{i}\right)$ was true for $P_{i}$.

$[\cdot]$-sharing 0 :

- On behalf of honest party $P_{i}$, it selects three random polynomials $f^{(i)}(\cdot), g^{(i)}(\cdot)$ and $h^{(i)}(\cdot)$ each of degree at most $t$, subject to the condition that $f^{(i)}(0)=r_{i}, g^{(i)}(0)=u_{i}$ and $h^{(i)}(0)=v_{i}$. On behalf of $\mathcal{F}_{\mathrm{GEN}[\cdot]}$ for an honest $P_{i}$, it sends (sid, $\left.j, i, f_{j}^{(i)}, g_{j}^{(i)}, h_{j}^{(i)}\right)$ to every $P_{j} \in T$. Then for every corrupted $P_{i} \in T$, on receiving (sid, $\left.i, f^{(i)}(\cdot), g^{(i)}(\cdot), h^{(i)}(\cdot)\right)$ from $P_{i}$ (as the dealer), $\mathcal{S}_{\text {RandZero [. }]}$, on behalf of $\mathcal{F}_{\text {Gen [.] }}$, sends $\left(\operatorname{sid}, j, i,\left[f^{(i)}(0)\right]_{i}\right)$ to every $P_{j} \in T$, after verifying the polynomials $f^{(i)}(\cdot), g^{(i)}(\cdot), h^{(i)}(\cdot)$ with respect to the corresponding commitment $\mathbf{C}_{r_{i}, u_{i}, v_{i}}$ (as done by the functionality $\mathcal{F}_{\mathrm{GeN}[\cdot]}$ ). If the polynomials fails the test, remove $P_{i}$ from $\mathcal{T}$.

- It locally computes $[y-\bar{y}]_{i}=\sum_{P_{j} \in \mathcal{T}}\left[r_{j}\right]_{i}$ and returns $\left(\right.$ sid, $\left.\left\{[y-\bar{y}]_{i}\right\}_{P_{i} \in \mathcal{P} \backslash T}\right)$ to $\mathcal{S}_{f}$ and halt.

Fig. 22. Simulator for $\Pi_{\mathrm{RANDZ} \text { Ro[·] }}$ 